\title{
ON THE NIWA-SHINTANI THETA-KERNEL LIFTING OF MODULAR FORMS
}

\author{
BARRY A. CIPRA
}

Modular forms of half-integral weight are of intrinsic interest: many of the functions of classical number theory transform under a matrix group with half-integral weight. The aim of this paper is to refine some results and techniques which have been introduced to study these functions and the arithmetic information which they contain.

Our results will be most clear if we give a very brief history of the subject. The general theory of modular forms of half-integral weight is a fairly recent development. Although Hecke [4] did some work in the area, in a sense the subject really begins with Shimura's 1973 paper, "On modular forms of half-integral weight" [11].

Shimura demonstrated an extraordinary 'lifting' property for modular forms of half-integral weight. By considering Euler products associated to eigenfunctions of Hecke operators, Shimura constructs a family of maps taking cusp forms of half-integral weight to holomorphic forms of even, integral weight, which is where the subject has been most studied and best understood. This 'lifting', moreover, takes eigenfunctions to eigenfunctions.

While Shimura proves the lifted functions to be modular forms, he does not completely determine the level at which they transform. However, he makes the following conjecture: if the original function transforms at level $4 N$, then the lifted form transforms at level $2 N$.

Shimura also proves that the lifted forms are in fact cusp forms, if the half-integral weight is $\geq 5 / 2$. The remaining case, weight $3 / 2$ (weight $1 / 2$ does not come under consideration), is more complicated. Certain forms, namely the 'theta functions', fail to lift to cusp forms. Shimura here conjectures that everything in the 'orthogonal complement' (with respect to the Petersson inner product) does lift to a cusp form.

Received December 25, 1981. 
The first conjecture was largely answered by Niwa [8], using important techniques introduced by Shintani [12]. Niwa established that, for weight $\geq 7 / 2$, Shimura's lifting can be obtained by taking the Petersson inner product of the original cusp form against a suitably constructed 'theta kernel' of two variables. Under this construction, the correct transformation at level $2 N$ is easily seen.

The second conjecture, concerning weight $3 / 2$, has been affirmed by various researchers, including the author in his Ph.D. dissertation (which this paper is based upon). The techniques have been different: Flicker [2] and Gelbart and Piatetski-Shapiro [3] have used the machinery of representation theory; Kojima [7] gives a proof in the context of Dirichlet series; this author gives a direct proof. It turns out that the behavior of a lifted form at cusps is determined by the original cusp form's inner product against theta functions (Theorems 4.4 and 4.9).

Our goal in this paper is two-fold: to widen the applicability of Niwa's theta kernel to include the cases of weight $5 / 2$ and $3 / 2$, and then to use these results to give a direct proof of Shimura's second conjecture. Our actual results are more general. We find that Niwa's theta kernel applies (with one exception) to all holomorphic modular forms of all positive, half-integral weights, including theta functions of weight $1 / 2$. (The exception is $\theta(z)$ itself!) Our proof of Shimura's conjecture actually does two cases: it prescribes values at cusps for lifts from weight $3 / 2$, and poleterms at cusps for lifts from weight $1 / 2$. These values and pole-terms are equated by explicit formulae to the inner product of the initial form against appropriate theta functions of its weight.

The structure of the paper is as follows:

In Section 1, we consider a general theory of theta kernels for $S L$ $(2, Z)$, using Shintani's presentation of the Weil representation. The critical ideas are that of a function $f$ with two 'spherical' properties, and a function $\omega$ with two 'permutation' properties. We prove some important growth estimates for theta kernels (Corollary 1.13). We illustrate the theory with the simple case of a quadratic form in one variable: this gives rise to the classical theta functions, and to some other technical results which are needed later.

In Section 2, we introduce the particular theta kernel used by Niwa to reproduce Shimura's lifting. We also 'invert' this theta kernel, which makes this formulation of the lifting more direct. We prove four main 
results: 1) The theta kernel is (with one exception) 'rapidly decreasing' at all cusps, so that its inner product is well-defined against any 'slowly growing' modular form (Proposition 2.8-this uses the growth estimates of Section 1). 2) When the second (integral-weight) variable is restricted to its imaginary axis, the theta kernel can be re-expressed as a 'Poincaré' series-i.e., as a sum over coset representatives (Theorem 2.11). 3) The inner-product lifting agrees with Shimura's lifting, at least on the imaginary axis (Theorem 2.12). 4) The inner-product lifting is holomorphic (and thus agrees with Shimura's lifting everywhere-Theorems 2.16 and 2.17).

The key issue is holomorphy. The definition of the inner product looks hopelessly non-holomorphic, and indeed the theta-kernel is not holomorphic in either variable. Holomorphy of the inner product depends on three ingredients: a differential equation (2.52) satisfied by the inner product, periodicity of the inner product (so that we can begin separating out solutions of the differential equation into holomorphic and non-holomorphic Fourier expansions), and certain growth estimates which the nonholomorphic piece (were there any!) is certain to violate. In Niwa, simple estimates suffice for weights $\geq 7 / 2$. These however fail at the lowest weights. Our proof accounts for all weights by bringing in subtler estimates. These are based on the aforementioned result that, on the imaginary axis, Niwa's inner product reproduces Shimura's lift-for which the behavior at infinity is clear. (To be precise, we should note a discrepancy at weight $1 / 2$ : The lifts here are not holomorphic; rather they are equal parts holomorphic and anti-holomorphic.)

Section 3 specifies the liftings of the theta functions of weights $1 / 2$ and 3/2 and derives a formula (Theorem 3.4) for their behavior at cusps.

In Section 4, we prove Shimura's conjecture for weight $3 / 2$ and an analogous result for weight $1 / 2$. The proof treats both results simultaneously, but comes in two parts: a special case $(t=1)$ and the general case (arbitrary, square-free $t$, where the proof amounts to reducing to the special case). In the beginning of the proof, we also see why these results occur only for weights $3 / 2$ and $1 / 2$. Essentially what happens is this: as one approaches a cusp of the lifted form, the theta kernel degenerates into a linear combination of theta functions. Thus if the original cusp form (of weight $3 / 2$ ) is orthogonal to all the appropriate theta functions, then its lifting will be a cusp form. The converse is easily seen to be true also. 
The main results of the section are contained in Theorems 4.4 and 4.9, where the value or pole term of the lifted form at a cusp is given explicitly as an inner product of the original form against a linear combination of theta functions.

Finally, in Section 5, we give some examples. We derive a classical formula for the number of representations of a (square-free) number as the sum of three squares; we identify two modular forms of weight $3 / 2$ and low level; and we derive formulas for the Petersson 'norm' of some theta functions. These examples have proved useful chiefly for checking the accuracy of our main formulas.

I would like to thank my advisors Mike Razar and Steve Kudla of The University of Maryland, for introducing me to this subject and for their generous donation of time and knowledge. I would also like to thank Prof. John Benedetto for his timely encouragement.

\section{Notations}

We follow the notation established by Shimura, Niwa, and Shintani $[11,8,12]$. As usual, $Z, \boldsymbol{R}$, and $\boldsymbol{C}$ denote the integers, reals, and complex numbers. We write $q=e(z)=\exp (2 \pi i z)$ where, typically, $z=u+i v \in H$ $=\{z \mid \operatorname{Im} z>0\}$. We will also need a second complex variable, which we denote by $w=\xi+i \eta$. When we take square roots, we make a cut along the negative real axis and take the 'positive' square root.

In general, we denote an arbitrary matrix in $S L(2, R)$ by $\sigma=\left(\begin{array}{ll}a & b \\ c & d\end{array}\right)$, while $\gamma=\left(\begin{array}{ll}a & b \\ c & d\end{array}\right)$ denotes an arbitrary matrix in $S L(2, Z)$. Our most important group is $\Gamma_{0}(N)=\{\gamma \mid c \equiv 0 \bmod N\}$, where $N$ is a positive integer. We also use the subgroup $\Gamma_{\infty}=\{\gamma \mid c=0\}$.

If $k \in Z, \sigma \in G L^{+}(2, R)$, and $f$ is a function on $H$, define

$$
\left(\left.f\right|_{k} \sigma\right)(z)=(\operatorname{det} \sigma)^{k / 2}(c z+d)^{-k} f(\sigma z) .
$$

When $k \in Z$ is odd, and $\gamma \in \Gamma_{0}(4 N)$, define

$$
\left(\left.f\right|_{\kappa / 2} \gamma\right)(z)=j(\gamma, z)^{-\kappa} f(\gamma z)
$$

where

$$
j(\gamma, z)=\theta(\gamma z) / \theta(z)=\varepsilon_{d}^{-1}\left(\frac{c}{d}\right)(c z+d)^{1 / 2} .
$$

Here $\theta(z)=\sum_{-\infty}^{\infty} e\left(n^{2} z\right), \varepsilon_{d}=1$ or $i$ as $d \equiv 1$ or $3 \bmod 4$, and $(c / d)$ is the quadratic residue symbol as defined in Shimura [11, p. 442-see also Ap- 
pendix A of this paper]. We define also the 'inversion' operator $W(N)$ following Serre-Stark [10]:

$$
\left.f\right|_{k}(W(N))(z)= \begin{cases}N^{-k / 2}(-i z)^{-k} f(-1 / N z) & k=\text { half-integer } \\ N^{-k / 2} z^{-k} f(-1 / N z) & k=\text { even-integer }\end{cases}
$$

Let $k$ be an integer or half-integer, and let $N$ be a positive integer, divisible by 4 if $k$ is a half-integer. Let $\chi$ be a character $\bmod N$. We say that a function $f$ is a modular form of weight $k$, level $N$, and character $\chi$-and write $f \in M_{k}(N, \chi)$-if and only if $\left.f\right|_{k} \gamma=\chi(d) f$ for all $\gamma \in \Gamma_{0}(N)$. Since $\left(\begin{array}{rr}-1 & 0 \\ 0 & -1\end{array}\right) \in \Gamma_{0}(N)$, we must assume $\chi$ to be even for $k$ half- or evenintegral, and $\chi$ odd for $k$ odd-otherwise the space $M_{k}$ is trivial.

If $f \in M_{k}(N, \chi)$ is holomorphic on $H$ and has a Fourier expansion at every cusp (see [11]), we call $f$ a holomorphic modular form and write $f$ $\in G_{k}(N, \chi)$. If such an $f$ vanishes at every cusp (i.e., the constant term in every Fourier expansion is zero, so that $f$ is 'rapidly decreasing' at cusps), we call $f$ a cusp form, and write $f \in S_{k}(N, \chi)$.

If $f, g \in M_{k}(N, \chi)$, we define the Petersson inner product

$$
\langle f, g\rangle=\int_{\Gamma_{0}(N) \backslash H} v^{k} f(z) \overline{g(z)} d_{0} z
$$

whenever the integral is well-defined (i.e., absolutely convergent). Here $d_{0} z$ denotes the invariant area element $d u d v / v^{2}$.

Lastly, we shall have occasion to use Hermite polynomials, so let us define the ones we will use. For $0 \leq \nu \in Z$, define

$$
H_{\nu}(x)=(-1)^{\nu} \exp \left(x^{2} / 2\right) \frac{d^{\nu}}{d x^{\nu}} \exp \left(-x^{2} / 2\right) \text {. }
$$

Thus $H_{0}(x)=1, H_{1}(x)=x$, and so forth.

\section{$\S 1$. General theta kernels}

We begin by summarizing Shintani's presentation of the Weil representation.

(1) Let $Q$ be a rational symmetric matrix of signature $(p, q), p+q$ $=n$. For $x, y \in \boldsymbol{R}^{n}$, define the inner product

$$
\langle x, y\rangle={ }^{t} x Q y .
$$

For matrices $\sigma=\left(\begin{array}{ll}a & b \\ c & d\end{array}\right) \in S L(2, R)$ and Schwartz functions $f \in \mathscr{S}\left(\boldsymbol{R}^{n}\right)$, we define the Weil representation 


$$
(r(\sigma, Q) f)(x)=\left\{\begin{array}{l}
|a|^{n / 2} e\left[\frac{a b}{2}\langle x, x\rangle\right] f(a x) \quad \text { if } c=0 \\
|\operatorname{det} Q|^{-1 / 2}|c|^{-n / 2} \int_{R^{n}} e\left[\frac{a\langle x, x\rangle-2\langle x, y\rangle+d\langle y, y\rangle}{2 c}\right] f(y) d y
\end{array}\right.
$$

Since this is just a mixture of the Fourier transform, scaling, and multiplication by functions of modulus one, there is an extension to functions $f \in L^{2}\left(\boldsymbol{R}^{n}\right)$. The Weil representation is a projective unitary representation of $S L(2, R)$ in $L^{2}\left(R^{n}\right)$, in that

$$
r(\sigma \tau, Q)=c(\sigma, \tau) r(\sigma, Q) r(\tau, Q)
$$

with $|c(\sigma, \tau)|=1$. More precisely, defining

and

$$
J(\sigma, z)=c z+d
$$

we have

$$
\varepsilon(\sigma)= \begin{cases}\sqrt{i} & c>0 \\ i^{(1-\operatorname{sgn} d) / 2} & c=0 \\ \sqrt{i}^{-1} & c<0\end{cases}
$$

where

$$
c(\sigma, \tau)=\{\varepsilon(\sigma \tau) / \varepsilon(\sigma) \varepsilon(\tau)\}^{p-q} c_{0}(\sigma, \tau)^{q-p}
$$

$$
c_{0}(\sigma, \tau)=\sqrt{J(\sigma \tau, i)} / \sqrt{J(\sigma, \tau i)} \sqrt{J(\tau, i)} .
$$

The Weil representation becomes a true (not projective) representation if we pass to the two-fold covering of $S L(2, R)$, the metaplectic group: Let

$$
\widehat{S L}(2, R)=\left\{(\sigma, t) \mid \sigma \in S L(2, R), \quad t= \pm 1 ; \quad(\sigma, t)\left(\sigma^{\prime}, t^{\prime}\right)=\left(\sigma \sigma^{\prime}, t t^{\prime} c_{0}\left(\sigma, \sigma^{\prime}\right)\right)\right\} .
$$

Then

$$
r_{0}((\sigma, t), Q) f=t^{p-q} \varepsilon(\sigma)^{q-p} r(\sigma, Q) f
$$

is a representation of $\widehat{S L}(2, R)$ in $L^{2}\left(\boldsymbol{R}^{n}\right)$.

(2) Let $L$ be a lattice in $R^{n}$ such that $\langle x, x\rangle \in 2 Z$ for all $x \in L$. (Thus for $x, y \in L,\langle x, y\rangle=\frac{1}{2}(\langle x+y, x+y\rangle-\langle x, x\rangle-\langle y, y\rangle) \in Z$.) Let $L^{*}$ be the dual lattice: $L^{*}=\left\{x \in \boldsymbol{R}^{n} \mid\langle x, y\rangle \in Z\right.$ for all $\left.y \in L\right\}$. Clearly $L \subset L^{*}$, and $L^{*} / L$ is a finite abelian group. Denote by $v(L)$ the volume of a fundamental parallelotope of $L$ in $R^{n}$ :

$$
v(L)=\int_{R^{n / L}} d x
$$


Let $f \in \mathscr{S}\left(\boldsymbol{R}^{n}\right)$ and define, for $h \in L^{*} / L$,

$$
\theta(f, h)=\sum_{x \in L} f(x+h) .
$$

We quote our first result from Shintani [12, p. 95]:

Proposition 1.1. Let $\gamma=\left(\begin{array}{ll}a & b \\ c & d\end{array}\right) \in S L(2, Z)$. Then

$$
\theta(r(\gamma, Q) f, h)=\sum_{k \in L^{*} / L} c(h, k)_{\gamma} \theta(f, k)
$$

where

$$
c(h, k)_{r}=\left\{\begin{array}{cl}
\delta_{h, a k} e\left(\frac{a b}{2}\langle h, k\rangle\right) \quad \text { if } c=0 \\
|\operatorname{det} Q|^{1 / 2} v(L)^{-1}|c|^{-n / 2} \sum_{r \in L / c L} e\left[\frac{1}{2 c}(a<h+r, h+r\rangle-2\langle k, h+r\rangle\right. \\
+d\langle k, k\rangle] \quad \text { if } c \neq 0
\end{array}\right.
$$

(ii) Assume c to be even and nonzero, $c L^{*} \subset L$, and $c\langle x, x\rangle$ to be even for all $x \in L^{*}$. Let $\left\{\lambda_{1}, \cdots \lambda_{n}\right\}$ be a $Z$-basis for $L$, and define $D=\operatorname{det}\left(\left\langle\lambda_{i}\right.\right.$, $\left.\lambda_{j}\right\rangle$ ). Then

$$
c(h, k)_{r}=\delta_{h, d k} \sqrt{i}^{(p-q) \operatorname{sgn}(c d)} e\left[\frac{a b}{2}\langle h, h\rangle\right] \begin{cases}\varepsilon_{d}^{n}\left(\frac{-2 c}{d}\right)^{n}\left(\frac{D}{d}\right) \quad d>0 \\ \varepsilon_{d}^{-n}(i \operatorname{sgn} c)^{n}\left(\frac{2 c}{d}\right)^{n}\left(\frac{D}{-d}\right) \quad d<0\end{cases}
$$

Remarks. 1. $D$ depends only on $L$ (and $Q$ ), not on the choice of basis. 2. Since $\varepsilon_{d} \varepsilon_{-d}=i$, the actual difference between $d>0$ and $d<0$ in (1.5) is the presence or absence of the term $(-1 / d)^{n}$; otherwise both are $\varepsilon_{|d|}^{n}(2 c /|d|)^{n}$ $(D /|d|)$.

The group generated by those matrices satisfying the hypotheses of (ii) above clearly form a congruence subgroup of the form $\Gamma_{0}(4 N)$, for some positive integer $N$. In our use of it, $\Gamma_{0}(4 N)$ will always satisfy those hypotheses.

For $\left(\begin{array}{ll}a & b \\ c & d\end{array}\right) \in \Gamma_{0}(4 N)$, the map $k \rightarrow d k$ is (among other things) a permutation of $L^{*} / L$. Now suppose $\omega: L^{*} / L \rightarrow C$ is a function satisfying

1) $\omega(k)=0$ if $\langle k, k\rangle \notin 2 Z$

2) $\omega(d k)=\chi(d) \omega(k)$ for $\gamma=\left(\begin{array}{ll}a & b \\ c & d\end{array}\right) \in \Gamma_{0}(4 N)$, 
where $\chi$ is a character $\bmod 4 N$. We say that $\omega$ has the 'first permutation property' for $\Gamma_{0}(4 N)$ with character $\chi$. (It is easy to create such functions: if $\nu: L^{*} / L \rightarrow C$ is any function, nonzero only for $k$ such that $\langle k, k\rangle \in 2 Z$, let

$$
\left.\omega(k)=\frac{1}{\phi(4 N)} \sum_{d=1}^{4 N} \bar{\chi}(d) \nu(d k) .\right)
$$

As an immediate consequence, we have

THEOREM 1.2. Suppose $\omega$ has the first permutation property for $\Gamma_{0}(4 N)$ and $\chi$. Define

$$
\theta(f, \omega)=\sum_{h \in L^{*} / L} \omega(h) \theta(f, h)=\sum_{k \in L^{*}} \omega(k) f(k) .
$$

Then, for $\gamma=\left(\begin{array}{ll}a & b \\ c & d\end{array}\right) \in \Gamma_{0}(4 N)$

$$
\theta(r(\gamma, Q) f, \omega)=\sqrt{i}^{(p-q) \operatorname{sgn}(c d)}\left\{\begin{array}{c}
\varepsilon_{d}^{n}\left(\frac{-2 c}{d}\right)^{n}\left(\frac{D}{d}\right) \\
\varepsilon_{d}^{-n}(i \operatorname{sgn} c)^{n}\left(\frac{2 c}{d}\right)\left(\frac{D}{-d}\right)
\end{array}\right\} \chi(d) \theta(f, \omega)
$$

taking the top (bottom) choice as $d>0(d<0)$.

(3) $S L(2, \boldsymbol{R})$ is a locally compact group, so it carries a Haar measure $d g$. There are various ways of expressing $d g$. If $g=\left(\begin{array}{ll}x_{1} & x_{2} \\ x_{3} & x_{4}\end{array}\right)$, we can set $d g=d x_{2} d x_{3} d x_{4} /\left|x_{4}\right|$. Writing $g=\left(\begin{array}{ll}a & 0 \\ 0 & a^{-1}\end{array}\right)\left(\begin{array}{ll}1 & x \\ 0 & 1\end{array}\right)\left(\begin{array}{rr}\cos \phi & \sin \phi \\ -\sin \phi & \cos \phi\end{array}\right)$, we have $d g=d a d x d \phi /|a| . \quad$ If $g=\left(\begin{array}{ll}v^{1 / 2} & u v^{-1 / 2} \\ 0 & v^{-1 / 2}\end{array}\right)\left(\begin{array}{rr}\cos \phi & \sin \phi \\ -\sin \phi & \cos \phi\end{array}\right)$, then $d g=\left(d u d u / v^{2}\right)$ $d \phi$. This last expression leads us to identify the upper half-plane $H=$ $\{u+i v \mid v>0\}$ with $S L(2, R) / K$, where

by the map

$$
K=\left\{k(\phi)=\left(\begin{array}{rr}
\cos \phi & \sin \phi \\
-\sin \phi & \cos \phi
\end{array}\right) \mid-\pi<\theta \leq \pi\right\},
$$

$$
g K \rightarrow z=g i
$$

This is well defined, since $k i=i$ for $k \in K$. Under this map $\left(\begin{array}{ll}v^{1 / 2} & u v^{-1 / 2} \\ 0 & v^{-1 / 2}\end{array}\right)$ goes to $u+i v$; we call this matrix $\sigma_{z}$.

Proposition 1.3. Let $\tau \in S L(2, R)$, and $\sigma_{z}=\left(\begin{array}{ll}v^{1 / 2} & u v^{-1 / 2} \\ 0 & v^{-1 / 2}\end{array}\right)$ for $z=u+$ $i v \in H$. Define $\phi(\bmod 2 \pi)$ by $e^{-i \phi}=J(\tau, z) /|J(\tau, z)|$, and let

$$
k(\phi)=\left(\begin{array}{rr}
\cos \phi & \sin \phi \\
-\sin \phi & \cos \phi
\end{array}\right) \text { Then }
$$


1) $\tau \sigma_{z}=\sigma_{\tau z} k(\phi)$

2) $r(\tau, Q) r\left(\sigma_{z}, Q\right)=r\left(\sigma_{z z}, Q\right) r(k(\phi), Q)$

Proof. As actions on $H$, both sides of 1 ) take $i$ to $\tau z$. Therefore the two sides differ only by a 'rotation' matrix. Observe that both sides also take the cusp at $\cot \phi$ to the cusp at $i \infty$ : for the RHS this is clear, while for the LHS it follows since, from the definition of $\theta, \cot \theta=-(c u+d) / v$. Therefore the two sides differ only by $\pm I$. Equality now follows by continuity and connectedness, since $I \cdot \sigma_{z}=\sigma_{I z} k(0)$ is obvious.

Statement 2) now follows by comparing $c(\sigma, \tau)$ for the matrices on each side.

Corollary 1.4. For $\tau=\left(\begin{array}{ll}a & b \\ c & d\end{array}\right) \in S L(2, R)$, and $t \in R$, let $\tau_{t}=\left(\begin{array}{ll}a & b t^{2} \\ c / t^{2} & d\end{array}\right)$ $=\left(\begin{array}{ll}t & \\ & t^{-1}\end{array}\right) \tau\left(\begin{array}{cc}t^{-1} & \\ & t\end{array}\right)$. Let $k(\phi)$ be as before. Then

$$
\tau_{t} \sigma_{t^{2} z}=\sigma_{t^{2}(\tau z)} k(\phi)
$$

and

$$
r\left(\tau_{t}, Q\right) r\left(\sigma_{t^{2} z}, Q\right)=r\left(\sigma_{t^{2}(\tau z)}, Q\right) r(k(\phi), Q) .
$$

Proof. This follows since $J\left(\tau_{t}, t^{2} z\right) /\left|J\left(\tau_{t}, t^{2} z\right)\right|=J(\tau, z) /|J(\tau, z)|$.

(4) Let $f \in \mathscr{S}\left(\boldsymbol{R}^{n}\right)$ and let $\kappa \in Z$. Assume that for all

$$
\begin{gathered}
k(\phi)=\left(\begin{array}{rr}
\cos \phi & \sin \phi \\
-\sin \phi & \cos \phi
\end{array}\right), \\
r(k(\phi), Q) f=\varepsilon(k(\phi))^{p-q} \sqrt{e^{-i \bar{\phi}-\varepsilon} f}
\end{gathered}
$$

(with $\varepsilon$ defined by (1.2)). We say that such a function $f$ has the 'first spherical property' for weight $\kappa / 2$.

Remarks. 1) By comparing both sides of (1.7) for $\phi=\pi$, it is easy to see that $f$ is identically zero unless $\kappa \equiv n \bmod 2$. 2) The condition (1.7) is really stating that $f$ be an eigen-function for $K \times\{ \pm 1\}$, a maximal compact subgroup of $\widehat{S L}(2, R)$, under the Weil representation $r_{0}$. The characters of this subgroup are of the form $\chi_{m}((k(\phi), t))=t^{m} \sqrt{e^{-i \phi}-m}$, for $m \in Z$. Thus if $f$ has the first spherical property, and $\kappa \equiv n \bmod 2$, we have

$$
r_{0}((k(\phi), t), Q) f=t^{p-q} \varepsilon(k(\phi))^{q-p} r(k(\phi), Q) f=t^{x} \sqrt{e^{-i \phi}-x} f .
$$

Let $f$ have the first spherical property for weight $\kappa / 2$, and let $\omega$ have the first permutation property for $\Gamma_{0}(4 N)$ with character $\chi$. Define 


$$
\theta(z, f, h)=v^{-\kappa / 4} \theta\left(r\left(\sigma_{z}, Q\right) f, h\right) \quad h \in L^{*} / L
$$

and

$$
\theta(z, f, \omega)=\sum_{h \in L^{*} / L} \omega(h) \theta(z, f, h) .
$$

Theorem 1.5. Let $\gamma=\left(\begin{array}{ll}a & b \\ c & d\end{array}\right) \in S L(2, Z)$. Then

$$
(c z+d)^{-\kappa / 2} \theta(\gamma z, f, h)=\sqrt{i}-(p-q) \operatorname{sgn} c \sum_{k \in L^{*} / L} c(h, k)_{\gamma} \theta(z, f, k)
$$

with $c(h, k)_{r}$ as in Proposition 1.1 .

Proof. This follows easily from Propositions 1.1 and 1.3, and the standard fact that $\operatorname{Im}(\gamma z)=\operatorname{Im}(z) /|c z+d|^{2}$.

Corollary 1.6. Let $\gamma=\left(\begin{array}{ll}a & b \\ c & d\end{array}\right) \in \Gamma_{0}(4 N)$. Then

$$
j(\gamma, z)^{-\kappa} \theta(\gamma z, f, \omega)=\chi^{\prime}(d) \theta(z, f, \omega)
$$

where

$$
\chi^{\prime}(d)=\left(\frac{-1}{d}\right)^{(x-n) / 2}\left(\frac{2}{d}\right)^{n}\left(\frac{D}{d}\right)\left((-1)^{q} D, d\right)_{\infty} \chi(d)
$$

with the Hilbert symbol $(x, y)_{\infty}=\left\{\begin{aligned}-1 & \text { if } x, y<0 \\ 1 & \text { otherwise }\end{aligned}\right.$.

CoRollary 1.7. As special cases of Theorem 1.5 we have

(1) $\theta(-1 / z, f, h)=\left.\sqrt{i}^{-(p-q)}{z^{k / 2}}^{\prime} \operatorname{det} Q\right|^{-1 / 2} v(L)^{-1} \sum_{k \in L^{*} / L} e(-\langle k, h\rangle) \theta(z, f, k)$

(2) $\theta(z+1, f, h)=e\left(\frac{1}{2}\langle h, h\rangle\right) \theta(z, f, h)$.

Corollary 1.8. Let $\gamma \in S L(2, Z)$. Then

$$
\left.v^{(x-n) / 4}(c z+d)^{-\kappa / 2} \theta(\gamma z, f, h)\right|_{z=i \infty}=\sqrt{i^{-(p-q) \operatorname{sgn}(c)}} c(h, 0)_{\gamma} f(0)
$$

Assume moreover that $f(0)=0$. Then $\theta(z, f, h)$ is 'rapidly decreasing' at all cusps. That is, for all $\gamma \in S L(2, Z)$, and all $m \geq 0,\left|v^{m}(c z+d)^{-\kappa / 2} \theta(\gamma z, f, h)\right|$ $\rightarrow 0$ as $v \rightarrow \infty$.

Proof. The first equation is easily seen from Theorem 1.5. We postpone the proof of the second assertion to Corollary 1.13, where we give a sharper version of the result.

(5) Which functions-if any-have the first spherical property? It suffices to answer the question when $Q$ is a diagonal matrix. 
Theorem 1.9. Assume $\langle x, y\rangle=m_{1} x_{1} y_{1}+\cdots+m_{p} x_{p} y_{p}-m_{p+1} x_{p+1} y_{p+1}$ $-\cdots-m_{p+q} x_{p+q} y_{p+q}$, with $m_{j}>0$ for all $j$. Let $\nu=\left(\nu_{1}, \cdots, \nu_{p+q}\right)$ be an n-tuple of nonnegative integers such that

$$
(p-q)+2\left(\nu_{1}+\cdots+\nu_{p}-\nu_{p+1}-\nu_{p+q}\right)=\kappa
$$

and define

$$
f_{\nu}=\prod_{j=1}^{p+q} H_{\nu j}\left(2 \sqrt{\pi m_{j}} x_{j}\right) \exp \left(-\pi m_{j} x_{j}^{2}\right)
$$

(The Hermite polynomials $H_{n}$ are defined in the Notations.) Then $f_{\nu}$ has the first spherical property for weight $\kappa / 2$. Conversely, any function with the first spherical property for weight $\kappa / 2$ is a sum (possibly infinite)

$$
f=\sum_{\nu} c_{\nu} f_{\nu}
$$

over all $n$-tuples $\nu$ satisfying (1.14).

Proof. Modifying Niwa's argument [8, p. 150-151], we define the integral transform

where

$$
I(f)(z)=\int_{R^{n}} f(x) k(x, z) d x \quad z=\left(z_{1}, \cdots, z_{n}\right) \in C^{n}
$$

$$
k(x, z)=\exp \left(-\pi \sum_{j=1}^{p+q} m_{j}\left(x_{j}^{2}+2 i x_{j} z_{j}-\frac{1}{2} z_{j}^{2}\right)\right) .
$$

Following definitions, we see

$$
\begin{aligned}
I(r(k(\phi), Q) f)(z)=|\operatorname{det} Q|^{1 / 2}|\sin \phi|^{-n / 2} \int_{R^{n}} \int_{R^{n}} \exp \left[-\pi \sum_{j=1}^{p+q} m_{j}\left\{\left(1 \pm i \frac{\cos \phi}{\sin \phi}\right) x_{j}^{2}\right.\right. \\
\left.\left.\quad+2 i\left(z_{j} \mp y_{j} / \sin \phi\right) x_{j}-\left(\frac{1}{2} z_{j}^{2} \mp i y_{j}^{2} \cos \phi / \sin \phi\right)\right\}\right] f(y) d y d x \\
=|\operatorname{det} Q|^{1 / 2}|\sin \phi|^{-n / 2} \int_{R^{n}} \prod_{j=1}^{p+q} \int_{-\infty}^{\infty} \exp \left[-\pi m_{j}\left(1 \pm i \frac{\cos \phi}{\sin \phi}\right) x_{j}^{2}\right. \\
\left.\quad-2 \pi i m_{j}\left(z_{j} \mp y_{j} / \sin \phi\right) x_{j}+\pi m_{j}\left(\frac{1}{2} z_{j}^{2} \mp i y_{j}^{2} \cos \phi / \sin \phi\right)\right] d x_{j} f(y) d y \\
=|\operatorname{det} Q|^{1 / 2}|\sin \phi|^{-n / 2} \int_{R^{n}} \prod_{j=1}^{p+q}\left(\mp i \frac{\sin \phi}{m_{j}} e^{ \pm i \phi}\right)^{1 / 2} \\
\quad \times \exp \left[-\pi m_{j}\left(y_{j}^{2}+2 i y_{j} e^{ \pm i \phi} z_{j}-\frac{1}{2} e^{ \pm 2 i \phi} z_{j}^{2}\right)\right] f(y) d y \\
=|\operatorname{det} Q|^{1 / 2}|\sin \phi|^{-n / 2} \int_{R^{n}} \prod_{j=1}^{p+q}\left|\frac{\sin \phi}{m_{j}}\right|^{1 / 2} \varepsilon(k(\phi))^{ \pm 1} e^{ \pm i \phi / 2} \\
\quad \times \exp \left[-\pi m_{j}\left(y_{j}^{2}+2 i y_{j} e^{ \pm i \phi} z_{j}-\frac{1}{2} e^{ \pm 2 i \phi} z_{j}^{2}\right)\right] f(y) d y .
\end{aligned}
$$


The \pm convention has been to use the top sign for $1 \leq j \leq p$ and the bottom sign for $p<j \leq p+q$. Since

$$
|\operatorname{det} Q|=\prod_{j=1}^{p+q} m_{j}, \text { we get } I(r(k(\phi), Q) f)(z)=\varepsilon(k(\phi))^{p-q} \sqrt{e^{i \phi(p-q)}} I(f)\left(e^{ \pm i \phi} z\right)
$$

where $e^{ \pm i \phi} z=\left(e^{i \phi} z_{1}, \cdots, e^{i \phi} z_{p}, e^{-i \phi} z_{p+1}, \cdots, e^{-i \phi} z_{p+q}\right)$. Now when $f \in \mathscr{S}\left(\boldsymbol{R}^{n}\right)$, $I(f)$ is holomorphic in $\boldsymbol{C}^{n}$, i.e.,

$$
I(f)(z)=\sum_{\nu \geq 0} c_{\nu} z^{\nu_{1}} \cdots z_{p+q}^{\nu_{p+q}}
$$

Thus

$$
I(f)\left(e^{ \pm i \phi} z\right)=\sum c_{\nu} e^{i \phi\left(\nu_{1}+\cdots+\nu_{p}-\nu_{p+1}-\cdots-\nu_{p+q)}\right.} z_{1}^{\nu_{1}} \cdots z_{p+q}^{\nu_{p+q}} .
$$

To ask that $f$ have the first spherical property is thus to ask that $I(f)$ consist only of terms such that $(p-q)+2\left(\nu_{1}+\cdots+\nu_{p}-\nu_{p+1}-\cdots-\nu_{p+q}\right)$ $=\kappa$. To complete the proof requires a simple computation showing that $I\left(f_{\nu}\right)(z)=c_{\nu} z_{1}^{\nu_{1}} \cdots z_{p+q}^{\nu_{p+q}} \quad\left(\right.$ where $c_{\nu}=\prod_{j=1}^{p+q} \sqrt{2 / m_{j}}\left(-i \sqrt{\pi m_{j}}\right)^{\nu_{j}}$ is of no particular importance to us).

Remarks. 1) When $Q$ is not diagonal, one need only know the matrix which diagonalizes it. (Indeed, we could have assumed $m_{j}=1$ in the proof above, but it would not have simplified the proof.) 2) The first spherical property is not restricted to Schwartz functions. The transform $f \rightarrow I(f)$ can be seen to be a unitary map from $L^{2}\left(\boldsymbol{R}^{n}\right)$ to a Hilbert space of entire functions on $C^{n}$. Thus any series of the form (1.16) which is convergent in $L^{2}\left(\boldsymbol{R}^{n}\right)$, will have the first spherical property. (Reference: Igusa, [6, p. 31-36].)

(6) We have the ingredients now to do a 'simple' example: the theta functions for a $1 \times 1$ matrix.

Let $n=1$ and ${ }^{t} x Q y=2 x y$, so that

$$
\left(r(\sigma, Q) f(x)=\left\{\begin{array}{l}
|a|^{1 / 2} e\left(a b x^{2}\right) f(a x) \quad c=0 \\
\sqrt{\frac{2}{c}} \int_{-\infty}^{\infty} e\left[\frac{a x^{2}-2 x y+d y^{2}}{c}\right] f(y) d y \quad c \neq 0
\end{array} .\right.\right.
$$

In particular,

$$
\left(r\left(\sigma_{z}, Q\right) f\right)(x)=v^{1 / 4} e\left(u x^{2}\right) f\left(v^{1 / 2} x\right) .
$$

According to Theorem 1.9, the only function with the first spherical property for weight $\kappa / 2=\nu+1 / 2$, is the Hermite function 


$$
f_{\nu}(x)=H_{\nu}(2 \sqrt{2 \pi} x) \exp \left(-2 \pi x^{2}\right)
$$

so

$$
\left(r\left(\sigma_{z}, Q\right) f_{\nu}\right)(x)=v^{1 / 4} H_{\nu}(2 \sqrt{2 \pi v} x) e\left(z x^{2}\right) .
$$

For $0<r \in Z$, let $L=r Z$, so that $L^{*}=Z / 2 r$ and $v(L)=r$. Then for $h$, $k \in\left\{1 / 2 r, 2 / 2 r, \cdots, 2 r^{2} / 2 r\right\} \simeq L^{*} / L$, Proposition 1.1 gives

$$
c(h, k)_{r}=\left\{\begin{array}{l}
\delta_{h, a k} e(a b h k) \quad c=0 \\
\frac{1}{\sqrt{2 c} r} \sum_{s=1}^{|c|} e\left[\frac{1}{c}\left(a(h+r s)^{2}-2 k(h+r s)+d k^{2}\right)\right] \quad c \neq 0
\end{array}\right.
$$

Moreover, $c L^{*} \subset L$ if and only if $2 r^{2} \mid c$, and $c\langle x, x\rangle$ is even for all $x \in L^{*}$ if and only if $4 r^{2} \mid c$. Thus $D=2 r^{2}$ and $\Gamma_{0}(4 N)=\Gamma_{0}\left(4 r^{2}\right)$. As a function $\omega: L^{*} / L \rightarrow C$ with the first permutation property, we choose

$$
\omega(k)= \begin{cases}0 & \text { if } k \notin Z \\ \psi(k) & \text { if } k \in Z\end{cases}
$$

where $\psi$ is a character mod $r$. One easily sees that, for any $f \in \mathscr{S}(\boldsymbol{R})$,

$$
\theta(f, \omega)=\sum_{m=-\infty}^{\infty} \psi(m) f(m)
$$

(c.f. Theorem 1.2). In our case we get a function of $z$ :

$$
\theta\left(z, f_{\nu}, \psi\right)=v^{-(2 \nu+1) / 4} \theta\left(r\left(\sigma_{z}, Q\right) f_{\nu}, \omega\right)=v^{-\nu / 2} \sum_{m=-\infty}^{\infty} \psi(m) H_{\nu}(2 \sqrt{2 \pi v} m) e\left(m^{2} z\right)
$$

Notice that $\theta\left(z, f_{\nu}, \psi\right)$ vanishes identically unless $\psi(-1)=(-1)^{\nu}$. As a consequence of Theorem 1.5 and its corollaries, we have the following:

Theorem 1.10. (i) Let $\theta(z, h, r)=\sum_{m \equiv h(r)} e\left(m^{2} z\right)$. Then

$$
\begin{aligned}
& \text { (1) } \theta\left(-1 / 4 r^{2} z, h, r\right)=(-2 i z)^{1 / 2} \sum_{k=1}^{r} e(h k / r) \theta(z, k, r) \\
& \text { (2) } \theta(i v, h, r)=\left\{\begin{array}{ll}
1+O\left(v^{-1 / 2}\right) & h=0 \\
O\left(v^{-1 / 2}\right) & h \neq 0
\end{array} \quad 0<v<\infty\right.
\end{aligned}
$$

(ii) Let $\theta_{1, \nu}(z ; 1)=v^{-\nu / 2} \sum_{m=-\infty}^{\infty} H_{\nu}(2 \sqrt{2 \pi v} m) e\left(m^{2} z\right)$. Then

$$
\begin{aligned}
& \text { (1) } j(\gamma, z)^{-(2 \nu+1)} \theta_{1, \nu}(\gamma z ; 1)=\left(\frac{-1}{d}\right)^{\nu} \theta_{1, \nu}(z ; 1) \quad \gamma \in \Gamma_{0}(4) \\
& \text { (2) } \theta_{1, \nu}(-1 / 4 z ; 1)=i^{\nu}(-2 i z)^{\nu+1 / 2} \theta_{1, \nu}(z ; 1)
\end{aligned}
$$


(iii) Let $\psi$ be a character $\bmod r$, with $\psi(-1)=(-1)^{\nu}, \nu=0$ or 1 . Recall that $\psi_{\nu}(d)=\psi(d)(-1 / d)^{\nu}$, an even character. Define

$$
h_{\psi}(z)=\frac{1}{2} \sum_{m=-\infty}^{\infty} \psi(m) m^{\nu} e\left(m^{2} z\right) .
$$

Then

(1) $j(\gamma, z)^{-(2 \nu+1)} h_{\psi}(\gamma z)=\psi_{\nu}(d) h_{\psi}(z) \quad \gamma \in \Gamma_{0}\left(4 r^{2}\right)$

(2) $\left.\quad(c z+d)^{-(2 \nu+1) / 2} h_{\psi}(\gamma z)\right|_{z=i \infty}= \begin{cases}\frac{\sqrt{i^{-\operatorname{sgn}(c)}}}{\sqrt{ } 8 c r} \sum_{h=1}^{r} \psi(h) \sum_{s=1}^{|c|}\left[\frac{a}{c}(h+r s)^{2}\right] & \nu=0 \\ 0 \quad \nu=1 . & \gamma \in S L(2, Z)\end{cases}$

Thus

(3) $h_{\psi} \in\left\{\begin{array}{ll}G_{1 / 2}\left(4 r^{2}, \psi\right) & \nu=0 \\ S_{3 / 2}\left(4 r^{2}, \psi_{1}\right) & \nu=1\end{array}\right.$.

(4) If $\psi$ is primitive $\bmod r$, then

$$
\left.h_{\psi}\right|_{(2 \nu+1) / 2} W\left(4 r^{2}\right)=(-i)^{\nu} r^{-1 / 2} g(\psi) h_{\bar{\psi}}
$$

where $g(\psi)=\sum_{h=1}^{r} \psi(h) e(h / r)$ (the Gaussian sum).

(5) If $T$ and $u$ are integers, with $0<T$, and $\psi$ is primitive, $\bmod r$, then

$$
h_{\psi}(T z / 4 r u z+1)(4 r u z+1)^{-(2 \nu+1) / 2}=\frac{(-1)^{\nu}}{2 r T^{\nu+1}} \sum_{m=-\infty}^{\infty} m^{\nu} \xi(m) e\left(m^{2} z / T\right)
$$

where

$$
\xi(m)=\sum_{k=1}^{r} \sum_{g=1}^{T r} \psi(k) e\left(\left(g m+T g k-u g^{2}\right) / T r\right) .
$$

Proof. (i) follows from Corollary 1.7 and easy estimates, while (ii) follows directly from Corollaries 1.6 and 1.7. The first two assertions of (iii) follow from Corollaries 1.6 and 1.8; the third assertion summarizes the first two. Assertions (4) and (5) are easily derived from Corollary 1.7 and Theorem 1.5, respectively.

Remarks. 1) The first two results, especially (i), are purely technicalwe shall refer to them exactly once later in the paper. 2) Except for the explicit formula (1.25), the contents of (iii) appear in Shimura [11, p. 457], with precisely the same notation. In particular, (5) is again a technical statement which will be used once later in the paper. 
The formula (1.25) provides a characterization of those theta functions of weight $1 / 2$ which are cusp forms.

Corollary 1.11. Let $\psi$ be an even character mod $r$. Then $h_{\psi} \in S_{1 / 2}$ $\left(4 r^{2}, \psi\right)$ if and only if

$$
\sum_{h=1}^{r} \psi(h) e\left(A h^{2} / \delta r\right)=0
$$

for all $A=1, \cdots, r$, where $\delta=1$ when $r$ is odd, and $\delta=2$ when $r$ is even.

Proof. The value of $h_{\psi}$ at any cusp is given by (1.25). Since $h_{\psi}$ transforms under $\Gamma_{0}\left(4 r^{2}\right)$, it suffices to consider a finite set of cusps: those for which $c \mid 4 r^{2}$. Set $4 r^{2}=\alpha c$, and consider the cases $\alpha=4 \beta, \alpha=2 \beta(2 \nmid \beta)$, and $2 \nmid \alpha$.

When $\alpha=4 \beta$, we have

$$
\begin{aligned}
\sum_{s=1}^{|c|} e\left[\frac{a}{c}(h+r s)^{2}\right] & =\sum_{s=1}^{|c|} e\left[\beta a\left(h^{2} / r^{2}+2 s h / r+s^{2}\right)\right] \\
& =e\left(\beta a h^{2} / r^{2}\right) \sum_{s=1}^{|c|} e(2 \beta a h s / r) \\
& = \begin{cases}0 & \text { if } r \nmid 2 \beta a \\
|c| e\left(A h^{2} / 2 r\right) & \text { if } 2 \beta a=A r\end{cases}
\end{aligned}
$$

This, with the observation that $2 \mid A$ if $2 \nmid r$, resolves the first case.

When $\alpha=2 \beta$ and $2 \nmid \beta$, then $2 \mid c$, so $2 \nmid a$, and we get

$$
\begin{aligned}
\sum_{s=1}^{|c|} e\left[\frac{a}{c}(h+r s)^{2}\right] & =\sum_{s=1}^{|c|} e\left[\beta a\left(h^{2} / 2 r^{2}+s h / r+s^{2} / 2\right)\right] \\
& =\sum_{s=1}^{|c|} e\left[\beta a\left(h^{2} / 2 r^{2}+s h / r+s / 2\right)\right] \text { since } s^{2} \equiv s \bmod 2 \\
& =\sum_{s=1}^{|c|} e\left[\beta a\left(h^{2} / 2 r^{2}+(2 h+r) s / 2 r\right)\right] \\
& =\left\{\begin{array}{ll}
0 & \text { if } 2 r \nmid \beta a(2 h+r)-\text { i.e., } \quad \text { if } 2 \nmid r \text { or } r \nmid \beta a \\
|c| e\left(A h^{2} / 2 r\right) & \text { if } 2 \mid r \text { and } \beta a=A r
\end{array} .\right.
\end{aligned}
$$

When $2 \nmid \alpha$, we again have $2 \mid c$, so $2 \nmid \alpha$. Then

$$
\begin{aligned}
& \sum_{s=1}^{|c|} e\left[\frac{a}{c}(h+r s)^{2}\right]=\sum_{s=1}^{|c|} e\left[\alpha a\left(h^{2} / 4 r^{2}+s h / 2 r+s^{2} / 4\right)\right] \\
& \quad=e\left(\alpha a h^{2} / 4 r^{2}\right) \sum_{t=1}^{|c| / 2}\left\{e\left[\alpha \alpha\left(2 t h / 2 r+4 t^{2} / 4\right)\right]+e\left[\alpha a(2 t-1) h / 2 r+(2 t-1)^{2} / 4\right]\right\} \\
& \quad=e_{(}^{\prime}\left(\alpha a h^{2} / 4 r^{2}\right) \sum_{t=1}^{|c| / 2}\{e[\alpha a t h / r]+e[\alpha a(t h / r-h / 2 r)]\}
\end{aligned}
$$




$$
\begin{aligned}
& =e\left(\alpha a h^{2} / 4 r^{2}\right) \sum_{t=1}^{|c| / 2} e[\alpha a t h / r]\{1+e[-h \alpha a / 2 r]\} \\
& = \begin{cases}0 \quad \text { if } r \nmid \alpha a \text { or } 2 \nmid h \\
|c| e\left(A H^{2} / r\right) & \text { if } \alpha a=r A \text { and } h=2 H\end{cases}
\end{aligned}
$$

Remark. A different characterization of those $h_{\psi}$ 's which are cusp forms is given in Serre-Stark [10]: $h_{\psi}$ is a cusp form if and only if $\psi$ is not 'totally even'. It is a straightforward, though lengthy, exercise in Gaussian sums to prove the equivalence of the two characterizations (directly, that is, without reference to theta functions or cusp forms).

(7) Returning to the general case, let $O(Q)$ be the orthogonal group of $Q: O(Q)=\left\{\left.g\right|^{t} g Q g=Q\right\}$. Since $\operatorname{det} Q \neq 0$, det $g= \pm 1$ for $g \in O(Q)$. Let $S O(Q)$ denote the connected component of the identity in $O(Q)$, consisting of those matrices $g$ with $\operatorname{det} g=1$.

We define a unitary representation of $S O(Q)$ on $L^{2}\left(R^{n}\right)$ by letting $(p(g) f)(x)=f\left(g^{-1} x\right)$. By definition of $S O(Q), p(g)$ commutes with the Weil representation:

$$
p(g)(r(\sigma, Q) f)=r(\sigma, Q)(p(g) f) .
$$

We wish to state a sharper version of Corollary 1.8, for which we need the following lemma:

LEmma 1.12. Let $L$ be a lattice, $f \in \mathscr{S}\left(\boldsymbol{R}^{n}\right)$ and $g \in G L(n, R)$. Define $\|g\|=\min \{|\lambda| \mid \lambda$ an eigenvalue for $g\}$, and $\langle g\rangle=\prod_{\lambda} \min \{|\lambda|, 1\}$ (the product taken over all eigenvalues of $g$, with multiplicities). Assume that $\operatorname{det} g=1$, and let $\alpha>0$. Then

$$
\sum_{0 \neq x \in L}|f(\alpha g x)|= \begin{cases}O\left(\langle\alpha g\rangle^{-1}\right)+O\left(\alpha^{-n}\right) & \|\alpha g\|<1 \\ O\left(\alpha^{-(n+m)}\|g\|^{-m}\right) & 1<\|\alpha g\|\end{cases}
$$

for any $m$, where $O$ depends only on $f, L$, and $m$.

Proof. Since $f$ is 'rapidly decreasing', we have estimates

$$
f(x)=\left\{\begin{array}{ll}
O(1) & \|x\|<1 \\
O\left(\|x\|^{-(m+n)}\right) & 1<\|x\|
\end{array} .\right.
$$

Thus we need to estimate the number of points of $\alpha g L$ lying inside the unit ball; for the rest, we can estimate the sum by an integral: each term is bounded by an average over a nearby fundamental parallelotope.

For $\|\alpha g\|<1$, the number of points of $\alpha g L$ inside the unit sphere is 
$O\left(\langle\alpha g\rangle^{-1}\right)$, while the rest is $O\left(\alpha^{-n} \int_{\|x\|>1}\|x\|^{-(m+n)} d x\right)=O\left(\alpha^{-n}\right)$. For $\|\alpha g\|>1$, there are no points of $\alpha g L$ inside the unit ball and

$$
\begin{aligned}
\sum_{0 \neq x \in L}|f(\alpha g x)| & =O\left(\alpha^{-n} \int_{\|x\|>\|\alpha g\|}\|x\|^{-(m+n)} d x\right)=O\left(\alpha^{-n} \int_{r>\|\alpha g\|} r^{-(m+1)} d r\right) \\
& =O\left(\alpha^{-(m+n)}\|g\|^{-m}\right) .
\end{aligned}
$$

CoROLLARY 1.13. Let $f$ have the first spherical property for weight $\kappa / 2$. Then for any $g \in S O(Q)$, and $\gamma=\left(\begin{array}{ll}a & b \\ c & d\end{array}\right) \in S L(2, Z)$,

$$
\begin{aligned}
v^{(x-n) / 4}(c z+d)^{-\kappa / 2} \theta(\gamma z, p(g) f, h)= & \sqrt{i}-(p-q) \text { sgnc } c(h, 0)_{\gamma} f(0) \\
& + \begin{cases}O\left(\left\langle g^{-1}\right\rangle^{-1}\right) & v \text { fixed } \\
& \langle g\rangle \rightarrow 0 \\
O\left(v^{-m}\right) & g \text { fixed }\end{cases}
\end{aligned}
$$

for any $m$, where $O$ depends on $f, L, \gamma$ and either $v$ (in the first case) or $g$ and $m$ (in the second). In particular, if $f(0)=0$, then, for each $g, \theta$ is 'rapidly decreasing' at all cusps of $S L(2, Z)$.

Proof. Since $p(g)$ commutes with the Weil representation, $p(g) f$ also has the first spherical property. Also, $p(g) f(0)=f(0)$. From Theorem 1.5 and definitions, we have

$$
\begin{aligned}
\text { LHS }= & \sqrt{\imath}^{-(p-q) \operatorname{sgn}(c)}\left[c(h, 0)_{r} f(0)+c(h, 0)_{r} v^{-n / 4} \sum_{0 \neq x \in L}\left\{r\left(\sigma_{z}, Q\right) p(g) f\right\}(x)\right. \\
& \left.+\sum_{0 \neq k \in L^{*} / L} c(h, k)_{r} v^{-n / 4} \sum_{x \in L}\left\{r\left(\sigma_{z}, Q\right) p(g) f\right\}(x+k)\right] \\
= & \sqrt{\imath}^{-(p-q) \operatorname{sgn}(c)} c(h, 0)_{r} f(0)+O\left(v^{-n / 4} \sum_{0 \neq x \in L^{*}}\left|\left\{r\left(\sigma_{z}, Q\right) p(g) f\right\}(x)\right|\right) \\
= & \sqrt{\imath}^{-(p-q) \operatorname{sgn}(c)} c(h, 0)_{r} f(0)+O\left(\sum_{0 \neq x \in L^{*}}\left|f\left(v^{1 / 2} g^{-1} x\right)\right|\right)=\text { RHS . }
\end{aligned}
$$

Let $K_{Q}$ be a maximal compact subgroup of $S O(Q)$, and let $\phi$ be a character of $K_{Q}$. Given $g \in \mathscr{S}\left(\boldsymbol{R}^{n}\right)$, let

$$
f(x)=\int_{K_{Q}} \bar{\phi}(k)(p(k) g)(x) d k
$$

(where $d k$ is the Haar measure on $K_{Q}$, normalized so that $\int_{K_{Q}} d k=1$ ). Then $f$ has the property

$$
p(k) f=\phi(k) f, \quad k \in K_{Q} .
$$


We say that a function $f$ has the 'second spherical property' with respect to $\phi$, when $f$ satisfies (1.32). (More precisely, $f$ has the property with respect to the group $K_{Q}$ and the character $\phi$, but we shall be fixing $K_{Q}$; in any event $K_{Q}$ is implicitly defined as the domain of the character $\phi$.)

Let $\Gamma_{Q}$ be a discrete subgroup of $S O(Q)$ which leaves $L$ invariant. Then $\Gamma_{Q}$ permutes the elements of $L^{*} / L$. Let $\Gamma_{Q}^{*}$ be the (normal) subgroup of $\Gamma_{Q}$ which fixes $L^{*} / L$; this is obviously of finite index in $\Gamma_{Q}$, since $L^{*} / L$ is a finite group. Let $\chi$ be a character of $\Gamma_{Q}$ which is trivial on $\Gamma_{Q}^{*}$ (i.e., a character on $\left.\Gamma_{Q} / \Gamma_{Q}^{*}\right)$, and let $\omega_{0}: L^{*} / L \rightarrow C$ be any function. Then

$$
\omega(k)=\frac{1}{\left[\Gamma_{Q}: \Gamma_{Q}^{*}\right]} \sum_{\gamma \in \Gamma_{Q} / \Gamma_{Q}^{*}} \bar{\chi}(\gamma) \omega_{0}(\gamma k)
$$

has the property

$$
\omega(\gamma k)=\chi(\gamma) \omega(k) \quad \gamma \in \Gamma_{Q}, k \in L^{*} .
$$

We say that function $\omega$ satisfying (1.33) has the 'second permutation property' for $\Gamma_{Q}$ with character $\chi$. (This definition holds for any character $\chi$ on $\Gamma_{Q}$, but if $\chi$ is not trivial on $\Gamma_{Q}^{*}$, then $\omega \equiv 0$.)

\section{§ 2. Niwa's theta kernel}

(1) We now specialize to the $3 \times 3$ matrix considered by Niwa and Shintani. Let

$$
Q=\frac{2}{N}\left(\begin{array}{lll} 
& 1 & -2 \\
-2 &
\end{array}\right)
$$

a matrix with signature $(2,1)$. Clearly, $\operatorname{det} Q=-32 / N^{3}$. Let

$$
L=4 N Z \oplus N Z \oplus N Z / 4 \text {. }
$$

Then $v(L)=N^{3}$. Also, $L^{*}=Z \oplus Z / 2 \oplus Z / 16,\langle x, x\rangle \in Z / 2 N$ for $x \in L^{*},{ }^{*} D_{\text {c }}=$ $\operatorname{det}\left(\left\langle\lambda_{i}, \lambda_{j}\right\rangle\right)=-32 N^{3}$ (see Proposition 1.1), and $c L^{*} \subset L$ when $c \equiv 0$ mod $4 N$, so $\Gamma_{0}(4 N)$ satisfies the hypotheses of Proposition 1.1 (ii).

(2) As a quadratic form, $Q$ is a discriminant, and is given by the determinant of a matrix:

$$
Q(x)={ }^{t} x Q x=\frac{2}{N}\left(x_{2}^{2}-4 x_{1} x_{3}\right)=\frac{-8}{N}\left|\begin{array}{cc}
x_{1} & x_{2} / 2 \\
x_{2} / 2 & x_{3}
\end{array}\right|
$$

$S L(2, R)$ imbeds in $S O(Q): g(x)=x^{\prime}$ is given by 


$$
\left(\begin{array}{cc}
x_{1}^{\prime} & x_{2}^{\prime} / 2 \\
x_{2}^{\prime} / 2 & x_{3}^{\prime}
\end{array}\right)=g\left(\begin{array}{cc}
x_{1} & x_{2} / 2 \\
x_{2} / 2 & x_{3}
\end{array}\right)^{t} g
$$

More explicitly,

$$
\left(\begin{array}{ll}
a & b \\
c & d
\end{array}\right) \rightarrow\left(\begin{array}{ccc}
a^{2} & a b & b^{2} \\
2 a c & a d+b c & 2 b d \\
c^{2} & c d & d^{2}
\end{array}\right) .
$$

In fact, this imbedding is an isomorphism of $S O(Q)$ with $S L(2, R) / \pm 1$. A natural choice for $K_{Q}$, a maximal compact subgroup of $S O(Q)$, is the image of $K$ from $S L(2, R)$ :

$$
\begin{aligned}
k(\phi) & =\left(\begin{array}{cc}
\cos \phi & \sin \phi \\
-\sin \phi & \cos \phi
\end{array}\right) \rightarrow\left(\begin{array}{ccc}
\cos ^{2} \phi & \cos \phi \sin \phi & \sin ^{2} \phi \\
-2 \sin \phi \cos \phi & \cos ^{2} \phi-\sin ^{2} \phi & 2 \sin \phi \cos \phi \\
\sin ^{2} \phi & -\sin \phi \cos \phi & \cos ^{2} \phi
\end{array}\right) \\
= & \left(\begin{array}{ccc}
\frac{1+\cos 2 \phi}{2} & \frac{\sin 2 \phi}{2} & \frac{1-\cos 2 \phi}{2} \\
-\sin 2 \phi & \cos 2 \phi & \sin 2 \phi \\
\frac{1-\cos 2 \phi}{2} & \frac{-\sin 2 \phi}{2} & \frac{1+\cos 2 \phi}{2}
\end{array}\right)
\end{aligned}
$$

with $0 \leq \phi<2 \pi$. Notice, since $K_{Q} \approx K / \pm 1$, the characters on $K_{Q}$ are of the form $k(\phi) \rightarrow e^{-2 i m \phi}$ for $m \in Z$, Thus, we shall say that a function has the second spherical property for the weight $2 m$, rather than for the character $e^{-2 i m \phi}$.

(3) We shall identify those functions having both a first and second spherical property.

THEOREm 2.1. Let $m$ and $\lambda$ be integers. Then for every positive integer $\mu$ such that $|m| \leq \lambda+\mu$, there is a unique (up to scalar multiplication) function $L_{m, \lambda, \mu}$ such that

$$
f_{m, \lambda, \mu}=L_{m, \lambda, \mu}\left(x_{1}, x_{2}, x_{3}\right) H_{\mu}\left(\frac{\sqrt{8 \pi}}{N}\left(x_{1}+x_{3}\right)\right) \exp \left(\frac{-2 \pi}{N}\left(2 x_{1}^{2}+x_{2}^{2}+2 x_{3}^{2}\right)\right)
$$

has the first spherical property for weight $\kappa / 2=\lambda+1 / 2$, and the second spherical property for weight $2 m$. The only functions with both a first and second spherical property (for weights $\kappa / 2$ and $2 \mathrm{~m}$ ) are linear combinations of the form

$$
\sum_{\mu \geq 0,|m|-\lambda} \alpha_{\mu} f_{m, \lambda, \mu}(x)
$$


The function $L_{m, \lambda, \mu}$ is defined (up to scalar multiple) by

$$
L_{m, \lambda, \mu}(x)=\frac{1}{2 \pi} \int_{0}^{2 \pi} e^{2 m i \phi} L_{\lambda, \mu}\left(k(\phi)^{-1} x\right) d \phi
$$

where $L_{\lambda, \mu}(x)=H_{\nu_{1}}\left(\sqrt{8 \pi / N}\left(x_{1}-x_{3}\right) H_{\nu_{2}}\left(\sqrt{8 \pi / N} x_{2}\right)\right.$ for any choice of $\nu_{1}$ and $\nu_{2}$ such that $\nu_{1}+\nu_{2}-\mu=\lambda$. In particular, we may take

$$
L_{\lambda, \lambda, 0}=\left(x_{1}-i x_{2}-x_{3}\right)^{\lambda} .
$$

Proof. Observe that

$$
Q={ }^{t} T\left(\begin{array}{lll}
1 & & \\
& 1 & \\
& & -1
\end{array}\right) T \quad \text { with } T=\sqrt{\frac{2}{N}}\left(\begin{array}{rrr}
1 & 0 & -1 \\
0 & 1 & 0 \\
-1 & 0 & -1
\end{array}\right) .
$$

In the diagonalized basis, the functions with the first spherical property are linear combination of functions

$$
H_{\nu_{1}}\left(2 \sqrt{\pi} y_{1}\right) H_{\nu_{2}}\left(2 \sqrt{\pi} y_{2}\right) H_{\mu}\left(2 \sqrt{\pi} y_{3}\right) \exp \left(-\pi\left(y_{1}^{2}+y_{2}^{2}+y_{3}^{2}\right)\right)
$$

with, $\nu_{1}+\nu_{2}-\mu=\lambda$, where $y=T x$. Also in this basis, the image of $K$ is

$$
\left(\begin{array}{rr}
\cos \phi & \sin \phi \\
-\sin \phi & \cos \phi
\end{array}\right) \rightarrow\left(\begin{array}{rcc}
\cos 2 \phi & \sin 2 \phi & 0 \\
-\sin 2 \phi & \cos 2 \phi & 0 \\
0 & 0 & 1
\end{array}\right) \quad 0 \leq \phi<2 \pi .
$$

As shown in (1.31), we can produce a function with the second spherical property for $2 m$, without losing the first spherical property, by integrating

$$
\frac{1}{2 \pi} \int_{0}^{2 \pi} e^{2 m i \phi} H_{\nu_{1}}\left(2 \sqrt{\pi}\left(y_{1} \cos 2 \phi-y_{0} \sin 2 \phi\right)\right) H_{\nu_{2}}\left(2 \sqrt{\pi}\left(y_{1} \sin 2 \phi+y_{2} \cos 2 \phi\right)\right) d \phi .
$$

It is an interesting property of Hermite polynomials, provable from the recurrence relations, that this integral depends only on $m$ and the sum $\nu_{1}+\nu_{2}$, up to a scalar multiple which does depend on the choice of $\nu_{1}$ and $\nu_{2}$ (and may equal 0 ).

It is clear from inspection that the integral must equal 0 if $m$ is a half-integer or if $|m|>\nu_{1}+\nu_{2}$. The main result now follows by changing back to the original basis for $Q$, while the particular case follows from the Hermite identity

$$
(x-i y)^{\lambda}=\sum_{\mu=0}^{\lambda}\left(\begin{array}{l}
\lambda \\
\mu
\end{array}\right)(-i)^{\mu} H_{\lambda-\mu}(x) H_{\mu}(y)
$$

i.e. 
$\left(x_{1}-i x_{2}-x_{3}\right)^{\lambda}=\left(\frac{8 \pi}{N}\right)^{-\lambda / 2} \sum_{\mu=0}^{\lambda}\left(\begin{array}{l}\lambda \\ \mu\end{array}\right)(-i)^{\mu} H_{\mu}\left(\frac{\sqrt{8 \pi}}{N} x_{2}\right) H_{\lambda-\mu}\left(\frac{\sqrt{8 \pi}}{N}\left(x_{1}-x_{3}\right)\right)$.

For the rest of this paper, we shall consider a specific function $f$ :

$$
f(x)=\left(x_{1}-i x_{2}-x_{3}\right)^{2} \exp \left(-\frac{2 \pi}{N} \cdot\left(2 x_{1}^{2}+x_{2}^{2}+2 x_{3}^{2}\right)\right)
$$

which has the first spherical property for weight $\kappa / 2=\lambda+1 / 2$, and the second property for weight $2 \lambda$. If we let $L(x)=x_{1}-i x_{2}-x_{3}$, it is easily seen that

$$
f(x)=L(x)^{\lambda} \exp \left(-\left.\frac{4 \pi}{N} L(x)\right|^{2}+\pi Q(x)\right)
$$

and thus

$$
\begin{aligned}
\left(r\left(\sigma_{z}, Q\right) f\right)(x) & =v^{3 / 4} \exp (\pi i u Q(x)) f(\sqrt{v} x) \\
& =v^{\lambda / 2+3 / 4} L(x)^{\lambda} \exp \left(-\frac{4 \pi v}{N}|L(x)|^{2}\right) \exp (\pi i \bar{z} Q(x))
\end{aligned}
$$

(4) We shall now introduce a function $\omega$ having the first and second permutation properties.

Proposition 2.2. Let $\chi$ be an even character $\bmod 4 N$, and define $\chi_{1}$ $=\chi(-1 /)^{\lambda}$. Let $L^{\prime}=Z \oplus N Z \oplus N Z / 4$. (so that $L \subset L^{\prime} \subset L^{*}$ ) and let $\Gamma_{Q}$ $=\left(\begin{array}{ll}2 & \\ & 1 / 2\end{array}\right) \Gamma_{0}(2 N)\left(\begin{array}{cc}1 / 2 & \\ & 2\end{array}\right)$. Define $\omega: L^{*} / L \rightarrow C$ by

1) $\omega(k)=0 \quad$ if $k \notin L^{\prime}$

2) $\omega(k)=\bar{\chi}_{1}\left(k_{1}\right) \quad$ if $k=\left(k_{1}, k_{2}, k_{3}\right) \in L^{\prime}$.

Then $\omega$ has the first permutation property for $\Gamma_{0}(4 N)$ with character $\bar{\chi}_{1}$, and the second permutation property for $\Gamma_{Q}$ with character $\chi^{2}$.

Proof. The first permutation property is obvious, given the simple calculation that $\langle k, k\rangle \in 2 Z$ for $k \in L^{\prime}$. As for the second permutation property, it is a matter of observing that

$$
\left(\begin{array}{ll}
2 & \\
& 1 / 2
\end{array}\right)\left(\begin{array}{ll}
a & b \\
c & d
\end{array}\right)\left(\begin{array}{cc}
1 / 2 & \\
& 2
\end{array}\right)=\left(\begin{array}{cc}
a & 4 b \\
c / 4 & d
\end{array}\right) \rightarrow\left(\begin{array}{ccc}
a^{2} & 4 a b & 16 b^{2} \\
a c / 2 & a d+b c & 8 b d \\
c^{2} / 16 & c d / 4 & d^{2}
\end{array}\right)
$$

leaves $L$ and $L^{\prime}$ invariant, when $\gamma=\left(\begin{array}{ll}a & b \\ c & d\end{array}\right) \in \Gamma_{0}(2 N)$, and that $\bar{\chi}_{1}\left(a^{2} k_{1}+\right.$ 
$\left.4 a b k_{2}+16 b^{2} k_{3}\right)=\chi^{2}(d) \bar{\chi}_{1}\left(k_{1}\right)$ for $\left(k_{1}, k_{2}, k_{3}\right) \in L^{\prime}$. (Note that, when $\chi$ has modulus $4 N, \chi^{2}$ has modulus $2 N$.)

Remarks. 1) The largest group leaving $L$ invariant is $\left(\begin{array}{ll}2 & \\ & 1 / 2\end{array}\right) S L(2, Z)$ $\left(\begin{array}{ll}1 / 2 & \\ & 2\end{array}\right)$, which may help explain why we use a conjugate to $\Gamma_{0}(2 N)$, rather than some subgroup of $S L(2, Z)$. 2) If $\Gamma_{Q}=\left(\begin{array}{ll}2 & \\ & 1 / 2\end{array}\right) \Gamma_{0}(2 N)\left(\begin{array}{cc}1 / 2 & \\ & 2\end{array}\right)$, then $\Gamma_{Q}^{*}=\left(\begin{array}{ll}2 & \\ & 1 / 2\end{array}\right) \Gamma(2 N)\left(\begin{array}{cc}1 / 2 & \\ & 2\end{array}\right)$, where $\Gamma(2 N)$ is the principal congruence subgroup of level $N$.

(5) Let $w=\xi+i \eta$ be a complex number with $\eta>0$. Recall how the upper half-plane imbeds in $S L(2, R)$ : specifically,

$$
\sigma_{4 w}=\left(\begin{array}{cc}
2 \eta^{1 / 2} & 2 \xi / \eta^{1 / 2} \\
0 & 1 / 2 \eta^{1 / 2}
\end{array}\right) .
$$

(The presence of the ' 4 ' is explained below.) Define the theta kernel of weight $\kappa / 2(\kappa=2 \lambda+1)$ :

$$
\theta(z, w)=(4 \eta)^{-2} v^{-x / 4} \sum_{x \in L^{\prime}} \bar{\chi}_{1}\left(x_{1}\right)\left\{r\left(\sigma_{z}, Q\right) p\left(\sigma_{4 w}\right) f\right\}(x)
$$

where

$$
L^{\prime}=Z \oplus N Z \oplus N Z / 4 \text {, and } f(x)=\left(x_{1}-i x_{2}-x_{3}\right)^{\lambda} \exp \left(-\frac{2 \pi}{N}\left(2 x_{1}^{2}+x_{2}^{2}+2 x_{3}^{2}\right)\right)
$$

Theorem 2.3. Let $\theta$ be the theta kernel of weight $\kappa / 2(\kappa=2 \lambda+1)$.

Then 1) $\theta \in M_{\kappa / 2}(4 N, \bar{\chi}(N /))$ as a function of $z$

2) $\bar{\theta} \in M_{2 \lambda}\left(2 N, \bar{\chi}^{2}\right)$ as a function of $w$

Proof. In the notation of Corollary 1.6, $\theta(z, w)=(4 \eta)^{-\lambda} \theta\left(z, p\left(\sigma_{4 w}\right) f, \omega\right)$, with $\omega$ defined in Proposition 2.2. The first transformation thus follows immediately from the corollary, since $D=-32 N^{3}$ and $q=1$. The transformation in $w$ is based on Corollary 1.4 and definitions. Everything works because $f$ has the first and second spherical properties, and $\omega$ has the first and second permutation properties. (Corollary 1.4 and the definition of $\Gamma_{Q}$ in Proposition 2.2 explain the ' 4 ' in $\sigma_{4 w}$.)

We can be more explicit as to the transformation in $w$ :

Proposition 2.4. Let $\Lambda(x, w)=(1 / \eta)\left(1 / 4 x_{1}-w x_{2}+4 w^{2} x_{3}\right)$, and $D(x)=$ $(N / 2) Q(x)=x_{2}^{2}-4 x_{1} x_{3}$. For $\sigma=\left(\begin{array}{ll}a & b \\ c & d\end{array}\right) \in S L(2, R)$, let 


$$
\hat{\sigma}=\left(\begin{array}{ccc}
a^{2} & 4 a b & 16 b^{2} \\
\frac{1}{2} a c & a d+b c & 8 b d \\
\frac{1}{16} c^{2} & \frac{1}{4} c d & d^{2}
\end{array}\right) .
$$

Then

$$
\theta \overline{\left(\mid{ }_{2 \lambda} \sigma^{-1}\right)}(z, w)=(4 \eta)^{-\lambda} v^{1 / 2} \sum_{x \in L^{\prime}} \bar{\chi}_{1}\left(x_{1}\right) \Lambda(\hat{\sigma} x, w)^{\lambda} \exp \left[-\frac{4 \pi v}{N}|\Lambda(\hat{\sigma} x, w)|^{2}\right] \cdot e(\bar{z} D(x) / N)
$$

Proof. It is easy to check that $\Lambda(x, w)=\left(p\left(\sigma_{4 w}\right) L\right)(x)$, where $L(x)=x_{1}$ $-i x_{2}-x_{3}$, and the identity

$$
2 x_{1}^{2}+x_{2}^{2}+2 x_{3}^{2}=2|L(x)|^{2}-D(x) .
$$

Since $\hat{\sigma}$ is the image of $\sigma_{2}=\left(\begin{array}{ll}2 & 1 / 2\end{array}\right) \sigma\left(\begin{array}{ll}1 / 2 & \\ & 2\end{array}\right)$ in $S O(Q)$ (see Proposition 2.2 ), and $D(\hat{\sigma} x)=D(x)$, the transformation follows from Corollary 1.4 and a formal computation.

The theta kernel $\theta(z, w)$ is essentially the function $\theta\left(z, \sigma_{4 w}\right)$ in Niwa. (The one difference: we include the factor $(4 \eta)^{-2}$ with the theta kernel; Niwa does not.) Actually, $\theta$ is not the 'right' kernel. As in Niwa, it is necessary to invert both variables, $z$ and $w$.

Proposition 2.5. Let $\left.\right|_{\kappa / 2} W(4 N)$ act on the variable $z$, and $\left.\right|_{2 \lambda} W(2 N)$ act on $w$. Define

$$
\theta^{*}=\left.\theta\right|_{\kappa / 2} W(4 N) \overline{\left.\right|_{2 \lambda} W(2 N)} .
$$

Then

1) $\theta^{*} \in M_{k / 2}(4 N, \chi)$ as a function of $z$

2) $\overline{\theta^{*}} \in M_{2 \lambda}\left(2 N, \chi^{2}\right)$ as a function of $w$.

Moreover,

$$
\theta^{*}(z, w)=\left(32 N^{3}\right)^{-1 / 2} i^{\lambda} v^{-\kappa / 4}(4 \eta)^{-\lambda} \sum_{x \subseteq L_{N}^{*}} \check{\chi}_{1}\left(4 x_{1}\right)\left\{r\left(\sigma_{4 N z}, Q\right) p\left(\sigma_{2 N w}\right) f\right\}(x)
$$

where $\check{\chi}_{1}(m)=\sum_{h=1}^{4 N} \bar{\chi}_{1}(h) e(m h / 4 N)$, and $L_{N}^{*}=Z / 4 \oplus Z / 2 \oplus Z / 4$, the dual lattice to $L_{N}=N Z \oplus N Z \oplus N Z$. Alternatively,

$$
\begin{aligned}
\theta^{*}(z, w)= & 2^{-(2 \lambda+1)} N^{-(\lambda / 2+3 / 4)} i^{\lambda} v^{1 / 2}(4 \eta)^{-\lambda} \sum_{x \in \boldsymbol{Z} \oplus \mathbf{Z} \oplus Z} \check{\chi}_{1}\left(x_{1}\right) \Lambda^{\prime}(x, w)^{\lambda} \\
& \times \exp \left[\frac{-\pi v}{4 N^{2}}\left|\Lambda^{\prime}(x, w)\right|^{2}\right] e\left(\bar{z}\left(x_{2}^{2}-x_{1} x_{3}\right)\right)
\end{aligned}
$$


where

$$
\Lambda^{\prime}(x, w)=\frac{1}{\eta}\left(x_{1}-4 N w x_{2}+4 N^{2} w^{2} x_{3}\right) .
$$

Proof. Statements 1) and 2) are standard results for modular forms (see Theorem 2.3). We proceed with the derivation of the formula (2.14)

$$
\begin{gathered}
\left.\theta\right|_{\kappa / 2} W(4 N)=(4 N)^{-\kappa / 4}(-i z)^{-\kappa / 2} \theta(-1 / 4 N z, w) \text { (definition of } W \text { ) } \\
\left.=(4 N)^{-\kappa / 4}(-i z)^{-\kappa / 2}(4 \eta)^{-\lambda} \sum_{L^{*} / L} \omega(h) \theta\left(-1 / 4 N z, p\left(\sigma_{4 w}\right) f, h\right) \text { (definition of } \theta\right) \\
=(4 N)^{-\kappa / 4}(-i z)^{-\kappa / 2}(4 \eta)^{-\lambda} \sqrt{i}^{-1}(4 N z)^{\kappa / 2}\left(32 N^{3}\right)^{-1 / 2} \\
\quad \times \sum_{L^{* / L}} \omega(h) \sum_{L^{* / L}} e(-\langle h, k\rangle) \theta\left(4 N z, p\left(\sigma_{4 w}\right) f, k\right) \text { (Corollary 1.7) } \\
=(2 N)^{-1 / 2}(4 N)^{\kappa / 4-1} i^{\lambda}(4 \eta)^{-\lambda} \sum_{L^{*} / L} \hat{\omega}(k) \theta\left(4 N z, p\left(\sigma_{4 w}\right) f, k\right)
\end{gathered}
$$

where

$$
\hat{\omega}(k)=\sum_{L^{*} / L} \omega(h) e(-\langle h, k\rangle) .
$$

Here $\omega(h)=\bar{\chi}_{1}\left(h_{1}\right)$ for $h=\left(h_{1}, h_{2}, h_{3}\right) \in L^{\prime}=Z \oplus N Z \oplus N Z / 4$, and $\omega(h)=0$ for $h \notin L^{\prime}$. Moreover, $-\langle h, k\rangle \equiv 4 h_{1} k_{3} / N \bmod Z$ for $h \in L^{\prime}, k \in L^{*}$. Thus $\hat{\omega}(k)=\check{\chi}\left(16 k_{3}\right)$ for $k \in L^{*}$. (Recall that $k_{3} \in Z / 16$.) We have so far derived

$$
\left.\theta\right|_{\kappa / 2} W(4 N)=(2 N)^{-1 / 2}(4 N)^{-1} i^{\lambda}(4 \eta)^{-\lambda} v^{-\kappa / 4} \sum_{L^{*}} \check{\chi}_{1}\left(16 k_{3}\right)\left\{r\left(\sigma_{4 N z}, Q\right) p\left(\sigma_{4 w}\right) f\right\}(k) .
$$

The second inversion is a property of the function $f$ :

$$
\begin{aligned}
\left.\left(\eta^{-\lambda} p\left(\sigma_{4 w}\right) f\right)\right|_{2 \lambda} \overline{W(2 N)} & =(2 N)^{\lambda}(-2 N \bar{w})^{-2 \lambda}\left(\frac{\eta}{2 N|w|^{2}}\right)^{-\lambda} p\left(\sigma_{4(-1 / 2 N w)}\right) f \\
& =\left(\frac{w}{\bar{w}}\right)^{2 \lambda} \eta^{-\lambda} p\left(\left(1 / 2^{-2}\right) \sigma_{2 N w} k(\phi)^{-1}\right) f
\end{aligned}
$$

using Corollary 1.4., where $e^{-i \phi}=w / \bar{w}$. Now the second spherical property for $f$ implies a continuation:

$$
=\eta^{-\lambda} p\left(\left(\begin{array}{ll}
1 / 2 & -2
\end{array}\right) \sigma_{2 N w}\right) f
$$

The matrix $\left(\begin{array}{ll}1 / 2 & -2\end{array}\right)$, which imbeds in $S O(Q)$ as $\left(\begin{array}{ll}1 / 4 & -1\end{array}\right)$, acts on the lattice in a simple way, which results in the formula (2.14). Finally, (2.15) follows from (2.14) and definitions.

Remarks. We have followed Niwa in first defining $\theta$, in part because the character $\bar{\chi}_{1}$ is easier to work with than the Gaussian sum $\check{\chi}_{1}$. However, 
$\theta^{*}$ could have been defined directly, in the following way: let

$$
Q_{4}=\frac{1}{2}\left(\begin{array}{lll} 
& 1 & -2 \\
-2 &
\end{array}\right)
$$

and $f_{4}=\left(x_{1}-i x_{2}-x_{3}\right)^{\lambda} \exp \left(-(\pi / 2)\left(2 x_{1}^{2}+x_{2}^{2}+2 x_{3}^{2}\right)\right)$. Since this is just the original $Q$ and $f$ with $N$ set equal to 4 , it is clear that $f$ has the first and second spherical properties for the weights $\kappa / 2$ and $2 \lambda$. Now let

$$
\begin{aligned}
L & =4 N Z \oplus 2 Z \oplus Z \\
L^{\prime} & =Z \oplus 2 Z \oplus Z \\
L^{*} & =Z \oplus Z \oplus Z / 4 N
\end{aligned}
$$

and define $\omega: L^{*} / L \rightarrow C$ by

$$
\omega(k)=\left\{\begin{array}{ll}
0 & k \notin L^{\prime} \\
\check{\chi}\left(k_{1}\right) & k=\left(k_{1}, k_{2}, k_{3}\right) \in L^{\prime}
\end{array} .\right.
$$

It is easily checked that the group satisfying the hypotheses of Proposition 1.1 (ii) is $\Gamma_{0}(4 N)$; $\omega$ obviously has the first permutation property for $\Gamma_{0}(4 N)$ with character $\chi_{1}$, and it is easily checked that $\omega$ has the second permutation property for $\Gamma_{Q}=\left(\begin{array}{ll}\sqrt{2 N} & \\ & 1 / \sqrt{ } 2 N\end{array}\right) \Gamma_{0}(2 N)\left(\begin{array}{ll}1 / \sqrt{2 N} & \\ & \sqrt{2 N}\end{array}\right)$ with character $\bar{\chi}^{2}$. The reader may verify that, up to a constant multiple (depending on $N$ and $\lambda$ ),

$$
\theta^{*}(z, w) \approx v^{-\kappa / 4}(2 N \eta)^{-\lambda} \sum_{x \in L^{\prime}} \check{\bar{\chi}}_{1}\left(x_{1}\right)\left\{r\left(\sigma_{z}, Q_{4}\right) p\left(\sigma_{2 N w}\right) f_{4}\right\}(x) .
$$

Theorem 2.6. Let $\kappa=2 \lambda+1 \geq 3$, and let $\gamma, \gamma^{\prime} \in S L(2, Z)$. Then

$$
(c z+d)^{-\kappa / 2}\left(c^{\prime} \bar{w}+d^{\prime}\right)^{-2 \lambda} \theta\left(\gamma z, \gamma^{\prime} w\right)= \begin{cases}O\left(v^{(1-\kappa) / 4} \eta^{1-\lambda}\right) & v<\eta^{2} \\ O\left(v^{-(\kappa-3) / 4-m} \eta^{2 m-\lambda}\right) & \eta^{2}<v\end{cases}
$$

for any $m$, where $O$ is independent of $z$ and $w$. The same estimates hold for $\theta^{*}$. When $\kappa=1$ and $\chi$ is not the principal character, the estimates (2.16) again hold for $\theta$ and $\theta^{*}$. When $\kappa=1$ and $\chi$ is the principal character (mod $4 N)$, then the same estimates (2.16) hold for all $\gamma \notin\left(\begin{array}{ll}1 & -1\end{array}\right) \Gamma_{0}(4 N)$. (I.e., $\theta$ is 'rapidly decreasing' at all cusps not $\Gamma_{0}(4 N)$-equivalent to $0, \theta^{*}$ is 'rapidly decreasing' at all cusps not $\Gamma_{0}(4 N)$-equivalent to $i \infty$.) For $r \in\left(\begin{array}{ll}1 & -1\end{array}\right) \Gamma_{0}(4 N)$, the estimate is 


$$
(c z+d)^{-1 / 2} \theta\left(\gamma z, \gamma^{\prime} w\right)=v^{1 / 2} \phi(4 N)\left(32 N^{3}\right)^{-1 / 2}+ \begin{cases}O(\eta) & v<\eta^{2} \\ O\left(v^{1 / 2-m} \eta^{2 m}\right) & \eta^{2}<v\end{cases}
$$

Proof. The first part of the theorem follows from Lemma 1.12 and Corollary 1.13 , on noting that

$$
\left\|\sigma_{\tau w}\right\|=\left\|\sigma_{\tau w}^{-1}\right\|=\left\langle\sigma_{z w}\right\rangle=\left\langle\sigma_{\tau w}^{-1}\right\rangle=O\left(\eta^{-1}\right)
$$

with $O$ depending only on $\tau \in S L(2, R)$. When $\kappa=1$, Corollary 1.13 gives

$$
\left.v^{-1 / \rho}(c z+d)^{-1 / 2} \theta(\gamma z, w)\right|_{z=i \infty}=\sqrt{i}^{-\operatorname{sgn}(c)} \sum_{L^{\prime} / L} \bar{\chi}\left(h_{1}\right) c(h, 0)_{\gamma}
$$

where

$$
\begin{aligned}
c(h, 0)_{\gamma} & =\left(32 N^{3}\right)^{-1 / 2}|c|^{-3 / 2} \sum_{r \in L / c L} e\left[\frac{a}{2 c}\langle h+r, h+r\rangle\right] \\
& =\left(32 N^{3}\right)^{-1 / 2}|c|^{-3 / 2} \sum_{r \in L / c L} e\left[\frac{a}{N c}\left(r_{2}^{2}-4\left(h_{1}+r_{1}\right) r_{3}\right)\right] \\
& =\left(32 N^{3}\right)^{-1 / 2}|c|^{-3 / 2} \sum_{s_{2}=1}^{|c|} e\left(\frac{a N s_{2}^{2}}{c}\right) \sum_{s_{1}, s_{3}=1}^{|c|} e\left[\frac{-a}{c}\left(h_{1}+4 N s_{1}\right) s_{3}\right] .
\end{aligned}
$$

Since $\bar{\chi}_{1}\left(h_{1}\right)=0$ when $\left(h_{1}, 4 N\right)>1$, we need only evaluate $c(h, 0)_{\gamma}$ for $\left(h_{1}, 4 N\right)=1$. In this case

$$
\sum_{s_{1}, s_{3}=1}^{|c|} e\left[\frac{-a}{c}\left(h_{1}+4 N s_{1}\right) s_{3}\right]=\left\{\begin{array}{ll}
|c| & \text { if }(c, 4 N)=1 \\
0 & \text { if }(c, 4 N)>1
\end{array} .\right.
$$

But up to $\Gamma_{0}(4 N)$-equivalence, there is only one cusp for which $(c, 4 N)=1$, the cusp at 0 , corresponding to $\gamma=\left(\begin{array}{ll}1 & -1\end{array}\right)$. We have $c(h, 0)_{\gamma}=\left(32 N^{3}\right)^{-1 / 2}$ for $\gamma=\left(\begin{array}{ll}1 & -1\end{array}\right)$, and thus $\left.v^{-1 / 2} z^{-1 / 2} \theta(-1 / z, w)\right|_{z=i \infty}=\sqrt{\bar{i}^{-1}}\left(32 N^{3}\right)^{-1 / 2} \sum_{h=1}^{4 N} \bar{\chi}(h)$. The last sum is either 0 or $\phi(4 N)$, proving the theorem.

(6) The utility of the theta kernel is that it allows us to pass between forms of half-integral weight and forms of integral weight, via the Petersson inner product.

Proposition 2.7. Let $G \in M_{\kappa / 2}(4 N, \chi)$ and assume that

$$
\Phi(w)=\int_{\Gamma_{0}(4 N) \backslash H} v^{\kappa / 2} G(z) \overline{\theta^{*}(z, w)} d_{0} z
$$

is well defined (i.e., assume that it is absolutely convergent). Then $\Phi \in$ $M_{2 \lambda}\left(2 N, \chi^{2}\right)$, where $\kappa=2 \lambda+1$. Conversely, if $\Phi \in M_{2 \lambda}\left(2 N, \chi^{2}\right)$ and 


$$
G(z)=\int_{\Gamma_{0}(2 N) \backslash H} \eta^{2 \lambda} \Phi(w) \theta^{*}(z, w) d_{0} w
$$

is an absolutely convergent integral, then $G \in M_{\kappa / 2}(4 N, \chi)$.

Proof. This is immediate from Proposition 2.5.

Remark. We are not asserting that $\theta^{*}$ gives any kind of isomorphism -i.e.,

and

$$
k\left(z, z^{\prime}\right)=\int_{\Gamma_{0}(4 N) \backslash I I} \eta^{2 \lambda} \theta^{*}(z, w) \theta^{*}\left(z^{\prime}, w\right) d_{0} w
$$

$$
k^{\prime}\left(w, w^{\prime}\right)=\int_{\Gamma_{0}(4 . V) \backslash I I} v^{\kappa / 2} \theta^{*}(z, w) \overline{\theta^{*}\left(\overline{\left.z, w^{\prime}\right)}\right.} d_{0} z
$$

are not, in general, self-reproducing kernels. (The first integral is not even absolutely convergent.)

The question is: When do these integrals converge, and what are the growth properties of the resulting functions? In order to answer this, we introduce some notation. For a discrete subgroup $\Gamma \subset S L(2, Z)$, of finite index, with character $\omega$, and for a weight $k$, let

$$
P^{m}=P_{k}^{m}(\Gamma, \omega)=\left\{G \in M_{k}(\Gamma, \omega) \mid\left(\left.G\right|_{k} \gamma\right)(z)=O\left(v^{m}\right) \quad v \rightarrow \infty,\right.
$$

Let also for all $\gamma \in S L(2, Z)$, uniformly in $u\}$

$$
P^{m-}=\bigcup_{i<m} P^{\lambda}, \quad P=\bigcup_{\lambda} P^{\lambda}, \quad \text { and } \mathscr{S}=\bigcap_{\lambda} P^{\lambda}
$$

(Thus forms in a $P$-space show polynomial growth-or decay-at all cusps, while forms in the space $\mathscr{S}$ are 'rapidly decreasing'-essentially, they are cusp forms.)

Proposition 2.8. Let $\kappa=2 \lambda+1 \geq 3$. Then (2.18) is absolutely convergent for all $G \in P_{\kappa / 2}(4 N, \chi)$, and (2.19) is absolutely convergent for all $\Phi \in P_{2 \lambda}^{-\lambda-}\left(2 N, \chi^{2}\right)$. We have

$$
\begin{aligned}
& \theta^{*}: P_{\kappa / 2}^{m}(4 N, \chi) \rightarrow P_{2 \lambda}^{\max \{2 m, 1-\lambda\}}\left(2 N, \chi^{2}\right) \quad \text { via }(2.18) \\
& \theta^{*}: P_{2 \lambda}^{m}\left(2 N, \chi^{2}\right) \rightarrow P_{\kappa / 2}^{m / 2}(4 N, \chi) \quad \text { for } m<-\lambda \quad \text { via }(2.19)
\end{aligned}
$$

Proof. This follows easily from Theorem 2.6 and the location of the standard fundamental domain for $S L(2, Z)$. Let $G \in P_{\kappa / 2}^{m}(4 N, \chi)$. Then

$$
\begin{aligned}
\left(\left.\Phi\right|_{22} \gamma^{\prime}\right)(w) & =\int_{\Gamma_{0}(4, N) \backslash H} v^{\kappa / 2} G(z) \overline{\left(\left.\theta^{*}\right|_{2 \lambda} \gamma^{\prime}\right)(z, w)} d_{0} z \\
& =\sum_{\gamma \in S L(2, Z) \backslash \Gamma_{0}\left(4 N^{*}\right)} \int_{S L(2, Z) \backslash H} v^{\kappa / 2}\left(\left.G\right|_{\kappa / 2} \gamma\right)(z) \overline{\left(\theta^{*} \overline{\left.\left.\left.\right|_{2 \lambda} \gamma^{\prime}\right|_{\kappa / 2} \gamma\right)(z, w)} d_{0} z\right.}
\end{aligned}
$$




$$
\begin{aligned}
& =O\left(\int_{1}^{\eta^{2}} v^{\kappa / 2} v^{m} v^{(1-\kappa) / 4} \eta^{1-\lambda} d v / v^{2}+\int_{\eta^{2}}^{\infty} v^{\kappa / 2} v^{m} v^{-(\kappa-3) / 4-h} \eta^{2 h-\lambda} d v / v^{2}\right) \\
& =O\left(\eta^{1-\lambda} \int_{1}^{\eta^{2}} v^{(\lambda-1) / 2+m-1} d v+\eta^{2 h-\lambda} \int_{\eta^{2}}^{\infty} v^{\lambda / 2+m-h-1} d v\right) \\
& =O\left(\eta^{1-\lambda}\left(\eta^{2((\lambda-1) / 2+m)}-1\right)-\eta^{2 h-\lambda} \eta^{2(\lambda / 2+m-h)}\right) \\
& =O\left(\eta^{2 m}+\eta^{1-\lambda}+\eta^{2 m}\right)=O\left(\eta^{2 m}+\eta^{1-\lambda}\right) .
\end{aligned}
$$

Likewise, if $\Phi \in P_{2 \lambda}^{m}\left(2 N, \chi^{2}\right)$, then

$$
\begin{aligned}
\left(\left.G\right|_{\kappa / 2} \gamma\right)(z) & =\int_{\Gamma_{0}(2 N) \backslash H} \eta^{2 \lambda} \Phi(w)\left(\left.\theta^{*}\right|_{\kappa / 2} \gamma\right)(z, w) d_{0} w \\
& =\sum_{\gamma^{\prime} \in S L(2, \boldsymbol{Z}) \backslash \Gamma_{0}(2 N)} \int_{S L(2, Z) \backslash H} \eta^{2 \lambda}\left(\left.\Phi\right|_{2 \lambda} \gamma^{\prime}\right)(w)\left(\left.\theta^{*}\right|_{\kappa / 2} \gamma \mid \overline{2_{2} \gamma^{\prime}}\right)(z, w) d_{0} w \\
& =O\left(\int_{1}^{\sqrt{v}} \eta^{2 \lambda} \eta^{m} v^{-\kappa / 4-h+3 / 4} \eta^{2 h-2} d \eta / \eta^{2}+\int_{\sqrt{v}}^{\infty} \eta^{2 \lambda} \eta^{m} v^{(1-\kappa) / 4} \eta^{1-\lambda} d \eta / \eta^{2}\right) \\
& =O\left(v^{-((\lambda-1) / 2+h)} \int_{1}^{\sqrt{v}} \eta^{\lambda+m+2 h-2} d \eta+v^{-\lambda / 2} \int_{\sqrt{v}}^{\infty} \eta^{\lambda+m-1} d \eta\right) \\
& =O\left(v^{-((\lambda-1) / 2+h)}\left(v^{(\lambda+m+2 h-1) / 2}-1\right)+v^{-\lambda / 2} v^{(\lambda+m) / 2}\right)
\end{aligned}
$$

(but we must have $\lambda+m<0$ for the second integral to converge)

$$
=O\left(v^{m / 2}-v^{-((\lambda-1) / 2+h)}+v^{m / 2}\right)=O\left(v^{m / 2}\right)
$$

since $h$ may be taken arbitrarily large.

The case $\kappa=1$ is complicated by the fact that $f(0) \neq 0$. The reader may convince himself of the following:

Proposition 2.9. Let $\kappa=1$. If $\chi$ is not the principal character mod $4 N$, then the conclusions of Proposition 2.8 hold in this case also. If $\chi$ is the principal character, then the conclusions still hold, provided we place one restriction on the functions $G$ :

$$
\int_{1}^{\infty}|G(z)| d v / v<C
$$

for some constant $C$ (depending on $G$, and not on $u$ ).

(7) As a simple example of a lifting via the theta kernel $\theta^{*}$, we consider an Eisenstein series in the case that $\chi_{1}$ is a primitive character $\bmod 4 N$.

Proposition 2.10. Let $\kappa=2 \lambda+1 \geq 3$, and define

$$
E_{\kappa / 2}(z, s)=E_{\kappa / 2}(z, s, 4 N, \chi)=\sum_{\gamma \in \Gamma_{\infty} \backslash \Gamma_{0}(4 N)} \bar{\chi}(d) \frac{\operatorname{Im}(\gamma z)^{s}}{j(\gamma, z)^{x / 2}} \in P_{\kappa / 2}(4 N, \chi)
$$




$$
E_{2 \lambda}(w, 2 s)=E_{2 \lambda}\left(w, 2 s, 2 N, \chi^{2}\right)=\sum_{\gamma \in \Gamma_{\infty} \backslash \Gamma_{0}(2 N)} \bar{\chi}^{2}(d) \frac{\operatorname{Im}(\gamma z)^{2 s}}{(c w+d)^{2 \lambda}} \in P_{2 \lambda}\left(2 N, \chi^{2}\right)
$$

Assume that $\chi_{1}=\chi(-1 /)^{2}$ is primitive $\bmod 4 N$. Then

$$
\int_{\Gamma_{0}(4 N) \backslash H} v^{\kappa / 2} E_{\kappa / 2}(z, s) \overline{\theta^{*}(z, w)} d_{0} z=C(s) E_{2 \lambda}(w, 2 s)
$$

where

$$
C(s)=2^{-4 \lambda} i^{\lambda} N^{-(\lambda / 2+3 / 4)}\left(\frac{\pi}{4 N^{2}}\right)^{-(\lambda+s)} \Gamma(\lambda+s) g\left(\chi_{1}\right) L\left(\lambda+2 s, \bar{\chi}_{1}\right)
$$

with

$$
g\left(\chi_{1}\right)=\sum_{h=1}^{4 N} \chi_{1}(h) e(h / 4 N) \quad \text { and } \quad L(t, \omega)=\sum_{n=1}^{\infty} \frac{\omega(n)}{n^{t}}
$$

Proof. We shall go as far as we can before assuming $\chi_{1}$ to be primitive. Using the expression (2.15) for $\theta^{*}$ and the usual unfolding trick with Eisenstein series, we have

$$
\begin{gathered}
\int_{\Gamma_{0}(4 N) \backslash H} v^{\kappa / 2} E_{\kappa / 2}(z, s) \overline{\theta^{*}(z, w)} d_{0} z=C_{\lambda}(4 \eta)^{-\lambda} \int_{0}^{\infty} \int_{0}^{1} v^{(\kappa+1) / 2+s} \sum_{z \oplus \bar{Z} \oplus Z} \overline{\bar{\chi}_{1}(x)} \overline{\Lambda^{\prime}(x, \bar{w})^{\lambda}} \\
\times \exp \left[\frac{-\pi v}{4 N^{2}}\left|\Lambda^{\prime}(x, w)\right|^{2}\right] e\left(-\bar{z}\left(x_{2}^{2}-x_{1} x_{3}\right)\right) d u d v / v^{2}
\end{gathered}
$$

with $C_{\lambda}=2^{-(2 \lambda+1)} N^{-(\lambda / 2+3 / 4)}(-i)^{\lambda}$. The integral over $u$ picks up only those terms for which $x_{2}^{2}-x_{1} x_{3}=0$. We continue

$$
\begin{aligned}
& =C_{\lambda}(4 \eta)^{-\lambda} \int_{0}^{\infty} v^{\lambda+s} \sum_{x_{2}^{2}=x_{1} x_{3}} \check{\chi}_{1}\left(-x_{1}\right) \overline{\Lambda^{\prime}(x, w)^{\lambda}} \exp \left(\frac{-\pi v}{4 N^{2}}\left|\Lambda^{\prime}(x, w)\right|^{2}\right) d v / v \\
& =C_{\lambda}\left(\frac{\pi}{4 N^{2}}\right)^{-(\lambda+s)}(4 \eta)^{-\lambda} \sum_{x_{2}^{2}=x_{1} x_{3}}^{\prime} \check{\chi}_{1}\left(-x_{1}\right) \frac{\overline{\Lambda^{\prime}(x, w)^{\lambda}}}{\left|\Lambda^{\prime}(x, w)\right|^{2(\lambda+s)}} \Gamma(\lambda+s) \\
& =C_{\lambda}\left(\frac{\pi}{4 N^{2}}\right)^{-(\lambda+s)}(4 \eta)^{-\lambda} \sum_{x_{2}^{2}=x_{1} x_{3}}^{\prime} \frac{\check{\chi}_{1}\left(-x_{1}\right)}{\Lambda^{\prime}(x, w)^{2}\left|\Lambda^{\prime}(x, w)\right|^{2 s}} \Gamma(\lambda+s) .
\end{aligned}
$$

When $x_{2}^{2}=x_{1} x_{3}$, and not all three are zero, we may write $x_{1}=\mu m^{2}, x_{2}=$ $-\mu m n, x_{3}=\mu n^{2}$, with $\mu$ square-free, and $(m, n) \neq(0,0)$. The correspondence: $(m, n, \mu) \rightarrow\left(x_{1}, x_{2}, x_{3}\right)$ is $2-1$, so that, formally,

$$
\sum_{x_{2}^{2}=x_{1} x_{3}}^{\prime}=\frac{1}{2} \sum_{\mu=\square \text { free }} \sum_{m, n}^{\prime} .
$$

\section{Now}




$$
\begin{aligned}
\Lambda^{\prime}\left(x,{ }^{\prime} w\right) & =\frac{1}{\eta}\left(x_{1}-4 N w x_{2}+4 N^{2} w^{2} x_{3}\right) \\
& =\frac{1}{\eta}\left(\mu m^{2}+4 N w \mu m n+4 N^{2} w^{2} \mu n^{2}\right) \\
& =\frac{\mu}{\eta}(m+2 N n w)^{2} .
\end{aligned}
$$

The equality continues as

$$
\begin{aligned}
& =C_{\lambda}^{\prime} \eta^{-\lambda} \frac{1}{2}\left\{\sum_{\mu=\square} \sum_{\text {fiee }} \sum_{m, n}^{\prime} \frac{\check{\chi}_{1}\left(-\mu m^{2}\right)}{\left((\mu / \eta)(m+2 N n w)^{2}\right)^{\lambda}}\left|\frac{\mu}{\eta}(m+2 N n w)^{2}\right|^{-2 s}\right\} \\
& =C_{\lambda}^{\prime} \sum_{0<\mu=\square \text { free }} \frac{1}{\mu^{\lambda+2 s}} \sum_{m, n}^{\prime} \frac{\check{\chi}_{1}\left(-\mu m^{2}\right)}{(m+2 N n w)^{2 \lambda}}\left(\frac{\eta}{|m+2 N n w|^{2}}\right)^{2 s}
\end{aligned}
$$

with $C_{\lambda}^{\prime}=C_{\lambda}\left(\pi / 4 N^{2}\right)^{-(\lambda+s)} 4^{-\lambda} \Gamma(\lambda+s)$. We assume now that $\chi_{1}$ is primitive. Then $\check{\chi}_{1}=g\left(\chi_{1}\right) \bar{\chi}_{1}$, and the double sum separates:

$$
\begin{aligned}
& =C_{\lambda}^{\prime} g\left(\chi_{1}\right)\left(\sum_{0<\mu=\square \text { free }} \frac{\bar{\chi}_{1}(-\mu)}{\mu^{\lambda+2 s}}\right)\left(\sum_{m, n}^{\prime} \frac{\bar{\chi}_{1}^{2}(m)}{(m+2 N n w)^{2 \lambda}}\left(\frac{\eta}{|m+2 N n w|^{2}}\right)^{2 s}\right) \\
& =C_{\lambda}^{\prime} g\left(\chi_{1}\right)\left(\sum_{0<\mu=D_{\text {free }}} \frac{\bar{\chi}_{1}(-\mu)}{\mu^{2+2 s}}\right)\left(\sum_{M \neq 0} \frac{\bar{\chi}_{1}^{2}(M)}{M^{2 \lambda}|M|^{s}}\right) \\
& \quad \times\left(\sum_{\substack{(c, d)=1 \\
c=(2 N) \\
0(20) \\
c=0=d=1}} \frac{\bar{\chi}^{2}(d)}{(c w+d)^{2 \lambda}}\left(\frac{\eta}{|c w+d|^{2}}\right)^{2 s}\right) \\
& =C_{\lambda}^{\prime} g\left(\chi_{1}\right) 2 \sum_{m=1}^{\infty} \frac{\bar{\chi}_{1}(-m)}{m^{2+2 s}} \sum_{r \in \Gamma_{\infty} \backslash \Gamma_{0}(2 N)} \frac{\bar{\chi}^{2}(d) \operatorname{Im}(\gamma w)^{2 s}}{(c w+d)^{2 \lambda}} \\
& =C(s) E_{2 \lambda}\left(w, 2 s, 2 N, \chi^{2}\right), \text { as desired. }
\end{aligned}
$$

(8) In this section we prove two key results: First, that, on the imaginary axis in $w, \theta^{*}$ can be written as a 'Poincaré' series, i.e., as a sum over coset representatives of $\Gamma_{\infty}$ in $\Gamma_{0}(4 N)$. This leads to the second result, which is a direct calculation of the Mellin transform of a lifted form. This calculation shows that, at least on the imaginary axis, the theta kernel reproduces the Shimura lift (up to an explicit, multiplicative constant). In the next section we show that, for $\kappa \geq 3$, the theta-kernel lifting of a holomorphic form is holomorphic, so that the identification with the Shimura lift is complete. For $\kappa=1$, there is no Shimura lift, and the theta kernel lifting is not holomorphic. Instead, it is 'equal parts' holomorphic and anti-holomorphic, i.e., of the form $\Phi(w)+\Phi(-\bar{w})$. 
Theorem 2.11.

$$
\begin{aligned}
\theta^{*}(z, i \eta)= & C \sum_{\nu=0}^{\lambda}\left(\begin{array}{c}
\lambda \\
\nu
\end{array}\right)\left(\frac{2}{\pi}\right)^{\nu / 2} \eta^{1-\nu} \sum_{\gamma \in \Gamma_{\infty} \backslash \Gamma_{0}(4 N)} \frac{\bar{\chi}(d)}{(\operatorname{Im} \gamma z)^{\lambda-\nu / 2} j(\gamma, z)^{x}} \\
& \times \sum_{m, n=-\infty}^{\infty} \bar{\chi}_{1}(m) m^{\lambda-\nu} \cdot H_{\nu}(2 \sqrt{2 \pi \operatorname{Im} \gamma z} n) \exp \left(2 \pi i n^{2} \gamma z-\frac{\pi m^{2} \eta^{2}}{4 \operatorname{Im} \gamma z}\right)
\end{aligned}
$$

with $C(-1)^{\lambda} 2^{-4 \lambda} N^{\lambda / 2+1 / 4}$.

Proof. The spherical function $f$ 'evolved' out of the Hermite polynomials via (2.6). It is straightforward to verify that

$$
\theta(z, i \eta)=(4 \eta)^{-i}\left(\frac{8 \pi}{N}\right)^{-\lambda / 2} \sum_{\nu=0}^{\lambda}\left(\begin{array}{l}
\lambda \\
\nu
\end{array}\right)(-i)^{\nu} \theta_{1, \nu}(z) \theta_{2, \lambda-\nu}(z, 4 \eta)
$$

where

$$
\begin{aligned}
& \theta_{1, \nu}(z)=v^{-\nu / 2} \sum_{x_{2}=-\infty}^{\infty} H_{\nu}\left(2 \sqrt{2 \pi N v} x_{2}\right) \exp \left(2 \pi i N z x_{2}^{2}\right) \\
& \theta_{2, \nu}(z, 4 \eta)= v^{(1-i) / 2} \sum_{x_{1}, x_{3}=-\infty}^{\infty} \bar{\chi}_{1}\left(x_{1}\right) H_{\nu}\left(2 \sqrt{\frac{2 \pi v}{N}}\left(\frac{x_{1}}{4 \eta}-N \eta x_{3}\right)\right) \\
& \times \exp \left[-2 \pi i u x_{1} x_{3}-\frac{\pi v x_{1}^{2}}{4 N \eta^{2}}-4 \pi N v \eta^{2} x_{3}^{2}\right] .
\end{aligned}
$$

(The notation here is identical with that in Niwa.) Using Theorem 1.10, we have an inversion

$$
\theta_{1 . \nu}(-1 / 4 N z)=N^{\nu / 2} \theta_{1 . \nu}(-1 / 4 z ; 1)=i^{\nu}(-2 i z)^{\nu+1 / 2} N^{\nu / 2} \theta_{1 . \nu}(z ; 1)
$$

where

$$
\theta_{1, \nu}(z ; 1)=v^{-\nu / 2} \sum_{x_{2}=-\infty}^{\infty} H_{\nu}\left(2 \sqrt{2} 2 \pi v x_{2}\right) \exp \left(2 \pi i z x_{2}^{2}\right)
$$

is just $\theta_{1, \nu}(z)$ for $N=1$. In particular, quoting Theorem 1.10,

$$
\theta_{1, \nu}(z ; 1)=\left(\frac{-1}{d}\right)^{\nu} j(\gamma, z)^{-(2 \nu+1)} \theta_{1, \nu}(\gamma z ; 1), \quad \gamma \in \Gamma_{0}(4) .
$$

As for $\theta_{2, \nu}$, Poisson summation on $x_{3}$ gives

$$
\begin{aligned}
\theta_{2, \nu}(z, 4 \eta)= & \left(\frac{8 \pi}{N}\right)^{(\nu+1) / 2}(2 \pi)^{-1 / 2} i^{\nu}(4 \eta)^{-\nu-1} v^{-\nu} \sum_{x_{1},<_{3}=-\infty}^{\Sigma} \bar{\gamma}_{1}\left(x_{1}\right)\left(x_{1} \bar{z}+x_{3}\right)^{\nu} \\
& \times \exp \left(\frac{-\pi}{4 N \eta^{2} v}\left|x_{1} z+x_{3}\right|^{2}\right) .
\end{aligned}
$$

It is straightforward to verify the inversion 


$$
\begin{aligned}
\theta_{2, \lambda-\nu} & (-1 / 4 N z, 4 \eta)=\left(\frac{8 \pi}{N}\right)^{(\lambda-\nu+1) / 2}(2 \pi)^{-1 / 2}(-i z)^{\lambda-\nu}(4 \eta)^{-\lambda+\nu-1} v^{-\lambda+\nu} \\
& \times \sum \bar{\chi}_{1}\left(x_{1}\right)\left(x_{1}+4 N x_{3} \bar{z}\right)^{\lambda-\nu} \exp \left(\frac{-\pi}{16 N^{2} \eta^{2} v}\left|x_{1}+4 N x_{3} z\right|^{2}\right)
\end{aligned}
$$

Now

$$
\begin{aligned}
v^{-\lambda+\nu} & \sum_{x_{1} x_{3}=-\infty}^{\infty} \bar{\chi}_{1}\left(x_{1}\right)\left(x_{1}+4 N x_{3} \bar{z}\right)^{\lambda-\nu} \exp \left(\frac{-\pi}{16 N^{2} \eta^{2} v}\left|x_{1}+4 N x_{3} z\right|^{2}\right) \\
= & \sum_{\substack{c, d)=1 \\
c \geq 0 \\
c=0(4 N) \\
c=0=>d=1}} \sum_{m=-\infty}^{\infty} \bar{\chi}_{1}(m d)\left(\frac{m c \bar{z}+m d}{v}\right)^{\lambda-\nu} \exp \left(\frac{-\pi m^{2}}{16 N^{2} \eta^{2} v}|c z+d|^{2}\right) \\
= & \sum_{r \in \Gamma \infty \backslash \Gamma 0(4 N)} \bar{\chi}_{1}(d)\left(\frac{c \bar{z}+d}{v}\right)^{\lambda-\nu} \sum_{m=-\infty}^{\infty} \bar{\chi}_{1}(m) m^{\lambda-\nu} \exp \left(\frac{-\pi m^{2}}{16 N^{2} \eta^{2} \operatorname{Im} \gamma z}\right) .
\end{aligned}
$$

Since

$$
\begin{aligned}
\bar{\chi}_{1}(d)\left(\frac{c \bar{z}+d}{v}\right)^{\lambda-\nu} & =\bar{\chi}_{1}(d)\left(\frac{-1}{d}\right)^{\lambda+\nu} j(\gamma, z)^{2(\nu-\lambda)}(\operatorname{Im} \gamma z)^{\nu-\lambda} \\
& =\bar{\chi}(d)\left(\frac{-1}{d}\right)^{\nu} j(\gamma, z)^{2(\nu-\lambda)}(\operatorname{Im} \gamma z)^{\nu-\lambda}
\end{aligned}
$$

equation (2.29) gives

$$
\bar{\chi}_{1}(d)\left(\frac{c \bar{z}+d}{v}\right)^{\lambda-\nu} \theta_{1, \nu}(z ; 1)=\bar{\chi}(d) j(\gamma, z)^{-\kappa}(\operatorname{Im} \gamma z)^{\nu-\lambda} \theta_{1, \nu}(\gamma z ; 1) .
$$

The upshot of all this is a rather long formula:

$$
\begin{aligned}
\theta_{1, \nu}(- & 1 / 4 N z) \theta_{2, \lambda-\nu}(-1 / 4 N z, 4 \eta)=\left(\frac{8 \pi}{N}\right)^{(\lambda+1) / 2} \pi^{-1 / 2}(-i z)^{x / 2} i^{\nu}(2 \pi)^{-\nu / 2} \\
& \times N^{\nu} \cdot(4 \eta)^{-\lambda+\nu-1} \sum_{r \in \Gamma_{\infty} \backslash \Gamma_{0}(4 N)} \bar{\chi}(d) j(\gamma, z)^{-x}(\operatorname{Im} \gamma z)^{\nu-\lambda} \theta_{1, \nu}(\gamma z ; 1) \\
& \times \sum_{m=-\infty}^{\infty} \bar{\chi}_{1}(m) m^{\lambda-\nu} \exp \left(-\pi m^{2} / 16 N^{2} \eta^{2} \operatorname{Im} \gamma z\right) .
\end{aligned}
$$

Thus

$$
\begin{aligned}
\left.\theta\right|_{\kappa / 2} W(4 N)(z, i \eta) & =(4 N)^{-\kappa / 4}(-i z)^{-\kappa / 2} \theta(-1 / 4 N z, i \eta) \\
& =2^{1-\lambda} N^{-(\lambda / 2+3 / 4)} \sum_{\nu=0}^{2}\left(\begin{array}{l}
\lambda \\
\nu
\end{array}\right)(2 \pi)^{-\nu / 2} N^{\nu}(4 \eta)^{-2 \lambda+\nu-1} \sum_{\gamma} \quad \text { (as above) } .
\end{aligned}
$$

Finally, the statement of the theorem is derived by applying, in straight-

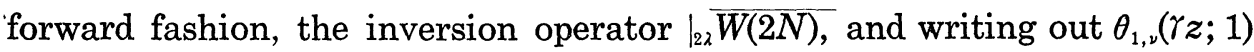
via its definition, (2.28).

We won't use it, but it is interesting to note that the Hermite 
polynomials disappear if one 'inverts' the theta function $\sum \bar{\chi}_{1}(m) m^{\lambda-\nu}$ $\cdot \exp \left(-\pi m^{2} / 16 N^{2} \eta^{2} \operatorname{Im} \gamma z\right)$. The result is as follows:

Corollary.

$$
\begin{aligned}
\left.\theta\right|_{\kappa / 2} & W(4 N)(z, i \eta)=(4 \eta)^{-\lambda} C^{\prime} \sum_{\gamma \in \Gamma_{\infty} \backslash \Gamma_{0}(4 N)} \frac{\bar{\chi}(d) \sqrt{\operatorname{Im} \gamma z}}{j(\gamma, z)^{\kappa}} \\
& \times \sum_{m, n=-\infty}^{\infty} \check{\chi}_{1}(m)(\eta m-2 i n)^{\lambda} \exp \left(2 \pi i n^{2} \gamma z-\pi \operatorname{Im} \gamma z \eta^{2} m^{2}\right)
\end{aligned}
$$

where $C^{\prime}=(-i)^{\lambda} 2^{-\lambda-1} N^{\lambda / 2-3 / 4}$.

THEOREM 2.12. Let $G(z)=\sum_{n=0}^{\infty} a(n) e(n z) \in G_{x / 2}(4 N, \chi)$, with the stipulation that $a(0)=0$ when $\kappa=1$ and $\chi$ is the principal character. Define

$$
\Phi(w)=\int_{\Gamma_{0}(4 N) \backslash H} v^{\kappa / 2} G(z) \overline{\theta^{*}(z, w)} d_{0} z
$$

Then

$$
\Phi(i \infty)=a(0) C_{0}(\lambda) \sum_{m=1}^{\infty} \check{\chi}_{1}(m) m^{-\lambda} \quad(\lambda \geq 1)
$$

with $C_{0}(\lambda)=i^{\lambda} 2^{-2 \lambda} N^{(3 / 2) \lambda-(3 / 4)} \pi^{-\lambda} \Gamma(\lambda)$. When $\lambda=0$,

$$
I(i \infty)=4 N^{1 / 4} a(0) \sum_{m=1}^{\infty} \frac{\chi(m)}{m}
$$

In either case,

$$
\int_{0}^{\infty} \eta^{s-1}(\Phi(i \eta)-\Phi(i \infty)) d \eta=C_{1}(\lambda)(2 \pi)^{-s} \Gamma(s) L\left(s-\lambda+1, \chi_{1}\right) \sum_{n=1}^{\infty} \frac{a\left(n^{2}\right)}{n^{s}}
$$

with

$$
C_{1}(\lambda)=(-1)^{\lambda} 2^{-3 \lambda+2} N^{\lambda / 2+1 / 4} \times \begin{cases}2 & \text { if } \lambda=0 \\ 1 & \text { if } \lambda>0\end{cases}
$$

Thus, for $G \in S_{k / 2}(4 N, \chi), \Phi$ is identical with a constant multiple $\left(C_{1}(\lambda)\right)$ of the Shimura lifting of $G$, on the imaginary axis.

Proof: Using the result of Theorem 2.11, we have

$$
\begin{aligned}
\Phi(i \eta)= & \int_{\Gamma_{0}(4 N) \backslash H} v^{\kappa / 2} G(z) \overline{\theta^{*}(z, i \eta)} d_{0} z \\
= & C \sum_{\nu=0}^{\lambda}\left(\begin{array}{l}
\lambda \\
\nu
\end{array}\right)\left(\frac{2}{\pi}\right)^{\nu / 2} \eta^{1-\nu} \int_{0}^{\infty} \int_{0}^{1} v^{\kappa / 2} G(z) v^{\nu / 2-\lambda} \sum_{m, n=-\infty}^{\infty} \chi_{1}(m) \\
& \times m^{\lambda-\nu} \cdot H_{\nu}(2 \sqrt{2 \pi v} n) \exp \left(-2 \pi i n^{2} \bar{z}-\frac{\pi \eta^{2} m^{2}}{4 v}\right) d_{0} z
\end{aligned}
$$




$$
\begin{aligned}
= & C \sum_{\nu=0}^{2}\left(\begin{array}{c}
\lambda \\
\nu
\end{array}\right)\left(\frac{2}{\pi}\right)^{\nu / 2} \eta^{1-\nu} \int_{0}^{\infty} v^{(\nu-1) / 2} \sum_{n=-\infty}^{\infty} a\left(n^{2}\right) H_{\nu}(2 \sqrt{2 \pi v} n) \\
& \times \exp \left(-4 \pi n^{2} v\right) \cdot \sum_{m=-\infty}^{\infty} \chi_{1}(m) m^{\lambda-\nu} \exp \left(-\pi \eta^{2} m^{2} / 4 v\right) d v / v \\
= & C^{\prime} \sum_{\nu=0}^{\lambda}\left(\begin{array}{c}
\lambda \\
\nu
\end{array}\right)(2 \pi)^{-\nu} \int_{0}^{\infty}\left[a(0) H_{\nu}(0)+\sum_{n \neq 0} a\left(n^{2}\right) H_{\nu}(y n) \exp \left(-n^{2} y^{2} / 2\right)\right] \\
& \times(\eta / y)^{1-\nu} \sum_{m=-\infty}^{\infty} \chi_{1}(m) m^{\lambda-\nu} \exp \left(-2 \pi^{2} m^{2}(\eta / y)^{2}\right) d y / y
\end{aligned}
$$

with $C^{\prime}=2 C(8 \pi)^{1 / 2}=(-1)^{\lambda} 2^{-4 \lambda+2} N^{\lambda / 2+1 / 4}(2 \pi)^{1 / 2}$. It is easy to see that, as $\eta \rightarrow$ $\infty$, the only non-negligible term is that one involving $a(0)$ :

$$
\begin{aligned}
\Phi(i \infty)= & a(0) C^{\prime} \sum_{\nu=0}^{\lambda}\left(\begin{array}{l}
\lambda \\
\nu
\end{array}\right)(2 \pi)^{-\nu} H_{\nu}(0) \int_{0}^{\infty} y^{1-\nu} \sum_{m=-\infty}^{\infty} \chi_{1}(m) m^{\lambda-\nu} \\
& \times \exp \left(-2 \pi^{2} m^{2} y^{2}\right) d y / y .
\end{aligned}
$$

For $\lambda>0$, we 'invert' the theta function (Poisson summation):

$$
\begin{aligned}
y^{1-\nu} & \sum_{-\infty}^{\infty} \chi_{1}(m) m^{\lambda-\nu} \exp \left(-2 \pi^{2} m^{2} y^{2}\right) \\
& =(2 \pi i)^{\nu-\lambda}(2 \pi)^{-1 / 2} y^{-\lambda} \frac{1}{4 N} \sum_{-\infty}^{\infty} \check{\chi}_{1}(m) H_{\lambda-\nu}\left(\frac{m}{4 N y}\right) \exp \left(-m^{2} / 32 N^{2} y^{2}\right)
\end{aligned}
$$

Thus

$$
\begin{aligned}
\Phi(i \infty)= & a(0) C^{\prime \prime} \sum_{\nu=0}^{\lambda}\left(\begin{array}{l}
\lambda \\
\nu
\end{array}\right) i^{\nu} H_{\nu}(0) \int_{0}^{\infty} y^{-\lambda} \sum_{-\infty}^{\infty} \check{\chi}_{1}(m) H_{\lambda-\nu}\left(\frac{m}{4 N y}\right) \\
& \times \exp \left(-m^{2} / 32 N^{2} y^{2}\right) d y / y
\end{aligned}
$$

with $C^{\prime \prime}=C^{\prime}(2 \pi i)^{-\lambda}(2 \pi)^{-1 / 2}(4 N)^{-1}=i^{\lambda} 2^{-5 \lambda} N^{\lambda / 2-3 / 4} \pi^{-\lambda}$. We can now actually sum over $\nu$ :

$$
\left.\sum_{\nu=0}^{\lambda}\left(\begin{array}{l}
\lambda \\
\nu
\end{array}\right) i^{\nu} H_{\nu}(0) H_{\lambda-\nu}\left(\frac{m}{4 N y}\right)=\left(\frac{m}{4 N y}\right)^{\lambda} \quad \text { (see }(2.5)\right)
$$

so

$$
\begin{aligned}
\Phi(i \infty) & =a(0) C^{\prime \prime} \int_{0}^{\infty} \sum_{-\infty}^{\infty} \check{\chi}_{1}(m)\left(\frac{m}{4 N y^{2}}\right)^{\lambda} \exp \left(-m^{2} / 32 N^{2} y^{2}\right) d y / y \\
& =a(0) C^{*} \int_{0}^{\infty} \sum_{m=1}^{\infty} \frac{\check{\chi}_{1}(m)}{m^{\lambda}} y^{\lambda} e^{-y} d y / y
\end{aligned}
$$

with $C^{*}=C^{\prime \prime}(8 N)^{\lambda}=i^{\lambda} 2^{-3 \lambda} N^{(3 / 2) \lambda-(3 / 4)} \pi^{-\lambda}$. The integral gives $\Gamma(\lambda)$, and $C^{*} \Gamma(\lambda)$ $=C_{0}(\lambda)$, as desired. 
For $\lambda=0$, we can dispense with the inversion, and evaluate directly from (2.41):

$$
\begin{aligned}
\Phi(i \infty) & =a(0) C^{\prime} \int_{0}^{\infty} y \sum_{-\infty}^{\infty} \chi(m) \exp \left(-2 \pi^{2} m^{2} y^{2}\right) d y / y \\
& =a(0) C^{\prime} 2^{-1 / 2} \pi^{-1} \Gamma(1 / 2) \sum_{m=1}^{\infty} \frac{\chi(m)}{m} \\
& =4 N^{1 / 4} a(0) \sum_{m=1}^{\infty} \frac{\chi(m)}{m}
\end{aligned}
$$

since $\Gamma(1 / 2)=\pi^{1 / 2}$. Note that the sum must be taken in the principal value' sense, $\sum_{m=1}^{\infty}=\lim _{M \rightarrow \infty} \sum_{m=1}^{M}$, and diverges when $\chi$ is the principal character. That stands to reason: we have already explained that we must assume $a(0)=0$ when $\chi$ is principal. We now have

$$
\begin{aligned}
\Phi(i \eta)-\Phi(i \infty)= & C^{\prime} \sum_{\nu=0}^{\lambda}\left(\begin{array}{l}
\lambda \\
\nu
\end{array}\right)(2 \pi)^{-\nu} \int_{0}^{\infty} \sum_{n \neq 0} a\left(n^{2}\right) H_{\nu}(y n) \exp \left(-n^{2} y^{2} / 2\right)(\eta / y)^{1-\nu} \\
& \times \sum_{-\infty}^{\infty} \chi_{1}(m) m^{2-\nu} \exp \left(-2 \pi^{2} m^{2}(\eta / y)^{2}\right) d y / y .
\end{aligned}
$$

Now

$$
\begin{aligned}
& \int_{0}^{\infty} \eta^{s-1}(\eta / y)^{1-\nu} \sum_{-\infty}^{\infty} \chi_{1}(m) m^{\lambda-\nu} \exp \left(-2 \pi^{2} m^{2}(\eta / y)^{2}\right) d \eta \\
& =y^{s}\left(2 \pi^{2}\right)^{(\nu-s-1) / 2} \sum_{1}^{\infty} \chi_{1}(m) m^{-(s-\lambda+1)} \int_{0}^{\infty} \mu^{(s+1-\nu) / 2} e^{-\mu} d \mu / \mu \\
& =y^{s}\left(2 \pi^{2}\right)^{(\nu-s-1) / 2} L\left(s-\lambda+1, \chi_{1}\right) \Gamma\left(\frac{s+1-\nu}{2}\right) .
\end{aligned}
$$

Thus

$$
\begin{gathered}
\int_{0}^{\infty} \eta^{s-1}(\Phi(i \eta)-\Phi(i \infty)) d \eta=C^{\prime} L\left(s-\lambda+1, \chi_{1}\right) \sum_{\nu=0}^{\lambda}\left(\begin{array}{l}
\lambda \\
\nu
\end{array}\right)(2 \pi)^{-\nu}\left(2 \pi^{2}\right)^{(\nu-s-1) / 2} \\
\quad \times \Gamma\left(\frac{s+1-\nu}{2}\right) \int_{0}^{\infty} y^{s} \sum_{n \neq 0} a\left(n^{2}\right) H_{\nu}(y n) \exp \left(-n^{2} y^{2} / 2\right) d y / y
\end{gathered}
$$

Now

$$
\begin{aligned}
\int_{0}^{\infty} y^{s} \sum_{n \neq 0} a\left(n^{2}\right) H_{\nu}(y n) \exp \left(-n^{2} y^{2} / 2\right) d y / y \\
=\left(\sum_{n=1}^{\infty} \frac{a\left(n^{2}\right)}{n^{s}}\right) \int_{0}^{\infty} y^{s}\left(H_{\nu}(y)+H_{\nu}(-y)\right) e^{-y^{2} / 2} d y / y \\
= \begin{cases}0 \text { if } \nu \text { is odd } \\
2\left(\sum_{1}^{\infty} \frac{a\left(n^{2}\right)}{n^{s}}\right) \int_{0}^{\infty} y^{s-1}(-1)^{\nu}\left(\frac{d^{\nu}}{d y^{\nu}} e^{-y^{2 / 2}}\right) d y \quad \text { if } \nu \text { is even }\end{cases}
\end{aligned}
$$




$$
\begin{aligned}
& =\left\{\begin{array}{l}
0 \\
2\left(\sum_{1}^{\infty} \frac{a\left(n^{2}\right)}{n^{s}}\right)(s-1) \cdots(s-\nu) \int_{0}^{\infty} y^{s-\nu-1} e^{-y^{2} / 2} d y
\end{array}\right. \\
& =\left\{\begin{array}{l}
0 \\
\left(\sum_{1}^{\infty} \frac{a\left(n^{2}\right)}{n^{s}}\right)(s-1) \cdots(s-\nu) \Gamma\left(\frac{s-\nu}{2}\right) 2^{(2-\nu) / 2} .
\end{array}\right.
\end{aligned}
$$

Thus

$$
\begin{gathered}
\int_{0}^{\infty} \eta^{s-1}(\Phi(i \eta)-\Phi(i \infty)) d \eta=C^{\prime} L\left(s-\lambda+1, \chi_{1}\right)\left(\sum_{1}^{\infty} \frac{a\left(n^{2}\right)}{n^{s}}\right) \sum_{\nu \text { even }}\left(\begin{array}{l}
\lambda \\
\nu
\end{array}\right) \\
\times \frac{\Gamma\left(\frac{s+1-\nu}{2}\right) \Gamma\left(\frac{s-\nu}{2}\right)}{2^{\nu+1 / 2} \pi^{s+1}}(s-1) \cdots(s-\nu) .
\end{gathered}
$$

Using the identity $\Gamma(t / 2) \Gamma((t+1) / 2)=2^{1-t} \pi^{1 / 2} \Gamma(t)$, we get

$$
\begin{gathered}
\int_{0}^{\infty} \eta^{s-1}(\Phi(i \eta)-\Phi(i \infty)) d \eta=C^{\prime \prime} L\left(s-\lambda+1, \chi_{1}\right)\left(\sum_{1}^{\infty} \frac{a\left(n^{2}\right)}{n^{s}}\right) \sum_{\nu \text { even }}\left(\begin{array}{l}
\lambda \\
\nu
\end{array}\right) \\
\quad \times \frac{\Gamma(s-\nu)(s-1) \cdots(s-\nu)}{(2 \pi)^{s}}
\end{gathered}
$$

with $C^{\prime \prime}=2 C^{\prime}(2 \pi)^{-1 / 2}=(-1)^{\lambda} 2^{-4 \lambda+3} N^{\lambda / 2+1 / 4}$. From $\Gamma(s-\nu)(s-1) \cdots(s-\nu)$ $=\Gamma(s)$ and $\sum_{\nu \text { even }}\left(\begin{array}{l}\lambda \\ \nu\end{array}\right)=2^{\lambda-1} \times\left\{\begin{array}{ll}1 & \lambda>0 \\ 2 & \lambda=0\end{array}\right.$, we get the result.

Remark. The constant $C_{1}(\lambda)$, which equates Niwa's lifting with Shimura's (at least on the imaginary axis), is different from the constant given in Niwa and quoted in Kojima. The error in Niwa results from the omission of $i^{\nu}$ in his version of (2.27).

(9) In this section we consider the holomorphy of the Niwa lifting. Our proof of holomorphy (for $k \geq 3$ ) is similar to Niwa's in being based on a partial differential equation for $\Phi$, but different in that Theorem 2.12 provides us with stronger, exponential, growth estimates, which eliminate the non-holomorphic solution to the PDE.

Shintani showed that, if $f \in \mathscr{S}\left(\boldsymbol{R}^{n}\right)$, then $F(\sigma, g)=r(\sigma, Q) p(g) f$ satisfied a partial differential equation.

$$
C_{1} F=\left(C_{2}+n\left(\frac{n}{4}-1\right)\right) F
$$

where $C_{1}$ and $C_{2}$ are Casimir operators for $\sigma$ and $g$, respectively. A nor- 
malization is specified indirectly, and must be worked out. In the present case, if we parametrize the variables by $\sigma=\sigma_{z} k(\theta)$ and $g=\sigma_{w} k(\phi)$, then

$$
\begin{aligned}
& C_{1}=4\left[v^{2}\left(\frac{\partial^{2}}{\partial u^{2}}+\frac{\partial^{2}}{\partial v^{2}}\right)-v \frac{\partial^{2}}{\partial u \partial \theta}\right] \\
& C_{2}=\left[\eta^{2}\left(\frac{\partial^{2}}{\partial \xi^{2}}+\frac{\partial^{2}}{\partial \eta^{2}}\right)-\eta \frac{\partial^{2}}{\partial \xi \partial \phi}\right] .
\end{aligned}
$$

The following is an easy consequence:

Proposition 2.13. Assume that $f \in \mathscr{S}\left(\boldsymbol{R}^{3}\right)$ has the first spherical property for weight $\kappa / 2$, and the second for $2 m$. Let $L$ be a set of points such that $N_{L}(r)$, the number of points in $L$ with norm $\leq r$, is polynomially bounded in $r\left(N_{L}(r)=O\left(r^{M}\right)\right.$, for some $\left.M\right)$, and let $P(x)$ be any function of at most polynomial growth. Define

$$
\Theta(z, w)=\eta^{-m} v^{-\kappa / 4} \sum_{x \in L} P(x)\left\{r\left(\sigma_{z}, Q\right) p\left(\sigma_{w}\right) f\right\}(x) .
$$

Then

$$
\begin{aligned}
& 4\left[v^{2}\left(\frac{\partial^{2}}{\partial u^{2}}+\frac{\partial^{2}}{\partial v^{2}}\right)-i \frac{\kappa}{2} v\left(\frac{\partial}{\partial u}+i \frac{\partial}{\partial v}\right)+\frac{\kappa}{4}\left(\frac{\kappa}{4}-1\right)\right] \Theta(z, w) \\
& =\left[\eta^{2}\left(\frac{\partial^{2}}{\partial \xi^{2}}+\frac{\partial^{3}}{\partial \eta^{2}}\right)+2 m i \eta\left(\frac{\partial}{\partial \xi}-i \frac{\partial}{\partial \eta}\right)+m(m-1)-\frac{3}{4}\right] \Theta(z, w) .
\end{aligned}
$$

Theorem 2.14. Suppose $G \in P_{\kappa / 2}(4 N, \chi)$ is holomorphic on $H$.

Let

$$
\Phi(w)=\int_{\Gamma_{0}(4 N) \backslash H} v^{\kappa / 2} G(z) \overline{\theta^{*}(z, w)} d_{0} z .
$$

Then $\Phi$ satisfies the PDE

$$
\left(\eta \frac{\partial}{\partial w}-\lambda i\right) \frac{\partial}{\partial \bar{w}} \Phi=0
$$

Proof. First we note that

$$
\Phi(w)=\int_{S L(2, Z) \backslash H} v^{\kappa / 2} \sum_{\alpha \in \Gamma_{0}(4 N) \backslash S L(2, Z)} G_{\alpha}(z) \overline{\theta_{\alpha}^{*}(z, w)} d_{0} z
$$

where $f_{\alpha}(z)=(c z+d)^{-\kappa / 2} f(\alpha z)$. Observe that $G_{\alpha}$ is still holomorphic on $H$, and that $\theta_{\alpha}^{*}$ still satisfies the conditions of Proposition 2.13, with $m=\lambda$, $\kappa=2 \lambda+1$. That proposition translates to 


$$
\begin{aligned}
\left(\eta^{2} \frac{\partial^{2}}{\partial w \partial \bar{w}}-\lambda i \eta \frac{\partial}{\partial \bar{w}}\right) \Phi & =\int_{S L(2, Z) \backslash H} v_{\alpha \in \Gamma_{0}(4 N) \backslash S L(2, Z)} G_{\alpha}(z) \\
& \times \overline{\left.\left[\eta^{2} \frac{\partial^{2}}{\partial w \partial \bar{w}}+\lambda i \eta \frac{\partial}{\partial w}\right) \theta_{\alpha}^{*}(z, w)\right]} d_{0} z \\
& =\int i^{\kappa / 2} \sum_{\alpha} G_{\alpha} \overline{\left[\left(4 v^{2} \frac{\partial^{L}}{\partial z \partial \bar{z}}-i \kappa v \frac{\partial}{\partial \bar{z}}\right) \theta_{\alpha}^{*}\right.} d_{0} z \\
& =\int i^{\kappa / 2-1} \sum G_{\alpha}\left[4 v \frac{\partial}{\partial z \partial \bar{z}}+i \kappa \frac{\partial}{\partial z}\right] \overline{\theta_{\alpha}^{*}} \frac{d z \wedge d \bar{z}}{-2 i} .
\end{aligned}
$$

Now fix the usual fundamental domain: $S L(2, Z) \backslash H=D=\{z|| z|\geq 1,| \operatorname{Re} z \mid$ $\leq 1 / 2\}$. We use Stokes' Theorem in the form

$$
\int_{D} f(\Delta g) \frac{d z \wedge d \bar{z}}{-2 i}=\int_{D}(\Delta f) g \frac{d z \wedge d \bar{z}}{-2 i}+\int_{\partial D}\left(f \frac{\partial g}{\partial n}-g \frac{\partial f}{\partial n}\right) d \ell
$$

where $\Delta=4\left(\partial^{2} / \partial z \partial \bar{z}\right), \partial / \partial n$ is differentiation along the inward-directed normal, and $d \ell$ is arc length. Thus

$$
\begin{aligned}
\int_{D} v^{\kappa / 2} G_{\alpha}\left(\overline{\Delta \theta_{\alpha}^{*}}\right) \frac{d z \wedge d \bar{z}}{-2 i}= & \int_{D} \Delta\left(v^{\kappa / 2} G_{\alpha}\right) \overline{\theta_{\alpha}^{*}} \frac{d z \wedge d \bar{z}}{-2 i} \\
& +\int_{\partial D}\left(v^{\kappa / 2} G_{\alpha} \frac{\overline{\partial \theta_{\alpha}^{*}}}{\partial n}-\overline{\theta_{\alpha}^{*}} \frac{\partial\left(v^{\kappa / 2} G_{\alpha}\right)}{\partial n}\right) d l .
\end{aligned}
$$

Since $G$ is holomorphic,

$$
\frac{\partial}{\partial \bar{z}}\left(v^{\kappa / 2} G_{\alpha}\right)=\frac{\partial}{\partial \bar{z}}\left(\left(\frac{z-\bar{z}}{2 i}\right)^{\kappa / 2} G_{\alpha}\right)=\frac{\kappa i}{4} v^{\kappa / 2-1} G_{\alpha},
$$

so

$$
\begin{aligned}
\int_{D} v^{\kappa / 2} G_{\alpha}\left(4 \frac{\partial^{2} \overline{\theta_{\alpha}^{*}}}{\partial z \partial \bar{z}}\right) \frac{d z \wedge d \bar{z}}{-2 i}= & \kappa i \int_{D} \frac{\partial}{\partial z}\left(v^{\kappa / 2-1} G_{\alpha}\right) \overline{\theta_{\alpha}^{*}} \frac{d z \wedge d \bar{z}}{-2 i} \\
& +\int_{\partial D}\left(v^{\kappa / 2} G_{\alpha} \frac{\partial \overline{\theta_{\alpha}^{*}}}{\partial n}-\overline{\theta_{\alpha}^{*}} \frac{\partial\left(v^{\kappa / 2} G_{\alpha}\right)}{\partial n}\right) d l
\end{aligned}
$$

Plugging this into (2.53) we get

$$
\begin{aligned}
\left(\eta^{2} \frac{\partial^{2}}{\partial w \partial \bar{w}}-\lambda i \eta \frac{\partial}{\partial \bar{w}}\right) \Phi=\sum_{\alpha}\left[i \kappa \int_{D}\right. & \frac{\partial}{\partial z}\left(v^{\kappa / 2-1} G_{\alpha} \overline{\theta_{\alpha}^{*}}\right) \frac{d z \wedge d \bar{z}}{-2 i} \\
& \left.+\int_{\partial D}\left(v^{\kappa / 2} G_{\alpha} \frac{\partial \overline{\theta_{\alpha}^{*}}}{\partial n}-\overline{\theta_{\alpha}^{*}} \frac{\partial\left(v^{\kappa / 2} G_{\alpha}\right)}{\partial n}\right) d l\right] \\
=\sum_{\alpha}\left[-\frac{\kappa}{2} \int_{\partial D} v^{\kappa / 2-1} G_{\alpha} \overline{\theta_{\alpha}^{*}} d \bar{z}\right. & \left.+\int_{\partial D}\left(v^{\kappa / 2} G_{\alpha} \frac{\partial \overline{\theta_{\alpha}^{*}}}{\partial n}-\overline{\theta_{\alpha}^{*}} \frac{\partial\left(v^{\kappa / 2} G_{\alpha}\right)}{\partial n}\right) d l\right]
\end{aligned}
$$




$$
\begin{aligned}
=\sum_{\alpha}\left[-\frac{\kappa}{2} \int_{\partial D} v^{\kappa / 2-1} G_{\alpha} \overline{\theta_{\alpha}^{*}}(d \bar{z}\right. & \left.+\frac{\partial v}{\partial n} d \ell\right) \\
& \left.+\int_{\partial D} v^{\kappa / 2}\left(G_{\alpha} \frac{\partial \overline{\theta_{\alpha}^{*}}}{\partial n}-\overline{\theta_{\alpha}^{*}} \frac{\partial G_{\alpha}}{\partial n}\right) d \ell\right] .
\end{aligned}
$$

We break the boundary $\partial D$ into the usual four segments. The integrals along the vertical segments are esaily seen to cancel, since

$$
\sum_{\alpha \in \Gamma_{0}(4 N) \backslash S L(2, Z)} G_{\alpha} \overline{\theta_{\alpha}^{*}}(z+1)=\sum_{\alpha \subseteq \Gamma_{0}(4 N) \backslash S L(2, Z)} G_{\alpha} \overline{\theta_{\alpha}^{*}}(z)
$$

and since $\partial v / \partial n=0$ on vertical lines. For the circular segments we have

$$
G_{\alpha} \frac{\partial \overline{\theta_{\alpha}^{*}}}{\partial n}=-G_{\alpha^{\prime}} \frac{\partial \overline{\theta_{\alpha^{\prime}}^{*}}}{\partial n}
$$

and

$$
\overline{\theta_{\alpha}^{*}} \frac{\partial G_{\alpha}}{\partial n}=-\overline{\theta_{\alpha^{\prime}}^{*}} \frac{\partial G_{\alpha^{\prime}}}{\partial n}
$$

where $\alpha=\alpha^{\prime}\left(\begin{array}{rr}0 & -1 \\ 1 & 0\end{array}\right)$. Thus the second integral in (2.54) vanishes by cancellation. We are left with

$$
-\frac{\kappa}{2} \int_{\partial D_{c}} v^{\kappa / 2-1} \sum_{\alpha} G_{\alpha} \overline{\theta_{\alpha}^{*}}\left(d u+\frac{\partial v}{\partial n} d \ell\right)
$$

over the circular segment $\partial D_{c}$, We parametrize this arc by $u=\cos t$, $v=\sin t$, and note that $\partial v / \partial n=\sin t, d \ell=d t$. But then $d u+(\partial v / \partial n) d \ell$ $=0$.

Remark. To apply Stokes' Theorem, we need $\sum_{\alpha} v^{\kappa / 2} G_{\alpha} \overline{\theta_{\alpha}^{*}}$ to vanish at $v=\infty$. In Shintani and Niwa, this is guaranteed by taking $G$ to be a cusp form. But in fact it is guaranteed by the fact (Theorem 2.6) that $\theta^{*}$ is a 'cusp form' (with the usual proviso when $\kappa=1$ and $\chi$ is principal).

Proposition 2.15. Let $\Phi$ be given as in Theorem 2.14. Then $\Phi$ has a Fourier expansion

$$
\Phi(w)=\sum_{n=-\infty}^{\infty} b_{n}(\eta) e(n w)
$$

with

$$
b_{n}(\eta)=B_{n}+C_{n} \int_{1}^{\eta} t^{-2 \lambda} \exp (4 \pi n t) d t
$$


for some constants $B_{n}$ and $C_{n}$.

Proof. From proposition 2.7, we know $\Phi$ is a modular form, so in particular $\Phi(w+1)=\Phi(w)$. Thus $\Phi$ has a Fourier series of the form given, for some functions $b_{n}(\eta)$. Since $\partial \eta / \partial w=-i / 2$ and $\partial \eta / \partial \bar{w}=i / 2$, we see

$$
\partial \Phi / \partial \bar{w}=\sum_{n=-\infty}^{\infty} \frac{i}{2} b_{n}^{\prime}(\eta) e(n w)
$$

so

$$
\begin{aligned}
\partial^{2} \Phi / \partial w \partial \bar{w} & =\sum \frac{i}{2} \frac{\partial}{\partial w}\left[b_{n}^{\prime}(\eta) e(n w)\right]=\sum \frac{i}{2}\left[\frac{-i}{2} b_{n}^{\prime \prime}(\eta)+2 \pi i n b_{n}^{\prime}(\eta)\right] e(n w) \\
& =\sum\left(\frac{1}{4} b_{n}^{\prime \prime}(\eta)-\pi n b_{n}^{\prime}(\eta)\right) e(n w) .
\end{aligned}
$$

Thus

$$
\left(\eta \frac{\partial^{2}}{\partial w \partial \bar{w}}-\lambda i \frac{\partial}{\partial \bar{w}}\right) \Phi=\sum\left(\frac{\eta}{4} b_{n}^{\prime \prime}-\pi n \eta b_{n}^{\prime}+\frac{\lambda}{2} b_{n}^{\prime}\right) e(n w)=0
$$

by Theorem 2.14. We therefore have

$$
b_{n}^{\prime \prime}(\eta)=(4 \pi n-2 \lambda / \eta) b_{n}^{\prime}(\eta)
$$

which has the general solution given by the proposition.

Our next objective is to show that, in the proposition above, $C_{n}=0$ for all $n$, and $B_{n}=0$ for $n<0$. There is one exception, of course: when $\lambda=0$ (weight $1 / 2$ ), $C_{n}$ can be non-zero for $n<0$.

Theorem 2.16. Let $\Phi$ be given as in Theorem 2.14. Then

$$
\Phi(w)=\left\{\begin{array}{l}
\sum_{n=0}^{\infty} B_{n} e(n w) \quad \lambda>0 \\
\sum_{n=0}^{\infty} B_{n}(e(n w)+e(-n \bar{w})) \quad \lambda=0
\end{array}\right.
$$

for some coefficients $B_{n}$. In particular, for $\lambda>0, \Phi$ is holomorphic.

Proof. By Proposition 2.15, $\Phi(w)=\sum_{-\infty}^{\infty} a_{n}(\eta) e(n \xi)$, with

$$
a_{n}(\eta)=B_{n} \exp (-2 \pi n \eta)+C_{n} \exp (-2 \pi n \eta) \int_{1}^{\eta} t^{-2 \lambda} \exp (4 \pi n t) d t .
$$

By the Parseval relation,

$$
\int_{0}^{1}|\Phi(w)|^{2} d \xi=\sum_{-\infty}^{\infty}\left|a_{n}(\eta)\right|^{2}
$$


By Proposition 2.8, $\Phi$ is of (at most) polynomial growth in $\eta$. Therefore, from (2.57), each $a_{n}(\eta)$ must be at most polynomial in $\eta$.

In the case $\lambda=0$, we have

$$
a_{n}(\eta)=\left\{\begin{array}{l}
B_{0}+C_{0} \eta \quad n=0 \\
B_{n}^{\prime} \exp (-2 \pi n \eta)+C_{n}^{\prime} \exp (2 \pi n \eta) \quad n \neq 0
\end{array} .\right.
$$

Thus, for $a_{n}$ to be polynomial in $\eta$, we must have $B_{n}^{\prime}=0$ for $n<0$ and $C_{n}^{\prime}=0$ for $n>0$. Thus

$$
\Phi(w)=B_{0}+C_{0} \eta+\sum_{n=1}^{\infty}\left(B_{n}^{\prime} e(n w)+C_{-n}^{\prime} e(-n \bar{w})\right) .
$$

However, in view of the definition of $\theta^{*}$ for $\lambda=0$, we have $\Phi(w)=\Phi(-\bar{w})$, while from Theorem 2.12 we see that $C_{0}=0$ (i.e., there is no 'pole' at $i \infty$.) The result follows.

In the case $\lambda>0$, observe that

$$
\exp (-2 \pi n \eta) \int_{1}^{\eta} t^{-2 \lambda} \exp (4 \pi n t) d t=O(\exp (2 \pi|n| \eta))
$$

Thus $C_{n}=0$ for $n>0$, and for $n<0$

$$
B_{n}=-C_{n} \int_{1}^{\infty} t^{-2 \lambda} \exp (4 \pi n t) d t
$$

so

$$
a_{-n}(\eta)=-C_{-n} \exp (2 \pi n \eta) \int_{\eta}^{\infty} t^{-2 \lambda} \exp (-4 \pi n t) d t
$$

Restricting to the imaginary axis, we have

$$
\Phi(i \eta)=\sum_{n=0}^{\infty}\left[B_{n} \exp (-2 \pi n \eta)-C_{-n} \exp (2 \pi n \eta) \int_{\eta}^{\infty} t^{-2 \lambda} \exp (-4 \pi n t) d t\right] .
$$

However, from Theorem 2.12, we know

$$
\Phi(i \eta)=\sum_{n=0}^{\infty} b_{n} \exp (-2 \pi n \eta) .
$$

The theorem now rests on the following lemma:

LEMma. Let $\left\{m_{i}\right\}$ be a sequence of real numbers, increasing without bound. Then, for $\lambda>0$,

$$
\sum_{i=1}^{\infty}\left[\beta_{i} \exp \left(-2 \pi m_{i} \eta\right)-\alpha_{i} \exp \left(2 \pi m_{i} \eta\right) \int_{\eta}^{\infty} t^{-2 \lambda} \exp \left(-4 \pi m_{i} t\right) d t\right]=0
$$


holds if and only if $\beta_{i}=\alpha_{i}=0$ for all $i$.

Proof. Let $I$ be the first integer where $\beta=\beta_{I}$ and $\alpha=\alpha_{I}$ are not both 0 , and let $M=m_{I}$. Multiplying by $\exp (2 \pi M \eta)$ gives

$$
0=\beta-\alpha \exp (4 \pi M \eta) \int_{\eta}^{\infty} t^{-2 \lambda} \exp (-4 \pi M t) d t+O\left(e^{-r \eta}\right)
$$

where $r$ is some positive constant. Now simple estimates show

$$
C_{1} \eta^{-2 \lambda}<\exp (4 \pi M \eta) \int_{\eta}^{\infty} t^{-2 \lambda} \exp (-4 \pi M t) d t<C_{2} \eta^{-2 \lambda} \quad(\eta \rightarrow \infty) .
$$

The inequality on the right gives

$$
0=\beta-\alpha O\left(\eta^{-2 \lambda}\right)+O\left(e^{-r \eta}\right) \quad \eta \rightarrow \infty
$$

which proves $\beta=0$. The inequality on the left then shows $\alpha=0$.

Theorems 2.12 and 2.16 imply that the lifting via the theta kernel is essentially (up to $C_{1}(\lambda)$ ) identical with the Shimura lift. We shall state the relationship exactly, but to do so warrants some notation.

Let $t$ be a square-free, positive integer. For $G(z)=\sum_{n=0}^{\infty} a(n) e(n z) \epsilon$ $G_{\kappa / 2}(4 N, \chi)$, with $\kappa=2 \lambda+1 \geqq 3$, define the 'Shimura lift'

$$
S_{t}(G)(w)=\sum_{n=0}^{\infty} A_{t}(n) e(n w)
$$

by

$$
\sum_{n=1}^{\infty} A_{t}(n) n^{-s}=L\left(s-\lambda+1, \chi_{t}\right) \sum_{n=1}^{\infty} a\left(t n^{2}\right) n^{-s}
$$

where $\chi_{t}=\chi(-1 /)^{\lambda}(t /)$ (and $A_{t}(0)$ is the unique value which makes $S_{t}(G)$ into a modular form of weight $2 \lambda)$.

Define $\theta_{t}^{*}(z, w)$ by replacing in $\theta^{*}(z, w)$ every occurrence of $N$ by $N t$, and $\chi_{1}$ by $\chi_{t}$. Define the 'Niwa lift'

$$
N_{t}(G)(w)=\int_{\Gamma_{0}(4 N t) \backslash H} v^{\kappa / 2} G(t z) \theta_{t}^{*}(z, w) d_{0} z
$$

(It is easily checked that this is well-defined.)

TheOREm 2.17. Let the notation be as above. Then

$$
\begin{aligned}
& \text { 1) } N_{t}(G)(w)=C_{t}(\lambda) S_{t}(G)(t w) \text { with } C_{t}(\lambda)=(-1)^{\lambda} 2^{-3 \lambda+2}(t N)^{\lambda / 2+1 / 4} \\
& \text { 2) } a(0) \Gamma(\lambda) \sum_{m=1}^{\infty} \check{\chi}_{t}(m) m^{-\lambda}=i^{\lambda} 2^{-\lambda+2}(N t)^{-\lambda+1} \pi^{\lambda} A_{t}(0)
\end{aligned}
$$


Proof. For $t=1$ this is just a restatement of Theorem 2.12 in light of the fact that the Niwa lifting is holomorphic. For general $t$, we use the reduction $N_{t}(G)(w)=N_{1}\left(G_{t}\right)(w)$ and $S_{t}(G)(t w)=S_{1}\left(G_{t}\right)(w)$, where $G_{t}(z)$ $=G(t z)$. The correct constants come as before. (Keep in mind that the Niwa lifting depends on the level and character assigned to the form being lifted-which for $G_{t}$ is $4 N t$ and $\chi_{t}$.)

Remarks. 1) Niwa showed that $S_{t}(G) \in G_{2 \lambda}\left(2 N, \chi^{2}\right)$, the key question being the level $2 N$. The proof is as follows: we know $N_{t}(G)$ transforms under the group $\Gamma_{0}(2 N t)$ (Proposition 2.7), so $S_{t}(G)(w)=N_{t}(G)(w / t)$ transforms under the group $\Gamma_{0}(2 N, t)=\left\{\left(\begin{array}{ll}a & b \\ c & d\end{array}\right) \mid c \equiv 0(2 N), b \equiv 0(t)\right\}$. But, by construction, $S_{t}(G)$ transforms under $\Gamma_{\infty}$, and between them $\Gamma_{0}(2 N, t)$ and $\Gamma_{\infty}$ generate $\Gamma_{0}(2 N)$.

2) Interestingly, a 'direct' proof doesn't work. That is, one might expect $\theta_{t}^{*}(z, w / t)$ to transform at level $2 N$ in $w$. It doesn't:

$$
\begin{aligned}
\theta_{t}^{*}(z, w / t)= & \text { const. } v^{1 / 2} \eta^{-\lambda} \sum_{z \oplus \bar{Z} \oplus Z} \check{\chi}_{t}(x) \Lambda^{\prime}(x, w)^{\lambda} \\
& \times \exp \left[\frac{-\pi v}{4 N^{2}}\left|\Lambda^{\prime}(x, w)\right|^{2}\right] e\left(\bar{z}\left(x_{2}^{2}-x_{1} x_{3}\right)\right)
\end{aligned}
$$

with $\Lambda^{\prime}(x, w)=1 / \eta\left(x_{1}-4 N w x_{2}+4 N^{2} w^{2} x_{3}\right)$, is not, for general $t$, invariant even for $w \rightarrow w+1$, unless $t \mid 4 N$. It is only when this kernel is integrated against old forms-G(tz)-in $\Gamma_{0}(4 N t)$ that the transformation for $w \rightarrow w+1$ appears.

3) Equation (2.63) can be considerably simplified, using functional equations for the L-series. One derives

$$
A_{t}(0)=\frac{a(0)}{2} L\left(1-\lambda, \chi_{t}\right)
$$

which was conjectured by Cohen [13, p. 194].

\section{$\S 3$. The lifts of theta functions}

In this section, we use Theorem 2.12 to calculate explicitly the lifts of the theta functions of weights $1 / 2$ and $3 / 2$. We find that the theta functions of weight $3 / 2$ lift to Eisenstein series of weight 2 , while theta functions of weight $1 / 2$ lift to integrals of Eisenstein series of weight 2 . In some cases these liftings may be identified also with functions of the form $\log |\eta|$, where $\eta$ is the Dedekind eta function. We then use our knowl- 
edge of Eisenstein series to compute behavior at various cusps. In the last section we shall return to these examples to derive explicit formulae for the inner products of theta functions.

We recall our notation: $\psi$ is a primitive character $\bmod r$ and $\lambda=0$ or 1 , satisfying $\psi(-1)=(-1)^{\lambda}$. We have

$$
h_{\psi}(z)=\frac{1}{2} \sum_{m=-\infty}^{\infty} \psi(m) m^{\lambda} e\left(m^{2} z\right) .
$$

Proposition 3.1 Let $G(z)=h_{\psi}\left(t u^{2} z\right)=\sum_{n=0}^{\infty} a(n) e(n z)$, where $t$ is $a$ square-free positive integer. Then

1) $G \in G_{\lambda+1 / 2}(4 N, \chi)$ if $4 t u^{2} r^{2} \mid 4 N$ and $\chi(m)=\psi(m)\left(\frac{-1}{m}\right)^{2}\left(\frac{t}{m}\right)$

2) $a(n)=\left\{\begin{array}{l}0 \text { if } n \neq t u^{2} m^{2} \\ \psi(m) m^{2} \text { if } n=t u^{2} m^{2} \quad \text { for some } m .\end{array}\right.$

$$
\text { for }(m, 4 N)=1 \text {. }
$$

3) $\chi_{t}(m)=\psi(m)$ for $(m, 4 N)=1$.

4) $\sum_{n=1}^{\infty} A_{t}(n) n^{-s}=L\left(s-\lambda+1, \chi_{t}\right) \sum_{n=1}^{\infty} a\left(t n^{2}\right) n^{-s}$

$$
=\sum_{d \mid 4 N} \mu(d) \psi(d) d^{2-1} \sum_{m=1}^{\infty} \psi(m) m^{2-1} \sigma(m)(u d m)^{-s}
$$

5) $S_{t}(G)(w)=\left\{\begin{array}{c}\sum_{d \mid 4 N} \mu(d) \psi(d) \sum_{m=1}^{\infty} \psi(m) \sigma(m) e(u d m w) \quad \lambda=1 \\ \sum_{d \mid 4 N} \mu(d) \psi(d) \pi i u\left(\int_{i \infty}^{w}+\int_{i \infty}^{\bar{w}}\right) \\ \times\left(\sum_{m=1}^{\infty} \psi(m) \sigma(m) e(u d m \omega) d \omega\right) \quad \lambda=0 .\end{array}\right.$

6) $S_{\tau}(G)=0$ if $\tau \neq t$.

Proof. 1) follows from Theorem 1.10 and standard facts for modular forms. 2) follows from definitions, as does 3). We derive 4) by noting that

$$
L\left(s-\lambda+1, \chi_{t}\right)=\sum_{\substack{(m, 4 N)=1 \\ m \geq 1}} \psi(m) m^{-(s-\lambda+1)}=\sum_{d \mid 4 N} \mu(d) \psi(d) \sum_{m=1}^{\infty} \psi(m)(d m)^{-(s-\lambda+1)} .
$$

Thus

$$
\begin{aligned}
\sum_{n=1}^{\infty} A_{t}(n) n^{-s} & =\sum_{d \mid 4 N} \mu(d) \psi(d) \sum_{m, n=1}^{\infty} \psi(m n)(d m)^{-(s-\lambda+1)} n^{\lambda}(u n)^{-s} \\
& =\sum_{d \mid 4 N} \mu(d) \psi(d) d^{\lambda-1} \sum_{m, n=1}^{\infty} \psi(m n)(m n)^{\lambda} m^{-1}(u d m n)^{-s}
\end{aligned}
$$


which easily gives the result. As for 5), the case $\lambda=1$ is obvious from 4). For $\lambda=0$, it is a kind of cheat, since there is no Shimura lift at this weight. We are hereby defining $S_{t}$ for weight $1 / 2$ by an extension of Theorem 2.17 to that weight:

$$
N_{t}(G)(w)=N_{t}(G)(i \infty)+C_{t}(0) S_{t}(G)(t w) \quad \text { with } C_{t}(0)=8(t N)^{1 / 4} .
$$

(Note. There is an extra factor of 2 in the case $\lambda=0$; see Theorem 2.12). Now 5) follows from Theorem 2.16 (and by verifying the integration). Finally, 6) is obvious from 2).

Corollary 3.2. Let

$$
E(w)=\sum_{d \mid 4 N} \mu(d) \psi(d) \sum_{m=1}^{\infty} \psi(m) \sigma(m) e(u d m w),
$$

with other notations as before. Then, for $\left(\begin{array}{ll}A & B \\ C & D\end{array}\right) \in S L(2, Z)$,

$$
\begin{aligned}
& \text { 1) }\left(\left.S_{t}(G)\right|_{2}\left(\begin{array}{ll}
A & B \\
C & D
\end{array}\right)\right)(i \infty)=\left(\left.E\right|_{2}\left(\begin{array}{ll}
A & B \\
C & D
\end{array}\right)\right)(i \infty) \quad \lambda=1 \\
& \text { 2) } \lim _{w \rightarrow i \infty}\left[\frac{1}{w}\left(\left.S_{t}(G)\right|_{0}\left(\begin{array}{ll}
A & B \\
C & D
\end{array}\right)\right)(w)\right]=2 \pi i u\left(\left.E\right|_{2}\left(\begin{array}{ll}
A & B \\
C & D
\end{array}\right)\right)(i \infty) \quad \lambda=0 .
\end{aligned}
$$

We shall now evaluate the RHS in Corollary 3.2 at various cusps. We begin by identifying the function $E$ as a linear combination of Eisenstein series.

Proposition 3.3. Let $E$ be as above, and, following Hecke [5], define the Eisenstein series

$$
G_{2}\left(z, a_{1}, a_{2}, r\right)=\lim _{s \rightarrow 0} \sum_{\substack{m_{1} \equiv a_{1}(r) \\ m_{2} \equiv a_{2}(r)}}^{\prime} \frac{1}{\left(m_{1}+z m_{2}\right)^{2}\left|m_{1}+z m_{2}\right|^{2 s}}
$$

(The indices $a_{1}, a_{2}$ are integers $(\bmod r)$, and $z \in H$; the RHS defines an analytic function for $\operatorname{Re}(s)>0$, which continues to the entire s-plane.) Then

$$
\begin{aligned}
E(w)= & \frac{-r^{2}}{8 \pi^{2} g(\bar{\psi})} \sum_{d \mid 4 N} \mu(d) \psi(d) \sum_{a_{1}=1}^{r} \sum_{a_{2}=1}^{r 2 u d} \psi\left(a_{2}\right) G_{2}\left(w, r u d a_{1}, a_{2}, r^{2} u d\right) \\
& +\frac{\psi(0) \phi(4 N)}{(4 \pi i)(4 N)(w-\bar{w})}
\end{aligned}
$$

(where $\phi$ is the Euler $\phi$-function.). 
Proof. Hecke derived the Fourier expansion

$$
\begin{aligned}
G_{2}\left(w, a_{1}, a_{2}, r\right)= & \frac{-2 \pi i}{r^{2}(w-\bar{w})}+\delta\left(\frac{a_{1}}{r}\right) \sum_{m_{2} \equiv a_{2}(r)}^{\prime} 1 / m_{2}^{2} \\
& -\frac{4 \pi^{2}}{r^{2}} \sum_{\substack{m m_{1}>0 \\
m_{1} \equiv a_{1}(r)}}|m| e\left(a_{2} m / r\right) e\left(m m_{1} w / r\right)
\end{aligned}
$$

where

$$
\delta(x)= \begin{cases}0 & x \notin Z \\ 1 & x \in Z .\end{cases}
$$

When $r=1$, the result (3.6) can be easily verified from (3.7). We assume now that $r>1$, so that $\psi$ is nontrivial. If we take a character sum

$$
\sum_{a_{1}, a_{2}=1}^{r} \psi\left(a_{1}\right) \overline{\psi\left(a_{2}\right)} G_{2}\left(w, a_{1}, a_{2}, r\right)
$$

it is clear that the first two terms on the RHS of (3.7) disappear; as for the third, we note that, since $\psi$ is primitive,

$$
\sum_{a_{2}=1}^{r} \overline{\psi\left(a_{2}\right)} e\left(a_{2} m / r\right)=g(\bar{\psi}) \psi(m)
$$

We thus find

$$
\begin{aligned}
\sum_{a_{1}, a_{2}=1}^{r} \psi\left(a_{1}\right) \overline{\psi\left(a_{2}\right)} G\left(w, a_{1}, a_{2}, r\right)=-\frac{4 \pi^{2}}{r^{2}} g(\bar{\psi}) \sum_{m m_{1}>0} \psi\left(m m_{1}\right)|m| e\left(m m_{1} w / r\right) \\
=-\frac{8 \pi^{2} g(\bar{\psi})}{r^{2}} \sum_{m=1}^{\infty} \sum_{m_{1}=1}^{\infty} \psi\left(m m_{1}\right) m e\left(m m_{1} w / r\right) \\
=-\frac{8 \pi^{2} g(\bar{\psi})}{r^{2}} \sum_{m=1}^{\infty} \psi(m) \sigma(m) e(m w / r) .
\end{aligned}
$$

Thus

$$
E(w)=\frac{-r^{2}}{8 \pi^{2} g(\bar{\psi})} \sum_{d \mid 4 N} \mu(d) \psi(d) \sum_{\alpha_{1}, a_{2}=1}^{r} \psi\left(a_{1}\right) \overline{\psi\left(a_{2}\right)} G_{2}\left(r u d w, a_{1}, a_{2}, r\right) .
$$

To complete the result, we need the following early verified identity: if $R$ is a positive integer, then

$$
G_{2}\left(R z, a_{1}, a_{2}, r\right)=\sum_{k=1}^{R} G_{2}\left(z, a_{1} R, a_{2}+k r, R r\right) .
$$

We now have 


$$
E(w)=\frac{-r^{2}}{8 \pi^{2} g(\bar{\psi})} \sum_{d \mid 4 N} \mu(d) \psi(d) \sum_{a_{1}, a_{2}=1}^{r} \psi\left(a_{1}\right) \overline{\psi\left(a_{2}\right)} \sum_{k=1}^{r u d} G_{2}\left(z, r u d a_{1}, a_{2}+k r, r^{2} u d\right)
$$

and this sum rearranges to give the stated result.

To establish the behavior of $E(w)$ at the cusps of $\Gamma_{0}(2 N)$, it suffices to examine $\left.E\right|_{2}\left(\begin{array}{ll}A & B \\ C & D\end{array}\right)$ at a set of $\Gamma_{0}(2 N)$-inequivalent cusps. We observe that every cusp $p / q$ is $\Gamma_{0}(2 N)$-equivalent to a cusp of the form $1 / C$. Therefore we examine $\left.E\right|_{2}\left(\begin{array}{ll}1 & 0 \\ C & 1\end{array}\right)$.

Theorem 3.4. Let $C \in Z$, and define $\alpha, \beta \in Z$ by $C / r u=\alpha / \beta$ with $(\alpha, \beta)$ = 1 . Then

$$
\left.E\right|_{2}\left(\begin{array}{ll}
1 & 0 \\
C & 1
\end{array}\right)(i \infty)=\left\{\begin{array}{l}
\frac{-r g(\psi)}{24} \frac{\psi(\alpha) \overline{\psi(\beta)}}{\beta^{2}} \prod_{p \mid 4 N}\left(1-p^{-2}\right) \text { if }(\alpha, 4 N)=1 \\
0 \text { otherwise. }
\end{array}\right.
$$

In particular, $E$ vanishes at any cusp $1 / C$ where $r \nmid C$.

Proof. We use two straightforward results for Eisenstein series:

$$
\left.G_{2}\right|_{2}\left(\begin{array}{ll}
A & B \\
C & D
\end{array}\right)=G_{2}\left(w, A a_{1}+C a_{2}, B a_{1}+D a_{2}, r\right)
$$

and

$$
G_{2}\left(i \infty, a_{1}, a_{2}, r\right)=\left\{\begin{array}{l}
0 \text { if } a_{1} \not \equiv 0(r) \\
\sum_{m_{2} \equiv a_{2}(r)}^{\prime} \frac{1}{m_{2}^{2}} \text { if } a_{1} \equiv 0(r) .
\end{array}\right.
$$

(The first is derived from (3.5), the second from (3.7).) From (3.6) we get

$$
\begin{aligned}
\left.E_{2}\right|_{2}\left(\begin{array}{ll}
1 & 0 \\
C & 1
\end{array}\right)(i \infty)= & \frac{-r^{2}}{8 \pi^{2} g(\bar{\psi})} \sum_{d \mid 4 N} \mu(d) \psi(d) \\
& \times \sum_{a_{1}=1}^{r} \sum_{a_{2}=1}^{r 2 u d} \psi\left(a_{1}\right) \overline{\psi\left(a_{2}\right)} G_{2}\left(i \infty, r u d a_{1}+C a_{2}, a_{2}, r^{2} u d\right) .
\end{aligned}
$$

Now by (3.12), only those terms with $r u d a_{1}+C a_{2} \equiv 0\left(r^{2} u d\right)$ will contribute, each giving $\sum_{m \equiv a_{2}\left(r^{2} u d\right)}^{\prime} 1 / m^{2}$. To select those terms we use the standard ruse

$$
\frac{1}{r^{2} u d} \sum_{k=1}^{r^{2} u d} e\left[\left(r u d a_{1}+C a_{2}\right) k / r^{2} u d\right]=\left\{\begin{array}{l}
0 \\
1
\end{array} .\right.
$$

With this extra factor in (3.13), the sum over $a_{1}$ is just 


$$
\sum_{a_{1}=1}^{r} \psi\left(a_{1}\right) e\left(a_{1} k / r\right)=g(\psi) \overline{\psi(k)}
$$

and so the sum over $k$ is just

$$
\begin{aligned}
\sum_{k=1}^{r 2 u d} \overline{\psi(k) e}\left(C a_{2} k / r^{2} u d\right) & =\sum_{h=1}^{r u d} \sum_{k=1}^{r} \overline{\psi(k)} e\left(C a_{2}(r h+k) / r^{2} u d\right) \\
& =\sum_{k=1}^{r} \overline{\psi(k)} e\left(C a_{2} k / r^{2} u d\right) \sum_{h=1}^{r u d} e\left(C a_{2} h / r u d\right) \\
& =g(\bar{\psi}) \psi\left(\frac{C a_{2}}{r u d}\right) r u d
\end{aligned}
$$

using the convention $\psi(a / b)=0$ if $b \nmid a$. Putting this back together gives

$$
\left.E\right|_{2}\left(\begin{array}{ll}
1 & 0 \\
C & 1
\end{array}\right)(i \infty)=\frac{-r g(\psi)}{8 \pi^{2}} \sum_{d \mid 4 N} \mu(d) \psi(d) \sum_{a_{2}=1}^{r 2 u d} \overline{\psi\left(a_{2}\right)} \psi\left(\frac{C a_{2}}{r u d}\right) \sum_{m \equiv a_{2}\left(r^{2} u d\right)}^{\prime} 1 / m^{2} .
$$

The statement of the theorem follows by a straightforward sequence of calculations. (But that $E$ vanishes when $r \nmid C$ is clear already from the term $\overline{\psi\left(a_{2}\right)} \psi\left(C a_{2} / r u d\right)$.)

Remarks. We have seen earlier that the Niwa lifting is not defined for $\lambda=0, \chi=1$, if $a(0) \neq 0$, because the integral is not absolutely convergent. Hewever, the lifting defined by Proposition 4.1 is perfectly respectable even in this case. One sees that $\theta(z)=\sum_{-\infty}^{\infty} e\left(n^{2} z\right)$ 'lifts' to

$$
-\sum_{d \mid 4 N} \frac{\mu(d)}{d} \log |\eta(d w)|
$$

where $\eta(w)=e(w / 24) \prod_{n=1}^{\infty}(1-e(n w))$ is the Dedekind eta function.

It is easy to see that this lifting transforms at level $2 N$. On differences of theta functions, everything agrees:

$$
\begin{aligned}
N_{1}\left(\theta(z)-\theta\left(u^{2} z\right)\right)(w) & =C_{1}(0) S_{1}\left(\theta(z)-\theta\left(u^{2} z\right)\right)(w) \\
& =8 N^{1 / 4} \sum_{d \mid 4 N} \frac{\mu(d)}{d} \log \left|\frac{\eta(u d z)}{\eta(d z)}\right| \text { if } u^{2} \mid N
\end{aligned}
$$

\section{$\S 4$, Behavior at the cusps}

In this section we consider the behavior of lifted forms at the cusps. We let $G \in S_{\kappa / 2}(4 N, \chi)$ for $\kappa \geq 3$, or $G \in G_{1 / 2}(4 N, \chi)$, and let

$$
\Phi(w)=\int_{\Gamma_{0}(4 N) \backslash H} v^{\kappa / 2} G(z) \overline{\theta^{*}} \overline{z, w)} d_{0} z .
$$


We shall show the following:

If $\kappa \geq 5, \Phi$ is a cusp form. (Shimura's result)

If $\kappa=3$, the value of $\Phi$ at a cusp is given as the inner product of $G$ against a linear combination of theta functions of weight $3 / 2$. From this will follow Shimura's conjecture, that the orthogonal complement to the space of theta functions always lifts to cusp forms.

If $\kappa=1$, the 'pole term' of $\Phi$ at a cusp is given as the inner product of $G$ against a linear combination of theta functions of weight $1 / 2$.

1. We being by stating a precise formulation of Shimura's conjecture. Let $f, g \in S_{\kappa / 2}(4 N, \chi)$. We say that $f$ and $g$ are orthogonal when $\int_{\Gamma_{0}(4 N) \backslash H}$ $v^{k / 2} f(z) \overline{g(z)} d_{0} z=0$. If $U$ is a subspace of $S_{k / 2}(4 N, \chi)$, we say that $f$ is orthogonal to $U$ if $f$ is orthogonal to every $g \in U$. We write " $\perp$ " for "orthogonal".

Let $t$ be a square-free positive integer, and define $U_{t}$ as the subspace of $S_{3 / 2}(4 N, \chi)$ spanned by functions of the form $h_{\psi}\left(t d^{2} z\right)$, with $d$ an integer. That is,

$$
U_{t}=\left\{h_{\psi}\left(t d^{2} z\right)\left|\quad 4 t d^{2} r^{2}\right| 4 N, \chi=\psi(-t)\right\}
$$

(See Section 3).

Recall that $S_{t}(G)=\sum_{n=1}^{\infty} A_{t}(n) e(n w)$, where $\sum_{n=1}^{\infty} A_{t}(n) n^{-s}=L\left(s, \chi_{t}\right)$ $\times \sum_{n=1}^{\infty} a\left(t n^{2}\right) / n^{s}$ with $\chi_{t}=\chi(-t /)$. Then Shimura's conjecture asserts

$$
S_{t}(G) \text { is a cusp form if and only if } G \perp U_{t} \text {. }
$$

In view of the relation $S_{t}(G)(w)=S_{1}\left(G_{t}\right)(w)\left(G_{t}(z)=G(t z)\right)$, it is not hard to show that Shimura's conjecture is true for all $t$ if and only if it is true for $t=1$. However, since we are after explicit formulae, it will be necessary to do the general case.

2. Because a character is easier to deal with than a Gaussian sum, we shall consider the behavior at cusps of the function

$$
\Psi(w)=\left.\Phi\right|_{2 \lambda} W(2 N)=\int_{\Gamma_{0}(4 N) \backslash H} v^{\kappa / 2} F(z) \theta(z, w) d_{0} z
$$

where $F=\left.G\right|_{k / 2} W(4 N)$. We shall study $\lim _{\eta \rightarrow \infty}\left(\left.\Psi\right|_{2 \lambda} \alpha^{-1}\right)(i \eta)$ for $\lambda>0$, and $\lim _{\eta \rightarrow \infty}\left(1 / i \eta\left(\left.\Psi\right|_{0} \alpha^{-1}\right)(i \eta)\right)$ for $\lambda=0$, for enough matrices $\alpha \in S L(2, Z)$ to establish the behavior of $\Psi$ (and thus $\Phi$ ) at all cusps of $\Gamma_{0}(2 N) \backslash H$. The following lemma will prove useful: 
Lemma 4.1. Suppose c contains every prime factor of $2 N$. Then, for $(c, d)=1$, the cusp $-d / c$ is $\Gamma_{0}(2 N)$-equivalent to $1 / c^{\prime}$ for some $c^{\prime}$ which also contains every prime factor of $2 N$.

Proof. Since $(c, d)=1$, let $\alpha, \beta$ solve $-d \alpha+c \beta=1$. Since $c$ contains all prime factors of $2 N,(\alpha, 2 N \beta)=1$. Let $\gamma, \delta$ solve $\alpha \delta-2 N \gamma \beta=1$. The $\operatorname{matrix}\left(\begin{array}{cc}\alpha & \beta \\ 2 N \gamma & \delta\end{array}\right) \in \Gamma_{0}(2 N)$ does the trick:

$$
\frac{\alpha(-d / c)+\beta}{2 N \gamma(-d / c)+\delta}=\frac{-d \alpha+c \beta}{-2 N \gamma d+c \delta}=\frac{1}{c^{\prime}}, \quad \text { where } c^{\prime}=c \delta-2 N \gamma d
$$

Remark. $\quad c^{\prime}$ contains precisely the same factor of $2 N$ as $c$.

From Proposition 2.4, we have the following:

$$
\left(\left.\Psi\right|_{2 \lambda} \alpha^{-1}\right)(w)=\int_{\Gamma_{0}(4 N) \backslash H} v^{\kappa / 2} F(z) \bar{\theta}_{\alpha}(z, \bar{w}) d_{0} z
$$

where

$$
\theta_{\alpha}(z, w)=(4 \eta)^{-\lambda} v^{1 / 2} \sum_{x \in L^{\prime}} \bar{\chi}_{1}\left(x_{1}\right) \Lambda(\hat{x}, w)^{\lambda} \exp \left[\frac{-4 \pi v}{N}|\Lambda(\hat{x}, w)|^{2}\right] e(\bar{z} D(x) / N)
$$

with

$$
\begin{gathered}
\Lambda(\hat{x}, w)=\frac{1}{\eta}\left(\frac{1}{4} \hat{x}_{1}-w \hat{x}_{2}+4 w^{2} \hat{x}_{3}\right), \quad D(\hat{x})=D(x)=x_{2}^{2}-4 x_{1} x_{3}, \\
L^{\prime}=Z \oplus N Z \oplus N Z / 4, \quad \text { and } \quad \hat{x}=\hat{\alpha} x
\end{gathered}
$$

where

$$
\hat{\alpha}=\left(\begin{array}{ccc}
a^{2} & 4 a b & 16 b^{2} \\
\frac{1}{2} a c & a d+b c & 8 b d \\
\frac{1}{16} c^{2} & \frac{1}{4} c d & d^{2}
\end{array}\right) \quad \text { when } \quad \alpha=\left(\begin{array}{ll}
a & b \\
c & d
\end{array}\right)
$$

The cusp in question here is the rational point $-d / c$. We shall study $\lim _{\eta \rightarrow \infty} \theta_{a}(z, i \eta)$ for $\lambda>0$ and $\lim _{\eta \rightarrow \infty}\left(-1 / i \eta \theta_{\alpha}(z, i \eta)\right)$ for $\lambda=0$. Of course arguments must be made for bringing the limit inside the integral. (A lot depends on $F$ being a cusp form for $k \geq 3$; for instance, although $\lim _{\eta \rightarrow \infty} \theta_{\alpha}(z, i \eta)=0$ for all $\alpha$ when $\lambda>1$, we have already seen that Eisenstein series lift to Eisenstein series, which are not cusp forms.) These 
arguments-which are largely tedious estimates-are isolated in Appendix B.

It will be convenient to have an 'inversion' formula for inner products against theta functions.

Proposition 4.2. Let $N=r^{2} p q$, and let $\chi_{1} \bmod 4 N$ be induced by the primitive character $\psi \bmod r$. Let $G \in G_{\kappa / 2}(4 N, \chi)$ and let $F=\left.G\right|_{\kappa / 2} W(4 N)$. Then

$$
\int_{\Gamma_{0}(4 N) \backslash H} v^{\kappa / 2} F(z) \overline{h_{\bar{\psi}}} \overline{(p z)} d_{0} z=\frac{(-i r)^{\alpha} q^{\kappa / 2}}{N^{\kappa / 4}} g(\psi) \int_{\Gamma_{0}(4 N) \backslash H} v^{\kappa / 2} G(z) \overline{h_{\psi}(q z)} d_{0} z
$$

Proof. This is a straightforward application of Theorem 1.10 (iii), part (4).

3. We begin with the case $t=1$.

From (4.4) it is easy to see that

$$
\begin{aligned}
\theta_{\alpha}(z, i \eta)= & (4 \eta)^{-\lambda} v^{1 / 2} \sum_{x \in L^{\prime}} \bar{x}_{1}\left(x_{1}\right)\left[\left(\frac{1}{4 \eta} \hat{x}_{1}-4 \eta \hat{x}_{3}\right)-i \hat{x}_{2}\right]^{2} \\
& \times \exp \left[-\frac{8 \pi i}{N} u \hat{x}_{1} \hat{x}_{3}-\frac{4 \pi v}{N}\left(\frac{1}{16 \eta^{2}} \hat{x}_{1}^{2}+16 \eta^{2} \hat{x}_{3}^{2}\right)\right] e\left(z \hat{x}_{2}^{2} / N\right) .
\end{aligned}
$$

Because of the $\eta^{2} \hat{x}_{3}^{2}$ in the exponential, one expects those terms with $\hat{x}_{3}$ $\neq 0$ to contribute negligibly to the sum. The analysis of Appendix B shows this to be the case. We have

$$
\theta_{\alpha}(z, i \eta)=(4 \eta)^{-\lambda} v^{1 / 2} \sum_{\substack{x \in L^{\prime} \\ \hat{x}_{3}=0}} \bar{\chi}_{1}\left(x_{1}\right)\left(\frac{1}{4 \eta} \hat{x}_{1}-i \hat{x}_{2}\right)^{\lambda} \exp \left[-\frac{\pi v}{4 N \eta^{2}} \hat{x}_{1}^{2}\right] e\left(z \hat{x}_{2}^{2} / N\right)+\varepsilon
$$

where $\varepsilon=\varepsilon(z, \eta)$ is such that

$$
\int_{\Gamma_{0}(4 N) \backslash H} v^{k / 2} F(z) \varepsilon \overline{(z, \eta)} d_{0} z=\left\{\begin{array}{ll}
o(1) & \lambda>0 \\
O(1) & \lambda=0
\end{array} \quad \eta \rightarrow \infty .\right.
$$

The same analysis shows that, for non-negligibility, the sum may have at most one power of $\eta$ in the denominator of its polynomial term. (The inversion which brings the $\eta^{2}$ to the numerator of the exponential takes up exactly one power of $\eta$; anything more will cause the sum to vanish as $\eta \rightarrow \infty$.) Thus we see immediately that $\Psi$ is a cusp form as soon as $\lambda>1-i . e$. , for $\kappa \geq 5$ :

ThEOREM 4.3. For $\kappa \geq 5$, and any square-free $t, S_{t}(G) \in S_{22}\left(2 N, \chi^{2}\right)$. 
Proof. For $t=1$, we have just proved it. For other $t$, we cite the relation $S_{t}(G)(t w)=S_{1}\left(G_{t}\right)(w)$, where $G_{t}(z)=G(t z) \in S_{\kappa / 2}\left(4 N t, \chi_{t}\right)$.

In the remainder of this section and the next, we continue only with the cases $\lambda=0,1$ (weights $1 / 2$ and $3 / 2$ ). Since we want the pole term for $\lambda=0$, and the constant term for $\lambda=1$. we study

$$
\lim _{\eta \rightarrow \infty}\left[(i \eta)^{\lambda-1} \theta_{\alpha}(z, i \eta)\right] \text {. }
$$

For this function, the term $(1 / 4 \eta) \hat{x}_{1}$ in (4.6) is seen to be negligible for the same reasons as above, so that

$$
(-i \eta)^{\lambda-1} \theta_{\alpha}(z, i \eta)=(-1)^{\lambda} \frac{i v^{1 / 2}}{4^{\lambda} \eta} \sum_{\hat{x}_{3}=0} \bar{\chi}_{1}\left(x_{1}\right) \hat{x}_{2}^{\lambda} \exp \left(-\frac{\pi v}{4 N \eta^{2}} \hat{x}_{1}^{2}\right) e\left(z \hat{x}_{2}^{2} / N\right)+\varepsilon .
$$

The condition $\hat{x}_{3}=0$ has important consequences which we now explore.

By definition, $\hat{x}_{3}=0$ if and only if $c^{2} x_{1}+4 c d x_{2}+16 d^{2} x_{3}=0$. Let $x_{2}$ $=N y_{2}, x_{3}=N y_{3} / 4$, so that $y_{2}, y_{3} \in Z$. Then $\hat{x}_{3}=0$ if and only if $c^{2} x_{1}+4 N c d y_{2}$ $+4 N d^{2} y_{3}=0$. Now if $4 N \nmid c^{2}$, then $\left(x_{1}, 4 N\right)>1$, in which case $\chi_{1}\left(x_{1}\right)=0$, so that $(i \eta)^{\lambda-1} \theta_{\alpha}(z, i \eta)=\varepsilon$. That is, for $\lambda=1(\lambda=0)$,

$\Psi$ vanishes (has no pole) at any cusp $-d / c(c, d)=1$, where $4 N \nmid c^{2}$.

Assume now that $4 N \mid c^{2}$. Writing $4 N=4 \mu^{2} \tau$ with $\tau$ square-free, we must have $c=2 \mu \tau c_{1}$ for some $c_{1}$, and thus, by Lemma 4.1, we may assume $d=-1$. We see immediately

$$
\hat{x}_{3}=0 \text { if and only if } \tau c_{1}^{2} x_{1}-2 \mu \tau c_{1} y_{2}+y_{3}=0 .
$$

Clearly the RHS has a solution $y_{3}$ for any choice of $x_{1}, y_{2}$. As a convenience, let us also write $x_{1}=d y_{1}=-y_{1}$. Now

$$
\hat{\alpha}^{-1}=\widehat{\alpha^{-1}}=\left(\begin{array}{ccc}
d^{2} & -4 b d & 16 b^{2} \\
-\frac{1}{2} c d & a d+b c & -8 a b \\
\frac{1}{16} c^{2} & -\frac{1}{4} a c & a^{2}
\end{array}\right) \text {. }
$$

Hence, when $\hat{x}_{3}=0$, we have

$$
\left(\begin{array}{l}
x_{1} \\
x_{2}
\end{array}\right)=\left(\begin{array}{cc}
d^{2} & -4 b d \\
-\frac{1}{2} c d & a d+b c
\end{array}\right)\left(\begin{array}{l}
\hat{x}_{1} \\
\hat{x}_{2}
\end{array}\right) .
$$


In the present case $(d=-1)$ this inverts to give

$$
\begin{aligned}
& \hat{x}_{1}=(a-b c) y_{1}-4 N b y_{2} \\
& \hat{x}_{2}=\mu \tau c_{1} y_{1}+\mu^{2} \tau y_{2} .
\end{aligned}
$$

We see immediately that $\bar{\chi}_{1}\left(x_{1}\right)=\bar{\chi}_{1}\left(-y_{1}\right)=(-1)^{2} \chi_{1}(a-b c) \bar{\chi}_{1}\left(\hat{x}_{1}\right)$. Let us factor $\mu=m n$ so that $c_{1}=m c_{2}$ with $\left(c_{2}, n\right)=1$. This is clearly unique, given $c_{1}$; also, conversely, given a factorization $\mu=m n$, there is (more than one) $c_{1}$ such that $c_{1}=m c_{2}$ with $\left(c_{2}, n\right)=1$. Then

$$
\hat{x}_{2}=m^{2} n \tau\left(c_{2} y_{1}+n y_{2}\right) \quad y_{1}, y_{2} \in Z \text {. }
$$

Fix $\bar{y}_{1}, \bar{y}_{2}$ as a solution to $c_{2} y_{1}+n y_{2}=1$, and set

$$
\mathrm{g}=(a-b c) \bar{y}_{1}-4 N b \bar{y}_{2} .
$$

Let $\hat{x}_{1}(k)=k g$ for $k \in Z$. Now $\hat{x}_{1}(k)$ is a value taken on by $\hat{x}_{1}$ when $\hat{x}_{2}$ takes the value $m^{2} n \tau k$. If $\hat{x}_{1}^{\prime}(k)$ is another such value for $\hat{x}_{1}$, a simple argument shows that $\hat{x}_{1}^{\prime}(k)=\hat{x}_{1}(k)+p n$ for some integer $p$. Furthermore, each integer $p$ gives such a value. Thus when $\hat{x}_{2}=m^{2} n \tau k, \hat{x}_{1}$ runs over all integers congruent to $\mathrm{kg}$ modulo $n$. We may now write

$$
\begin{aligned}
&(-i \eta)^{\lambda-1} \theta_{\alpha}(z, i \eta)=(-1)^{\lambda} \frac{i v^{1 / 2}}{4^{\lambda} \eta}(-1)^{\lambda} \chi_{1}(a-b c) \\
& \times \sum_{k=-\infty}^{\infty} \sum_{h \equiv k g(n)} \bar{\chi}_{1}(h)\left(m^{2} n \tau k\right)^{\lambda} \exp \left(-\frac{\pi v h^{2}}{4 N \eta^{2}}\right) . \\
& e\left(z\left(m^{2} n \tau k\right)^{2} / N\right)+\varepsilon \\
&=i\left(\frac{m^{2} n \tau}{4}\right)^{\lambda} \chi_{1}(a-b c) \sum_{k \equiv-\infty}^{\infty}\left(\frac{v^{1 / 2}}{\eta} \sum_{h \equiv k g(n)} \bar{\chi}_{1}(h) \exp \left(-\frac{\pi v h^{2}}{4 N \eta^{2}}\right)\right) \\
& \times k^{\lambda} e\left(m^{2} \tau k^{2} z\right)+\varepsilon .
\end{aligned}
$$

Consider for the moment the inner sum

$$
\begin{aligned}
& \frac{v^{1 / 2}}{\eta} \sum_{h \equiv k g(n)} \bar{\chi}_{1}(h) \exp \left(-\frac{\pi v h^{2}}{4 N \eta^{2}}\right) \\
& =\frac{v^{1 / 2}}{\eta} \sum_{q=1}^{4 m 2 n \tau} \bar{\chi}_{1}(k g+n q) \sum_{h=k g+n q(4 N)} e\left(h^{2} i v / 8 N \eta^{2}\right) \\
& =\frac{v^{1 / 2}}{n \eta} \sum_{q=1}^{4 N} \bar{\chi}_{1}(k g+n q) \sum_{h \equiv k g+n q(4 N)} e\left(h^{2} i v / 8 N \eta^{2}\right) .
\end{aligned}
$$

Now concentrate on the sum 


$$
\begin{aligned}
\frac{v^{1 / 2}}{\eta} & \sum_{h \equiv K(4 N)} e\left(h^{2} i v / 8 N \eta^{2}\right) \quad(K=k g+n q) \\
& =(-8 N i Z)^{-1 / 2} \sum_{h \equiv K(4 N)} e\left(-h^{2} / 4(4 N)^{2} Z\right) \quad\left(Z=i \eta^{2} / 8 N v\right) \\
& =(4 N)^{-1 / 2}(-2 i Z)^{-1 / 2} \theta\left(-1 / 4(4 N)^{2} Z, K, 4 N\right) \\
& =(4 N)^{-1 / 2} \sum_{H=1}^{4 N} e(H K / 4 N) \theta(Z, H, 4 N)
\end{aligned}
$$

using the theta series of Theorem 1.10 (i). From the estimates of that theorem, we see this equals

$$
(4 N)^{-1 / 2}+O\left(v^{1 / 2} / \eta\right)
$$

The error again disappears with $\eta \rightarrow \infty$, when integrated against a form $F$ which is bounded at the cusps. Thus

$$
\begin{aligned}
(-i \eta)^{\lambda-1} \theta_{\alpha}(z, i \eta)= & \frac{i}{n(4 N)^{1 / 2}}\left(\frac{m^{2} n \tau}{4}\right)^{\lambda} \chi_{1}(a-b c) \\
& \times \sum_{k=-\infty}^{\infty}\left(\sum_{q=1}^{4 N} \bar{\chi}_{1}(k g+q n)\right) k^{\lambda} e\left(k^{2} m^{2} \tau z\right)+\varepsilon .
\end{aligned}
$$

Now $g=(a-b c) \bar{y}_{1}-4 N b \bar{y}_{2}$ with $1=c_{2} \bar{y}_{1}+n \bar{y}_{2}$. Therefore $\bar{\chi}_{1}(k g+n q)=$ $\bar{\chi}_{1}\left(k(a-b c) \bar{y}_{1}+n q\right)$. Since $(a-b c, N)=1$, we may factor $\bar{\chi}_{1}(a-b c)$ out of the character sum. This leaves

$$
(-i \eta)^{2-1} \theta_{\alpha}(z, i \eta)=\frac{i}{n(4 N)^{1 / 2}}\left(\frac{m^{2} n \tau}{4}\right)^{\lambda} \sum_{k=-\infty}^{\infty}\left(\sum_{q=1}^{4 N} \bar{\chi}_{1}\left(k \bar{y}_{1}+n q\right)\right) k^{\lambda} e\left(k^{2} m^{2} \tau z\right)+\varepsilon
$$

where $\alpha(1 / c)=i \infty, c=2 m^{2} n \tau c_{2}$, and $c_{2} \bar{y}_{1}+n \bar{y}_{2}=1$. Thus, at the cusp $1 / c$ with $c=2 m^{2} n \tau c_{2},\left(c_{2}, n\right)=1$, the constant (pole) term of $\Psi$ is

$$
\left((i \eta)^{\lambda-1}\left(\left.\Psi\right|_{2 \lambda} \alpha^{-1}\right)\right)(i \infty)=\frac{-i}{n(4 N)^{1 / 2}}\left(\frac{m^{2} n \tau}{4}\right)^{\lambda} \int_{\Gamma_{0}(4 N) \backslash H} v^{\kappa / 2} F(z) \overline{H_{\lambda}\left(m^{2} \tau z\right)} d_{0} z
$$

where

$$
H_{\lambda}(z)=\sum_{k=-\infty}^{\infty}\left(\sum_{q=1}^{4 N} \bar{\chi}_{1}\left(k \bar{y}_{1}+q n\right)\right) k^{\lambda} e\left(k^{2} z\right), \quad c_{2} \bar{y}_{1} \equiv 1(n) .
$$

Now suppose $\chi_{1} \bmod 4 N$ is induced by the primitive character $\psi \bmod$ $r$. If $r \nmid n$, then $H(z)=0$, because, as is shown in Appendix A, the inside character sum is zero. In particular, if $r^{2} \nmid N$, then $\Psi$ is a cusp form (is bounded at all cusps). 
On the other hand, if $r \mid n$, then $(1 / Q) \sum_{q=1}^{4 N} \bar{\chi}_{1}(k+q n)$ is a character $\bmod n$, induced by $\bar{\psi}$, where $Q=\sum_{q=1}^{4 N} \bar{\chi}_{1}(1+q n)$. We have

$$
H_{\lambda}(z)=2 Q \psi\left(c_{2}\right) \sum_{d \mid n} \mu(d) \bar{\psi}(d) d^{\lambda} h_{\bar{\psi}}\left(d^{2} z\right)
$$

where $h_{\psi}(z)=1 / 2 \sum_{k=-\infty}^{\infty} \psi(k) k^{\lambda} e\left(k^{2} z\right)$. The constant (pole) term of $\psi$ at $1 / c$ is thus

$$
\begin{array}{r}
\frac{-i Q \bar{\psi}\left(c_{2}\right)}{n N^{1 / 2}}\left(\frac{m^{2} n \tau}{4}\right)^{\lambda} \sum_{d \mid n} \mu(d) \psi(d) d^{\lambda} \int_{\Gamma_{0}(4 N) \backslash H} v^{\kappa / 2} F(z) h_{\bar{\psi}}\left(m^{2} \tau d^{2} z\right) d_{0} z \\
\left.=\frac{-i Q \bar{\psi}\left(c_{2}\right)\left(-i m^{2} n r \tau\right.}{n N^{3 / 4}}\right)^{\lambda} g(\psi) \sum_{n=r d \tilde{\delta}} \mu(d) \psi(d) d^{\lambda} \delta^{2 \lambda+1} \\
\quad \times \int_{\Gamma_{0}(4 N) \backslash H} v^{\kappa / 2} G(z) h_{\psi}\left(\delta^{2} z\right) d_{0} z
\end{array}
$$

using the inversion formula (4.5). This can be simplified to equal

$$
\frac{Q \bar{\psi}\left(c_{2}\right) g(\psi)}{4^{\lambda} r}(-i)^{\lambda+1} N^{\lambda / 2-3 / 4} \sum_{n=r d \delta} \mu(d) \psi(d) \delta^{\lambda} / d \int_{\Gamma_{0}(4 N) \backslash H} v^{\kappa / 2} G(z) h_{\psi}\left(\delta^{2} z\right) d_{0} z .
$$

We summarize these results in the following theorem:

THEOREM 4.4. Let $N=m^{2} n^{2} \tau$ be some factorization of $N$, with $\tau$ squarefree, and let $c=2 m^{2} n \tau c_{2}$ with $\left(c_{2}, n\right)=1$. Suppose $\chi_{1} \bmod 4 N$ is induced by the primitive character $\psi \bmod r$, and let $Q=\sum_{q=1}^{4 N} \bar{\chi}_{1}(1+q n)$. Then, for $\lambda=1(\lambda=0)$,

(1) $\Psi$ automatically vanishes (has no pole) at all cusps not of the form $-d / c$ with $c$ as above

(2) Every cusp - $d / c^{\prime}$ with $c^{\prime}$ as above is $\Gamma_{0}(2 N)$-equivalent to a cusp of the form $1 / c$ with $c$ as above. $\Psi$ has similar behavior at equivalent cusps.

(3) At the cusp $1 / c$, the constant (pole) term of $\Psi$ is

$$
\left(\left.(i \eta)^{\lambda-1} \Psi\right|_{2 \lambda}\left(\begin{array}{ll}
1 & 0 \\
c & 1
\end{array}\right)\right)(i \infty)=\frac{-i}{n(4 N)^{1 / 2}}\left(\frac{m^{2} n \tau}{4}\right)^{\lambda} \int_{\Gamma_{0}(4 N) \backslash H} v^{\kappa / 2} F(z) H_{\lambda}\left(m^{2} \tau z\right) d z
$$

with

$$
H_{\lambda}(z)=\sum_{k=-\infty}^{\infty}\left(\sum_{q=1}^{4 N} \bar{\chi}_{1}\left(k \bar{y}_{1}+q n\right)\right) k^{\lambda} e\left(k^{2} z\right), \quad c_{2} \bar{y}_{1} \equiv 1(n) .
$$

(4) If $r \nmid n$, then $\Psi$ vanishes (has no pole) at $1 / c$. In particular, if $r^{2} \nmid N$, then $\Psi$ is a cusp form (is bounded).

(5) If $r \mid n$, then the constant (pole) term of $\Psi$ at $1 / c$ is 


$$
\frac{Q \bar{\psi}\left(c_{2}\right) g(\psi)}{4^{\lambda} r}(-i)^{\lambda+1} N^{\lambda / 2-3 / 4} \sum_{n=r d \delta} \mu(d) \psi(d) \delta^{\lambda} / d \int_{\Gamma_{0}(4 N) \backslash H} v^{\kappa / 2} G(z) \overline{h_{\psi}} \overline{\left(\delta^{2} z\right)} d_{0} z .
$$

Corollary 4.5. Let $G \in S_{3 / 2}(4 N, \chi)$. Then $S_{1}(G)$ is a cusp form if and only if $G \perp U_{1}$.

Proof. One direction is clear from the theorem. It remains to prove the converse: if $\Psi$ is a cusp form, then $G(z)$ is orthogonal to all $h_{\psi}\left(\delta^{2} z\right)$ with $\delta^{2} r^{2} \mid N$. To show this, assume $\delta^{2} r^{2} \mid N$ and let $n=\delta r$. At the cusp $1 / 2 m^{2} n \tau$, we have, by (4.17)

$$
0=\sum_{d d^{\prime}=\delta} \mu(d) \psi(d) d^{\prime 2} \int_{\Gamma_{0}(4 N) \backslash H} v^{3 / 2} G(z) h_{\psi}\left(d^{\prime 2} z\right) d_{0} z
$$

Since $\psi(1)=\mu(1)=1$, we have

$$
\int_{\Gamma_{0}(4 N) \backslash H} v^{3 / 2} G(z) \overline{h_{\psi}}\left(\overline{\left.\delta^{2} z\right)} d_{0} z=\frac{-1}{\delta^{2}} \sum_{\substack{d d^{\prime}=\delta \\ d>1}} \mu(d) \psi(d) d^{\prime 2} \int_{\Gamma_{0}(4 N) \backslash H} v^{3 / 2} G(z) \overline{h_{\psi}\left(d^{\prime 2} z\right)} d_{0} z .\right.
$$

Now every $d^{\prime}$ on the RHS is smaller than $\delta$. By induction the RHS vanishes, the initial case $\delta=1$ obviously vanishing. Hence the LHS is zero.

4. Let now $t$ be a square-free positive integer. Recall the notation of Theorem 2.17:

$$
N_{t}(G)(w)=\int_{\Gamma_{0}(4 N t) \backslash H} v^{\kappa / 2} G(t z) \overline{\theta_{t}^{*}(z, w)} d_{0} z,
$$

where $\theta_{t}^{*}$ is formed from $\theta^{*}$ by replacing every $N$ by $N t$, and $\chi_{1}$ by $\chi_{t}=$ $\chi_{1}(t /)$. Recall that $N_{t}(G)(w)=C_{t}(\lambda) S_{t}(G)(t w)$, where $C_{t}(\lambda)=(-1)^{\lambda} 2^{-3 \lambda+2}$ $\cdot(N t)^{\lambda / 2+1 / 4}$ for $\lambda>0$ and $C_{t}(0)=8(N t)^{1 / 4}$. We now define

$$
\Psi_{t}(w)=\left.N_{t}(G)\right|_{2 \lambda} W(2 N t)(w) .
$$

On the one hand, $\Psi_{t}(w)=\left.C_{t}(\lambda) t^{-\lambda} S_{t}(G)\right|_{2 \lambda} W(2 N)(w)$, while on the other hand,

$$
\begin{aligned}
\Psi_{t}(w) & =\int_{\Gamma_{0}(4 N t) \backslash H} v^{\kappa / 2}\left(\left.G(t z)\right|_{\kappa / 2} W(4 N t)\right) \overline{\left(\left.\left.\theta_{t}^{*}\right|_{\kappa / 2} W(4 N t)\right|_{2 \lambda} \overline{W(2 N t))(z, w)}\right.} d_{0} z \\
& =\int_{\Gamma_{0}(4 N t) \backslash H} v^{\kappa / 2}\left(t^{-\kappa / 4} F(z)\right) \theta^{t}(\bar{z}, w) d_{0} z
\end{aligned}
$$

where $F=\left.G\right|_{\kappa / 2} W(4 N)$ is as before, and $\theta^{t}$ is $\theta$ with every $N$ replaced by $N t$, and $\chi_{1}$ replaced by $\chi_{t}$ :

$$
\theta^{t}(z, w)=(4 \eta)^{-\lambda} v^{1 / 2} \sum_{L_{t}} \bar{\chi}_{t}\left(x_{1}\right) \Lambda(x, w)^{\lambda} \exp \left(\frac{-4 \pi v}{N t}|\Lambda(x, w)|^{2}\right) e(\bar{z} D(x) / N t)
$$


with $\chi_{t}=\chi_{1}(t /), L_{t}=Z \oplus N t Z \oplus N t Z / 4$, and $\Lambda$ and $D$ as before. Since $\theta^{t}$ is a purely formal change from $\theta$, we may use directly the results of the previous section:

Proposition 4.6. Let $N t=m^{2} n^{2} \tau$ be some factorization of $N$, with $\tau$ square-free, and let $c=2 m^{2} n^{2} \tau c_{2}$ with $\left(c_{2}, n\right)=1$. Suppose $\chi_{t} \bmod 4 N t$ is induced by the primitive character $\psi \bmod r$. Then for $\lambda=1(\lambda=0)$,

(1) $\Psi_{t}$ vanishes (is bounded) at all cusps not of the form $-d / c$ with c as above.

(2) Every cusp - $d / c^{\prime}$ with $c^{\prime}$ as above is $\Gamma_{0}(2 N t)$-equivalent (and therefore $\Gamma_{0}(2 N)$-equivalent) to a cusp of the form $1 / c$ with $c$ as above. $\Psi$ has similar behavior at equivalent cusps.

(3) At the cusp $1 / 2 m^{2} n \tau c_{2},\left(c_{2}, n\right)=1$, the constant (pole) term of $\Psi_{t}$ is

$$
\left(\left.(i \eta)^{\lambda-1} \Psi_{t}\right|_{2 \lambda}\left(\begin{array}{ll}
1 & 0 \\
c & 1
\end{array}\right)\right)(i \infty)=\frac{-i t^{-\kappa / 4}}{n(4 N t)^{1 / 2}}\left(\frac{m^{2} n \tau}{4}\right)^{\lambda} \int_{\Gamma_{0}(4 N t) \backslash H} v^{\kappa / 2} F(z) H_{\lambda}\left(m^{2} \tau z\right) d_{0} z
$$

with

$$
H_{\lambda}(z)=\sum_{k=-\infty}^{\infty}\left(\sum_{q=1}^{4 N t} \bar{\chi}_{t}\left(k \bar{y}_{1}+q n\right)\right) k^{\lambda} e\left(k^{2} z\right), \quad c_{2} \bar{y}_{1} \equiv 1(n) .
$$

(4) If $r \nmid n$, then $\Psi$ vanishes (has no pole) at $1 / c$. In particular, if $r^{2} \nmid N t$, then $\Psi_{t}$ is a cusp form (is bounded).

Now the last statement really says something new, because if $t$ contains a prime not already in $4 N$, then $r$ also contains that prime, since $r$ is the conductor of $\chi_{t}$, which consists in part of the Legendre symbol $(p /)$ for any $p \mid t$. In that event, since $t$ is square-free $r^{2} \nmid N t$, and $\Psi_{t}$ is a cusp form (bounded form) by the proposition. Also, if $2 \nmid N$ but $2 \mid t$, then $8 \mid r$ and $r^{2} \nmid N t$, so that $\Psi_{t}$ is again a cusp (bounded) form. We have proved:

Theorem 4.7. Let $G \in S_{3 / 2}(4 N, \chi)$. If $t \nmid N$, then $S_{t}(G)$ is a cusp form.

Corollary 4.8. Let $G \in S_{3 / 2}(4 N, \chi)$. Suppose $t \nmid N$. Then $S_{t}(G)$ is a cusp form if and only if $G \perp U_{t}$.

Proof. $U_{t}$ is empty.

Remark. Similar statements can be made for $S_{t}(G)$ being bounded, when $G \in G_{1 / 2}(4 N, \chi)$.

In the remainder, we shall assume that $t \mid N$ and $r \mid n$. Let $Q=\sum_{q=1}^{4 N t}$ 
$\bar{\chi}_{t}(1+q n)$. Then $1 / Q \sum_{q=1}^{4 N t} \bar{\chi}_{t}(k+q n)$ is a character $\bmod n$, induced by the primitive character $\bar{\psi} \bmod r$. Then

$$
H_{\lambda}(z)=2 Q \psi\left(c_{2}\right) \sum_{d \mid n} \mu(d) \bar{\psi}(d) d^{\lambda} h_{\bar{\psi}}\left(d^{2} z\right) .
$$

The constant (pole) term of $\Psi_{t}$ at $1 / c$ is thus

$$
\frac{-i Q t^{-\kappa / 4} \bar{\psi}\left(c_{2}\right)}{n(N t)^{1 / 2}}\left(\frac{m^{2} n \tau}{4}\right)^{\lambda} \sum_{d \mid n} \mu(d) \psi(d) d^{\lambda} \int_{\Gamma_{0}(4 N t) \backslash H} v^{\kappa / 2} F(z) \overline{h_{\bar{\psi}}}\left(m^{2} \tau d^{2} z\right) d_{0} z .
$$

Our immediate goal is to "unfold" $\Gamma_{0}(4 N t) \backslash H$ back to $\Gamma_{0}(4 N) \backslash H$. Towards this we use the disjoint coset decomposition

$$
\Gamma_{0}(4 N)=\bigcup_{j=1}^{t} \Gamma_{0}(4 N t) \gamma_{j} \quad \gamma_{j}=\left(\begin{array}{cc}
1 & 0 \\
4 N j & 1
\end{array}\right) .
$$

(This relies on our assumption $t \mid N$.) Thus

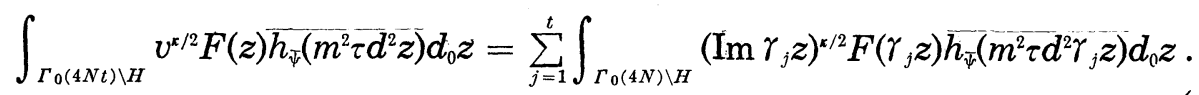

Now $\left(\operatorname{Im} \gamma_{j} z\right)^{\kappa / 2}=v^{\kappa / 2} /|4 N j z+1|^{\kappa}$, and $F\left(\gamma_{j} z\right)=(4 N j z+1)^{\kappa / 2} F(z)$. The hard work comes in reducing $h_{\bar{\psi}}$ :

$$
\begin{aligned}
h_{\bar{\psi}}\left(m^{2} \tau d^{2} \gamma_{j} z\right) & =h_{\vec{\psi}}\left(m^{2} \tau d^{2} z / 4 N j z+1\right) \\
& =h_{\bar{\psi}}\left(t m^{2} \tau d^{2}(z / t) / 4 m^{2} r^{2} d^{2} \delta^{2} \tau j(z / t)+1\right)
\end{aligned}
$$

writing $N t=m^{2} n^{2} \tau$ with $n=r d \delta$

$$
=h_{\bar{\psi}}\left(t Z / 4 r^{2} \delta^{2} j Z+1\right) \quad Z=m^{2} \tau d^{2} z / t .
$$

Since $t$ is square-free, we may write $t=t_{0} t_{1}$, with $t_{1} \mid \delta$ and $\left(t_{0}, \delta\right)=1$. Let $\delta=t_{1} \delta_{1}$, and continue the equality:

$$
\begin{aligned}
& =h_{\bar{\psi}}\left(t_{0} Z_{0} / 4 r^{2} t_{1} \delta_{1}^{2} j Z_{0}+1\right) \quad Z_{0}=m^{2} \tau d^{2} z / t_{0} \\
& =(4 N j z+1)^{x / 2}(-1)^{\lambda} r^{-1} t_{0}^{-\lambda-1} 2^{-1} \sum_{k=-\infty}^{\infty} k^{\lambda} \xi_{j}(k) e\left(k^{2} Z_{0} / t_{0}\right)
\end{aligned}
$$

using Theorem 1.10 (iii) part (5), where

$$
\xi_{j}(k)=\sum_{h=1}^{r} \sum_{g=1}^{t_{0} r} \bar{\psi}(h) e\left(\left(g k+t_{0} g h-r t_{1} \delta_{1}^{2} j g^{2}\right) / t_{0} r\right) .
$$

Altogether we have

$$
\begin{aligned}
& \int_{\Gamma_{0}(4 N t) \backslash H} v^{\kappa / 2} F(z){\overline{h_{\bar{\psi}}\left(m^{2} \tau d^{2} z\right) d_{0}}} z \\
& \quad=\frac{(-1)^{2}}{2 r t_{0}^{\lambda+1}} \int_{\Gamma_{0}(4 N) \backslash H} v^{\kappa / 2} F(z) \overline{\sum_{k=-\infty}^{\infty}\left(\sum_{j=1}^{t} \xi_{j}(k)\right) k^{\lambda} e\left(k^{2} Z_{0} / t_{0}\right)} d_{0} z .
\end{aligned}
$$


We now consider the sum over $j$ :

$$
\begin{aligned}
\sum_{j=1}^{t} \xi_{j}(k) & =\sum_{h=1}^{r} \sum_{g=1}^{t_{0} r} \sum_{j=1}^{t_{0} t_{1}} \bar{\psi}(h) e\left(\left(g k+t_{0} g h-u g^{2}\right)_{1} t_{0} r\right) \quad\left(u=r t_{i} \delta_{1}^{2} j\right) \\
& =\sum_{h=1}^{r} \sum_{g=1}^{t_{0} r} \bar{\psi}(h) e\left(\left(g k+t_{0} g h\right) / t_{0} r\right) \sum_{j=1}^{t_{0} t_{1}} e\left(-t_{1} \delta_{1}^{2} g^{2} j / t_{0}\right) .
\end{aligned}
$$

Since $\left(t_{0}, \delta\right)=1$, the last sum is nonzero only when $t_{0} \mid g^{2}$. Since $t$ is squarefree, this is equivalent to $t_{0} \mid g$, in which case the sum gives a factor of $t$ :

$$
\begin{aligned}
\sum_{j=1}^{t} \xi_{j}(k) & =t \sum_{h=1}^{r} \sum_{g=1}^{r} \bar{\psi}(h) e\left(\left(g k+t_{0} g h\right) / r\right) \\
& =\operatorname{tg}(\bar{\psi}) \sum_{g=1}^{r} \psi\left(g t_{0}\right) e(g k / r)=(-1)^{\lambda} r t \psi\left(t_{0}\right) \bar{\psi}(k)
\end{aligned}
$$

using $g(\bar{\psi}) g(\psi)=(-1)^{\lambda} r$. We now have

$$
\int_{\Gamma_{0}(4 N t) \backslash H} v^{\kappa / 2} F(z) \overline{h_{\Psi}} \overline{\left(m^{2} \tau d^{2} z\right)} d_{0} z=\bar{\psi}\left(t_{0}\right) \frac{t_{1}}{t_{0}^{\lambda}} \int_{\Gamma_{0}(4 N) \backslash H} v^{\kappa / 2} F(z) \overline{h_{\Psi}\left(\frac{m^{2} \tau d^{2}}{t_{0}^{2}} z\right)} d_{0} z .
$$

If $\left(t_{0}, r\right)>1, \bar{\psi}\left(t_{0}\right)=0$, and we needn't bother with the integral. Assume now $\left(t_{0}, r\right)=1$. Then, since $\left(t_{0}, \delta\right)=1$ also, the factor $m^{2} \tau d^{2} / t_{0}^{2}$ is an integer, and we have a factorization

$$
N=\frac{m^{2} n^{2} \tau}{t}=\frac{m^{2} r^{2} d^{2} \delta^{2} \tau}{t_{0} t_{1}}=\left(\frac{m^{2} \tau d^{2}}{t_{0}^{2}}\right)\left(\frac{\delta^{2} t_{0} t_{1}}{t_{1}^{2}}\right) r^{2}=\left(\frac{m^{2} \tau d^{2}}{t_{0}^{2}}\right)\left(t \delta_{1}^{2}\right) r^{2}
$$

The inversion formula (4.5) now shows the integral above equals

$$
\left(\frac{(-i r)^{\lambda} g(\psi) t^{\lambda+3 / 2}}{N^{\kappa / 4}}\right) \bar{\psi}\left(t_{0}\right) \frac{\delta_{1}^{2 \lambda+1}}{t_{0}^{\lambda+1}} \int_{\Gamma_{0}(4 N) \backslash H} v^{\kappa / 2} G(z) \overline{h_{\psi}\left(t \delta_{1}^{2} z\right)} d_{0} z .
$$

We have arrived at the following theorem:

THEOREM 4.9. Let $N t=m^{2} n^{2} \tau$ be a factorization of $N t$, with $\tau$ squarefree, and let $c=2 m^{2} n \tau c_{2}$ with $\left(c_{2}, n\right)=1$. Suppose $\chi_{t} \bmod 4 N t$ is induced by the primitive character $\psi \bmod r$, and let $Q=\sum_{q=1}^{4 N t} \bar{\chi}_{t}(1+q n)$. Assume $t \mid N$, and $r \mid n$. Then the constant (pole) term of $\Psi_{t}$ at $1 / c$ is

$$
\begin{array}{r}
\left((i \eta)^{\lambda-1} \Psi_{t_{22}^{\prime}}\left(\begin{array}{ll}
1 & 0 \\
c & 1
\end{array}\right)\right)(i \infty)=\frac{(-i)^{\lambda+1} Q \bar{\psi}\left(c_{2}\right) g(\psi)}{4^{\lambda} r} N^{\lambda / 2-3 / 4} t^{(\kappa-2) / 4} \\
\quad \times \sum \mu(d) \psi(d) \bar{\psi}\left(t_{0}\right) \delta^{\lambda} d^{-1} \int_{\Gamma_{0}(4 N) \backslash H} v^{\alpha / 2} G(z) \overline{h_{\psi}\left(t \delta^{2} z\right)} d_{0} z
\end{array}
$$

where the sum is taken over all triples of positive integers $\left(d, \delta, t_{0}\right)$ such that $t_{0} \mid t,\left(t_{0}, \delta\right)=1$, and $n=r t d \delta / t_{0}$. 
Proof. This is a simple calculation based on (4.22) and (4.29).

Corollary 4.10 (Shimura's Conjecture). Let $G \in S_{3 / 2}(4 N, \chi)$. Then $S_{t}(G)$ is a cusp form if and only if $G \perp U_{t}$.

Proof. Recall that $U_{t}$ is spanned by all $h_{\psi}\left(t \delta^{2} z\right)$ such that $\psi$ is a primitive character, mod $r$, inducing $\chi_{t}$, and $t \delta^{2} r^{2} \mid N$. In (4.30) above, note that, if $t \delta^{2} r^{2} \nmid N$, then $\left(t_{0}, r\right)>1$, so that $\psi\left(t_{0}\right)=0$. Thus the only inner products appearing on the RHS of (4.30) are exactly those $h_{\psi}$ 's spanning $U_{t}$.

So $G \perp U_{t}$ implies $S_{t}(G)$ is a cusp form. The converse also holds, by an induction argument identical to the case $t=1$.

Corollary 4.11 (weight $1 / 2$ analogue). Let $G \in G_{1 / 2}^{\prime}(4 N, \chi) . \quad\left(G_{1 / 2}^{\prime}\right.$ means $a(0)=0$ if $\chi$ is principal). Then $S_{t}(G)$ is bounded on $H$ if and only if $G \perp U_{t} . \quad\left(\right.$ Here $\left.U_{t}=\left\{h_{\psi}\left(t d^{2} z\right)\left|4 t d^{2} r^{2}\right| 4 N, \chi=\psi(t /)\right\}.\right)$

\section{§ 5. Examples}

One important property of the Shimura lift $S_{t}$ is that it commutes with the action of Hecke operators. We shall state this precisely.

Let

$$
G(z)=\sum_{n=0}^{\infty} a(n) e(n z) \in G_{\kappa / 2}(4 N, \chi) \quad \text { and } \quad \Phi(w)=\sum_{n=0}^{\infty} A(n) e(n w) \in G_{k}(N, \phi) .
$$

Let $p$ be a prime. Define

$$
T\left(p^{2}\right) G=T_{\kappa, x}^{4 N}\left(p^{2}\right) G=\sum_{n=0}^{\infty} b(n) e(n z) \quad \text { and } \quad T(p) \Phi=T_{k, \phi}^{N}(p) \Phi=\sum_{n=0}^{\infty} B(n) e(n w)
$$

by

$$
\begin{aligned}
& b(n)=\left\{\begin{array}{ll}
a\left(n p^{2}\right) \quad \text { if } p \mid 4 N \\
a\left(n p^{2}\right)+p^{\lambda-1} \chi_{n}(p) a(n)+p^{2 \lambda-1} \chi^{2}(p) a\left(n / p^{2}\right)
\end{array} \text { if } p \nmid 4 N\right. \\
& B(n)= \begin{cases}A(n p) \quad \text { if } p \mid N \\
A(n p)+\phi(p) p^{k-1} A(n / p) & \text { if } p \nmid N .\end{cases}
\end{aligned}
$$

(Here $A(x)=a(x)=0$ if $x \notin Z$.)

Proposition 5.1. $S_{t}\left(T\left(p^{2}\right) G\right)=T(p) S_{t}(G)$, for all primes $p$ and all square-free $t$.

Proof. A straightforward verification based on the result

$$
A_{t}(n)=\sum_{d \mid n} \chi_{t}(d) d^{2-1} a\left(t(n / d)^{2}\right) .
$$

Even more significant is the result of Shimura: 
TheOREM 5.2. Suppose $G=\sum_{n=0}^{\infty} a(n) e(n z) \in G_{\kappa / 2}(4 N, \chi)$ is an eigenform for all Hecke operators $T\left(p^{2}\right)$. Then for any square-free positive integers $t$, $\tau$, we have $a(t) S_{\tau}(G)=a(\tau) S_{t}(G)$.

EXAMPLE 1). The space $G_{3 / 2}(4,1)$ is 1 -dimensional, spanned by

$$
\theta^{3}(z)=\sum_{n=0}^{\infty} r_{3}(n) q^{n}=1+6 q+12 q^{2}+8 q^{3}+6 q^{4}+\cdots
$$

Being the only function in the space, $\theta^{3}$ is necessarily a common Hecke eigenform. The Shimura lifting sends

$$
\theta^{3} \rightarrow A_{1}(0)+6 q+6 q^{2}+\cdots \in G_{2}(2,1) .
$$

The latter is also one-dimensional, spanned by $G_{2}(z, 0,0,2)-G_{2}(z, 0,1,2)$ (see Section 4; alternatively, the lift of $\theta^{3}$ can be identified as (1/4)T(2) $\theta^{4}$.) We find $A_{1}(0)=1 / 4$. Since $r_{3}(1)=6$, Theorem 5.2 gives us, for squarefree $t$,

$$
r_{3}(t)=\frac{a(1) A_{t}(0)}{A_{1}(0)}=24 A_{t}(0)
$$

Now Theorem 2.17 shows, for $\lambda=1$

$$
A_{t}(0)=\frac{a(0)}{2 \pi i} \sum_{m=1}^{\infty} \check{\chi}_{t}(m) / m
$$

where $\chi_{t}=(-t /)$. It is not difficult to prove that

$$
\sum_{m=1}^{\infty} \hat{\chi}_{t}(m) / m^{s}=\left\{\begin{array}{l}
(4 t)^{1 / 2} i \sum_{m=1}^{\infty} \chi_{t}(m) / m^{s} \quad t \equiv 1,2(4) \\
(4 t)^{1 / 2} i\left(2^{1-2 s}-\left(\frac{2}{t}\right) 2^{-s}\right) \sum_{m=1}^{\infty}\left(\frac{m}{t}\right) / m^{s} \quad t \equiv 3(4) .
\end{array}\right.
$$

Putting together the last three equations, we derive the well-known result

$$
r_{3}(t)=\left\{\begin{array}{rlrl}
\frac{24}{\pi}(t)^{1 / 2} \sum_{m=1}^{\infty}\left(\frac{-t}{m}\right) / m & t \equiv 1,2(4) \\
0 & t \equiv 7(8) \quad(t \text { square-free }) \\
\frac{24}{\pi}(t)^{1 / 2} \sum_{m=1}^{\infty}\left(\frac{m}{t}\right) / m & t \equiv 3(8) .
\end{array}\right.
$$

Alternatively, we may use (2.64) and derive $r_{3}(t)=12 L\left(0, \chi_{t}\right)$.

ExAmple 2). Dimensions for spaces of modular forms have been calculated by Cohen and Oesterlé [1]. For weight 3/2, the first cusp forms appear at level 28: 


$$
\operatorname{dim} S_{3 / 2}(28,1)=\operatorname{dim} S_{3 / 2}\left(28,\left(\frac{7}{)}\right)\right)=1
$$

Clearly, there are no theta functions in these spaces. (The first theta function appears in $S_{3 / 2}(36,(3 /))$ ). $)$ Thus, by Corollary 4.10 (Shimura's conjecture), these functions lift to cusp forms:

$$
S_{3 / 2}(28, *) \stackrel{S_{t}}{\longrightarrow} S_{2}(14,1)
$$

On the other hand, multiplication by $\theta(z)$ (resp. $\theta(7 z)$ ) sends $S_{3 / 2}(28,1)$ (resp. $\left.S_{3 / 2}(28,(7 /))\right)$ to $S_{2}(28,1)$ : Now $S_{3}(14,1)$ is 1-dimensional, spanned by

$$
\phi(z)=(\Delta(z) \Delta(2 z) \Delta(7 z) \Delta(14 z))^{1 / 24}=q-q^{2}-2 q^{3}+q^{4}+\cdots
$$

where

$$
\Delta(z)=q \prod_{n=1}^{\infty}\left(1-q^{n}\right)^{24} \quad(q=e(z))
$$

$S_{2}(28,1)$ is 2 -dimensional, spanned by $\phi(z)$ and $\phi(2 z)$.

$$
\begin{aligned}
& \text { Suppose now } G(z)=a(1) q+a(2) q^{2}+\cdots \in S_{3 / 2}(28,1) \text {. } \\
& \text { Then } S_{1}(G)=a(1) q+a(4) q^{2}+\cdots=a(1) \phi(z),
\end{aligned}
$$

and

$$
\begin{aligned}
G(z) \theta(z) & =a(1) q+(a(2)+2 a(1)) q^{2}+(a(3)+2 a(2)) q^{3}+(a(4)+2 a(3)) q^{4} \cdots \\
& =\alpha \phi(z)+\beta \phi(2 z)
\end{aligned}
$$

for some $\alpha$ and $\beta$. We claim first of all that $a(1) \neq 0$. For suppose $a(1)$ $=0$; then $G(z) \theta(z)=\beta \phi(2 z)$, so that $a(2)=\beta, a(3)=-2 \beta$, and $a(4)=3 \beta$, from which we conclude that $S_{1}(G)=3 \beta q^{2}+\cdots, \neq 0$, which contradicts (5.6).

We may therefore assume $a(1)=1$. Then (5.6) shows that $a(4)=-1$. Plugging these values into (5.7) and equating coefficients of $q, q^{2}, q^{3}, q^{4}$, gives four equations in four unknowns $(a(2), a(3), \alpha, \beta)$; the unique solution is $\alpha=1, \beta=2, a(2)=-1, a(3)=0$. Thus

$$
G(z)=\frac{\phi(z)+2 \phi(2 z)}{\theta(z)}=q-q^{2}-q^{4}+\cdots \text { spans } S_{3 / 2}(28,1) .
$$

A similar analysis shows

$$
G(z)=\frac{\phi(z)-2 \phi(2 z)}{\theta(7 z)}=q-3 q^{2}-2 q^{3}-q^{4}+\cdots \text { spans } S_{3 / 2}(28,(\underline{7})) .
$$

Example 3). Our last example is to compute the Petersson inner product 


$$
\int_{\Gamma\left(4 r^{2}\right) \backslash H} v^{\kappa / 2} h_{\psi}(z) \overline{h_{\psi}(z)} d_{0} z
$$

for theta functions of weight $1 / 2$ and $3 / 2$, using Theorems 4.4 and 3.4. We shall need the following result, which we state without proof.

Proposition 5.3. Let $\Psi=\left.\Phi\right|_{2 \lambda} W(2 N)$ be modular forms of weight $2 \lambda$ and level 2N. Then

$$
\left[\left.(i \eta)^{\lambda-1} \Psi\right|_{2 \lambda}\left(\begin{array}{ll}
1 & 0 \\
c & 1
\end{array}\right)\right](i \infty)=\left(\frac{c^{2}}{2 N}\right)\left[\left.(i \eta)^{\lambda-1} \Phi\right|_{2 \lambda}\left(\begin{array}{cc}
1 & 0 \\
-2 N / c & 1
\end{array}\right)\right](i \infty) .
$$

Now let $\Phi(w)=N_{1}\left(h_{\psi}\right)$ be the Niwa lift of $h_{\psi}(z)$. Taking $c=2 r$, Proposition 5.3 above and Theorem 4.4 (5) combine to give

$$
\begin{aligned}
& {\left[\left.(i \eta)^{\lambda-1} N_{1}\left(h_{\psi}\right)\right|_{2 \lambda}\left(\begin{array}{cc}
1 & 0 \\
-r & 1
\end{array}\right)\right](i \infty)} \\
& \quad=\frac{1}{2}\left[\frac{(-i)^{\lambda+1} Q \Psi(1) g(\psi)}{4^{\lambda} r} r^{\lambda-3 / 2} \int_{\Gamma_{0}\left(4 r^{2}\right) \backslash H} v^{\kappa / 2} h_{\psi}(z) \overline{h_{\psi}}(z) d_{0} z\right] \\
& \quad=\left((-i)^{\lambda+1} 2^{1-2 \lambda} r^{\lambda-1 / 2} g(\psi) / \delta\right) \int_{\Gamma_{0}\left(4 r^{2}\right) \backslash H} v^{\kappa / 2} h_{\psi}(z) \overline{h_{\psi}(z)} d_{0} z
\end{aligned}
$$

where

$$
\delta=\left\{\begin{array}{l}
1 \text { if } r \text { is even } \\
2 \text { if } r \text { is odd }
\end{array} \text { (this since } Q=\sum_{q=1}^{4 r^{2}} \psi_{1}(1+q r)=4 r^{2} / \delta\right) .
$$

On the other hand, by Corollary 3.2 and Theorem 3.4,

$$
\begin{aligned}
{\left[\left.(i \eta)^{\lambda-1} S_{1}\left(h_{\psi}\right)\right|_{2 \lambda}\left(\begin{array}{cc}
1 & 0 \\
-r & 1
\end{array}\right)\right](i \infty) } & =\left.(2 \pi i)^{1-\lambda} E\right|_{2}\left(\begin{array}{cc}
1 & 0 \\
-r & 1
\end{array}\right)(i \infty) \\
& =(2 \pi i)^{1-\lambda}\left(\frac{-r \psi(-1) g(\psi)}{24}\right) \prod_{p \mid 4 r^{2}}\left(1-p^{-2}\right) .
\end{aligned}
$$

Finally, by Theorem 2.17 (and Equation (3.3)),

with

$$
\begin{gathered}
N_{1}(G)=C_{1}(\lambda) S_{1}(G) \\
C_{1}(\lambda)=\left\{\begin{array}{cc}
8 r^{1 / 2} & \lambda=0 \\
-1 / 2 r^{3 / 2} & \lambda=1
\end{array}\right\}=(-1)^{\lambda} r^{2+1 / 2} 2^{3-4 \lambda} .
\end{gathered}
$$

(This form for $C_{1}(\lambda)$ holds only for $\lambda=0,1$ !) Putting all this together gives

$$
\int_{\Gamma_{0}\left(4 r^{2}\right) \backslash H} v^{\kappa / 2} h_{\psi}(z) \overline{h_{\psi}(z)} d_{0} z=\left\{\begin{array}{ll}
\frac{\pi \delta r^{2}}{3} \prod_{p \mid 42^{2}}\left(1-p^{-2}\right) & \kappa=1 \\
\frac{\delta r^{2}}{24} \prod_{p \mid 4 r^{2}}\left(1-p^{-2}\right) & \kappa=3
\end{array} .\right.
$$


As a special case, let $\psi(m)=(m / p)$ for an odd prime $p$. Then

$$
\int_{\Gamma_{0\left(4 p^{2}\right) \backslash H}} v^{\kappa / 2} h_{\psi}(z) \overline{h_{\psi}(z)} d_{0} z=\left\{\begin{array}{lll}
\frac{\pi}{2}\left(p^{2}-1\right) & p \equiv 1(4) & (\kappa=1) \\
\frac{p^{2}-1}{24} & p \equiv 3(4) \quad(\kappa=3)
\end{array} .\right.
$$

These formulae are in agreement with the results of Petersson [9]. The derivation of course is different.

\section{Appendix A. Characters and character sums}

We present here some results concerning characters which are used elsewhere in the paper. The results are elementary, but worth writing down.

1) The quadratic residue symbol

We wish to define precisely our use of the "quadratic residue symbol" $(c / d)$. We follow Shimura's definition.

$$
\text { Let } \quad c=\prod_{i=0}^{m} p_{i} \quad \text { and } \quad d=\prod_{j=0}^{n} q_{j}, \quad \text { where } p_{0}, q_{0} \in\{-1,0,1\}
$$

and all other $p_{i}, q_{j}$ are positive primes. Define, multiplicatively,

$$
\left(\frac{c}{d}\right)=\prod_{i=0}^{m} \prod_{j=0}^{n}\left(\frac{p_{i}}{q_{j}}\right)
$$

where

(1) $\left(\frac{p}{q}\right)=0$ if $(p, q)>1$

(2) $\left(\frac{p}{1}\right)=1$

(3) $\left(\frac{p}{-1}\right)=\left\{\begin{aligned} 1 & \text { if } p \geq 0 \\ -1 & \text { if } p<0\end{aligned}\right\}$

(4) if $q \notin\{-1,0,1,2\}$

$$
\left(\frac{p}{q}\right)=\left\{\begin{aligned}
1 & \text { if } x^{2} \equiv p \bmod q \text { has } 2 \text { solutions } \\
-1 & \text { if } x^{2} \equiv p \bmod q \text { has } 0 \text { solutions }
\end{aligned}\right.
$$

(5) $\left(\frac{p}{2 q}\right)=0$ if $p \equiv-1 \bmod 4$

(6) $\left(\frac{p}{2 q}\right)=\left(\frac{p}{p+2 q}\right)$ if $p \equiv 1 \bmod 4$

for $p \in\left\{p_{i} \mid i=0,1, \cdots, m\right\}$ and $q \in\left\{q_{j} \mid j=0,1, \cdots, n\right\}$. 
This definition has the advantage of presenting $(c / d)$ as an obviously well-defined symbol. However, it obscures the character qualities of the object. Let us state what these are:

(1) If $0<d$ is odd, then $\chi(c)=(c / d)$ is a character mod $d$.

(2) If $\tau$ is square-free, then $\chi(d)=(\tau / d)$ is a character mod $t$, where $t=|\tau|$ if $\delta \mid \tau$ implies $\delta \equiv 1 \bmod 4$ $t=4|\tau|$ otherwise

(3) If $\tau$ is the square-free part of $c$, then $\chi(d)=(c / d)$ is the character induced by $(\tau / d)$. That is, $(c / d)=(\tau / d)$ whenever $(d, c)=1$.

Note that in our use of the quadratic residue symbol, $(c / 2)=0$ whenever $c$ contains a prime factor congruent to $-1 \bmod 4$.

2) Primitive and induced characters

A character $\psi \bmod r$ induces the character $\chi \bmod M$ when $r$ is a proper divisor of $M$ and $\psi(m)=\chi(m)$ for $(m, M)=1$. The character $\psi \bmod r$ is primitive if it is induced by no other character. (In this paper, $\psi$ nearly always refers to a primitive character $\bmod r$.)

If $\chi \bmod M$ is induced by $\psi \bmod r$, and $f$ is any function on $Z$, then, formally at least,

$$
\sum_{m=1}^{\infty} \chi(m) f(m)=\sum_{d \mid M} \mu(d) \psi(d) \sum_{m=1}^{\infty} \psi(m) f(d m)
$$

where $\mu$ is the Möbius function. (This formula is generally true- $\psi$ need not be primitive.)

3) A character sum

Proposition A. 1 . Let $\chi$ be a character $\bmod M$, induced by the primitive character $\psi \bmod r$. Let $n \mid M$, and define

$$
S(k)=\sum_{q=1}^{M} \chi(k+q n) .
$$

Then

(1) If $r \nmid n, S(k)=0$ for all $k$;

(2) If $r \mid n, S(k)$ is, up to a nonzero multiplicative constant, the character $\bmod n$ induced by $\psi$. That constant is $Q=\sum_{q=1}^{M} \chi(1+q n)$.

Proof. Since $n \mid M$ and $\chi$ is $\bmod M, S(k)$ is defined modulo $n$, and the sum may be taken over any complete residue system $\bmod M$. Now $(k, n)>1$ implies $\chi(k+q n)=0$ for all $q$, hence $S(k)=0$ for $(k, n)>1$. Assume $(k, n)=1$. Then there is some $k^{\prime}, k^{\prime} \equiv k \bmod n$, with $\left(k^{\prime}, M\right)=1$. 
(By Dirichlet's Theorem, $k^{\prime}$ may be a large prime.) We have

$$
\begin{aligned}
S(k)=S\left(k^{\prime}\right) & =\sum_{q \bmod M} \chi\left(k^{\prime}+q n\right)=\sum_{q \bmod M} \chi\left(k^{\prime}+k^{\prime} q n\right) \\
& =\chi\left(k^{\prime}\right) \sum_{q=1}^{M} \chi(1+q n) .
\end{aligned}
$$

When $r \mid n, \chi(1+q n)$ takes only the two values 1 and 0 , and is 1 at least once (at $q=0$ ), so $Q=\sum \chi(1+q n) \neq 0$, establishing (2). Conversely, if $Q \neq 0$, then up to this nonzero multiplicative constant, $S(k)$ is a character $\bmod n$, inducing $\chi$ and therefore induced by $\psi$. Thus $r \mid n$, establishing (1).

\section{Appendix B. Error Estimates}

We wish to present in detail the estimates showing that only $\hat{x}_{3}=0$ contributes in the limit $\eta \rightarrow \infty$ in Section 4 and that the other errors incurred in that section also are negligible.

We first show that the integral over $\Gamma_{0}(4 N) \backslash H$ may be approximated by an integral over a certain compact region.

Proposition B. 1. Let $\mathscr{F}=\{z|| z|>1,| \operatorname{Re} z \mid<1 / 2\}$ be the usual fundamental domain for $S L(2, Z)$, and let $\mathscr{F}_{\eta}=\mathscr{F} \cap\left\{z \mid \operatorname{Im} z<\eta^{2}\right\}$. Let

$$
\Gamma_{0}(4 N) \backslash H \simeq D=\bigcup_{r \in S L(2, Z) \backslash \Gamma_{0}(4 N)} \gamma \mathscr{F}
$$

and let

$$
D_{\eta}=\bigcup_{\gamma} \gamma_{F_{\eta}} \cdot
$$

Suppose $F \in G_{\kappa / 2}(4 N, \bar{\chi}(N /))$ (with $F \mid W(4 N)(i \infty)=0$ if $\kappa=1$ and $\chi=1$ ). Then

$$
\int_{D} v^{\kappa / 2} F(z) \overline{\theta_{\gamma}(z, i \eta)} d_{0} z=\int_{D_{\eta}} v^{k / 2} F(z) \overline{\theta_{\gamma}(\bar{z}, \overline{i \eta})} d_{0} z+\left\{\begin{array}{ll}
O(1) & \text { if } F \notin S_{\kappa / 2}(4 N, \bar{\chi}(N /)) \\
o(1) & \text { if } F \in S_{\kappa / 2}(4 N, \bar{\chi}(N /))
\end{array} .\right.
$$

Proof. See the proof of Proposition 2.8. Generally, if $F \in P_{k / 2}^{m}$ then the error estimate is $O\left(\eta^{2 m}\right)$.

Remark. The error $O(1)$ for $F \in G_{1 / 2}$ becomes an error $o(1)$ when we divide by $\eta$ to get the pole term.

Proposition B. 2. Consider the decomposition

$$
\theta_{r}(z, i \eta)=\sum_{\hat{x}_{3}=0}+\sum_{\hat{x}_{3} \neq 0} .
$$


(Equation (4.4) makes clear the notation on the right.) Then

$$
\sum_{\hat{x}_{3} \neq 0}=O\left(e^{-\alpha v \eta^{2}} \eta v^{-1 / 2}\right) \quad v \in D_{\eta}
$$

for some positive constant $\alpha$.

Proof. Observe the general estimate

$$
\begin{aligned}
\left|\theta_{r}(z, i \eta)\right| \leq & \sum \frac{v^{1 / 2}}{\eta^{2}}|\Lambda(\hat{x}, i \eta)|^{\mid} \exp \left[-\frac{4 \pi v}{N}\left(\frac{1}{16 \eta^{2}} \hat{x}_{1}^{2}+16 \eta^{2} \hat{x}_{3}^{2}\right)\right] \\
& \times \exp \left[-\frac{2 \pi v}{N} \hat{x}_{2}^{2}\right] .
\end{aligned}
$$

Now

$$
\begin{aligned}
|\Lambda(\hat{x}, i \eta)| & =\left|\left(\frac{1}{4 \eta} \hat{x}_{1}-4 \eta \hat{x}_{3}\right)-i \hat{x}_{2}\right| \\
& \leq \frac{1}{4 \eta}\left|\hat{x}_{1}\right|+4 \eta\left|\hat{x}_{3}\right|+\left|\hat{x}_{2}\right| .
\end{aligned}
$$

When $\hat{x}_{3} \neq 0$, we have

$$
|\Lambda(\hat{x}, i \eta)| \leq C \eta\left|\hat{x}_{3}\right|\left(1+\frac{1}{4 \eta}\left|\hat{x}_{2}\right|+\frac{1}{16 \eta^{2}}\left|\hat{x}_{1}\right|\right)
$$

where $C$ depends only on the lattice $\hat{\gamma} L$ '. For "large" $\eta$ (such as $\eta>1 / 4$ ), we have

$$
|\Lambda|^{\lambda} \leq C_{1} \eta^{\lambda}\left|\hat{x}_{3}\right|^{\lambda}\left(1+\eta^{-\lambda}\left|\hat{x}_{2}\right|^{\lambda}+\eta^{-2 \lambda}\left|\hat{x}_{1}\right|^{\lambda}\right) .
$$

From (B. 3) and (B. 4), we get the estimate

$$
\sum_{\hat{x} 3 \neq 0} \leq C_{1} v^{1 / 2} \sum_{\hat{x_{3}} 3}\left[\sum_{\hat{x}_{1}} 0 \sum_{\hat{x}_{2}}+\eta^{-\lambda} \sum_{\hat{x}_{1}} 0 \sum_{\hat{x}_{2}} \lambda+\eta^{-2 \lambda} \sum_{\hat{x}_{1}} x_{-} \sum_{\hat{x}_{2}}\right]
$$

where

$$
\begin{aligned}
\sum_{\hat{x}_{3}} \lambda & =\sum_{\hat{x}_{3} \neq 0}\left|\hat{x}_{3}\right|^{\lambda} \exp \left(-\frac{64 \pi v \eta^{2}}{N} \hat{x}_{3}^{2}\right) \\
& =O\left(e^{-\alpha v \eta^{2}}\left(1+\left(v \eta^{2}\right)^{-(\lambda-1) / 2}\right)\right) \\
& =O\left(e^{-\alpha^{\prime} v \eta^{2}}\right) \quad v \in D_{\eta} .
\end{aligned}
$$

(One may take $\alpha$ to be $64 \pi / N$ times the smallest value of $\hat{x}_{3}^{2}$, and $\alpha^{\prime}$ to be half of $\alpha$.)

$$
\sum_{\hat{x}_{1}}=\sum_{\hat{x} 1}\left|\hat{x}_{1}\right|^{\nu} \exp \left(-\frac{\pi v}{4 N \eta^{2}} \hat{x}_{1}^{2}\right)=O\left(1+\left(v / \eta^{2}\right)^{-(\nu+1) / 2}\right)
$$




$$
\sum_{\hat{x}_{2}}=\sum_{\hat{x}_{2}}\left|\hat{x}_{2}\right|^{\nu} \exp \left(-\frac{\pi v}{4} \hat{x}_{2}^{2}\right)=O\left(1+v^{-(\nu+1) / 2}\right) .
$$

Combining (B. 6, 7, 8) in (B. 5) gives an estimate

$$
\begin{aligned}
\sum_{\hat{x} \rightarrow \neq 0} & =O\left[e^{-\alpha^{\prime \prime} v \eta^{2}} v^{1 / 2}\left(\left(v / \eta^{2}\right)^{-1 / 2} v^{-1 / 2}+\eta^{-\lambda}\left(v / \eta^{9}\right)^{-1 / 2} v^{-(\lambda+1) / 2}+\eta^{-2 \lambda}\left(v / \eta^{2}\right)^{-(\lambda+1) / 2} v^{-1 / 2}\right)\right] \\
& =O\left[e^{-\alpha^{\prime \prime} v \eta^{2}}\left(\eta v^{-1 / 2}+\eta^{1-\lambda} v^{-(\lambda+1) / 2}+\eta^{1-\lambda} v^{-(\lambda+1) / 2}\right)\right] \\
& =O\left[e^{-\alpha^{\prime \prime} v \eta \eta^{2}} \eta v^{-1 / 2}\left(1+\left(v \eta^{2}\right)^{-\lambda / 2}\right)\right] \\
& =O\left[e^{-\alpha^{\prime \prime \prime} v \eta^{2}} \eta v^{-1 / 2}\right] \quad \text { for } v \in D_{\eta} .
\end{aligned}
$$

Proposition B. 3.

$$
\int_{D_{\eta}} v^{\kappa / 2} F(z) \sum_{\hat{x}_{s} \neq 0} d_{0} z= \begin{cases}o(1) & \text { if } F \in S_{\kappa / 2}\left(4 N, \bar{\chi}\left(\frac{N}{-}\right)\right) \\ o(\eta) & \text { if } F \in G_{1 / 2}\left(4 N, \bar{\chi}\left(\frac{N}{)}\right)\right)\end{cases}
$$

Proof. We use the estimate (B. 2). For cusp forms the result is obvious: small values of $v$ may be ignored, and the exponential factor $e^{-\alpha v \eta^{2}}$ takes care of the rest. In the second case, we must concern ourselves with small values of $v$, say in the range $\eta^{-1}>v>\eta^{-2}$. In this range $\sum_{\hat{x}_{3} \neq 0}=O\left(\eta v^{-1 / 2}\right)$. But since $O\left(v^{-1 / 2}\right)$ is the estimate for modular forms of weight $1 / 2$, we have

$$
\int_{D} v^{\kappa / 2} F(z) O\left(v^{-1 / 2}\right) d_{0} z=O(1)
$$

Therefore, the integral over a vanishingly small piece of $D$ will be $o(1)$. The inclusion of $\eta$ into the estimate $O\left(v^{-1 / 2}\right)$ gives the desired result.

Remark. Again, the lower estimate in (B. 9) becomes o(1) upon division by $\eta$.

Proposition B. 4. The discrepancy between (4.10) and (4.11) is $O\left(\eta^{-1}\right)$, and

$$
\eta^{-1} \int_{D_{\eta}} v^{\kappa / 2}|F(z)| d_{0} z=o(1)
$$

Proof. The derivation between (4.10) and (4.11) notes an error $O\left(v^{1 / 2} / \eta\right)$. This occurs within the theta function (4.10), which is easily seen to have no constant term. (When $k=0, \bar{\chi}_{1}(h)=0$ ). Without the expression in parentheses, (4. 10) has the estimates $O\left(e^{-\alpha v}\left(1+v^{-1 / 2}\right)\right)$, for some positive $\alpha$; with the estimate $O\left(v^{1 / 2} / \eta\right)$ we get an overall estimate $O\left(\eta^{-1} e^{-\alpha v}\left(v^{1 / 2}+1\right)\right)$. This 
clearly weakens to $O\left(\eta^{-1}\right)$. As for the estimate (B. 12), it holds since the integral is absolutely convergent even when $D_{\eta}$ is replaced by $D$.

Proposition B. 5. Let $H_{\lambda}(z)$ be as in (4.14). Then

$$
\int_{D} v^{\kappa / 2} F(z) H_{\lambda}\left(m^{2} \tau z\right) d_{0} z=\int_{D_{\eta}} v^{\kappa / 2} F(z) \overline{H_{\lambda}\left(m^{2} \tau z\right)} d_{0} z+o(1) .
$$

Proof. The integral at left is absolutely convergent.

\section{REFERENCES}

[1] H. Cohen and J. Oesterlé, Dimensions des espaces de formes modulaires, Modular Functions of One Variable VI, Lecture Notes in Math., Springer-Verlag, 627 (1977), 69-78.

[2] Y. Flicker, Automorphic forms on covering groups of $G L(2)$, Invent. Math., 57 (1980), 119-182.

[ 3 ] S. Gelbart and I. Piatetski-Shapiro, On Shimura's Correspondence for Modular Forms of Half-Integral Weight, Proc., Colloquium on Automorphic Forms, Representation Theory and Arithmetic, Bombay, 1979.

[4] E. Hecke, Analytische Arithmetik der Positiven Quadratischen Formen, Kgl. Danske Videnskabernes Selskab. Mathematisk-Fysiske Meddelelser, XVII, 12 (1940), 134S, (= Werke, 789-918).

[5] — Theorie der Eisensteinschen Reihen Höherer Stufe und Ihre Anwendung auf Funktiontheorie und Arithmetik, Abhand. Math. Seminar Hamburg, 5 (1927), 199-224, (= Werke, 461-486).

[ 6 ] J. Igusa, Theta Functions, Springer-Verlag, 1972.

[ 7 ] H. Kojima, Cusp forms of weight 3/2, Nagoya Math. J., 79 (1980), 111-122.

[ 8 ] S. Niwa, Modular forms of half integral weight and the integral of certain thetafunctions, Nagoya Math. J., 56 (1974), 147-161.

[9] H. Petersson, UUber die Berechnung der Skalarprodukte ganzer Modulformen, Comment. Math. Helv., 22 (1949), 168-199.

[10] J.-P. Serre and H. Stark, Modular forms of weight 1/2, Modular Functions of One Variable VI, Lecture Notes in Math., Springer-Verlag, 627 (1977), 29-67.

[11] G. Shimura, On modular forms of half integral weight, Ann. of Math., 97 (1973), $440-481$.

[12] T. Shintani, On construction of holomorphic cusp forms of half integral weight, Nagoya Math. J., 58 (1975), 83-126.

[13] H. Cohen, A lifting of modular forms in one variable to Hilbert modular forms in two variables, Modular Functions of One Variable VI, Lecture Notes in Math., Springer-Verlag, 627 (1977), 175-196.

Department of Mathematics

Ohio State University

Columbus, Ohio 43210

USA 